\title{
Supergravity-matter actions in three dimensions and Chern-Simons terms
}

\author{
Sergei M. Kuzenko and Joseph Novak \\ School of Physics M013, The University of Western Australia, \\ 35 Stirling Highway, Crawley W.A. 6009, Australia \\ E-mail: sergei.kuzenko@uwa.edu.au, joseph.novak@uwa.edu.au
}

Abstract: We study off-shell $\mathcal{N}$-extended Yang-Mills multiplets coupled to conformal supergravity in three spacetime dimensions. Superform formulations are presented for the non-Abelian Chern-Simons actions in the cases $\mathcal{N}=1,2,3$, and the corresponding component actions are explicitly worked out. Such a Chern-Simons action does not exist for $\mathcal{N}=4$. In the latter case, a superform formulation is given for the $B F$ term that describes the coupling of two Abelian vector multiplets with self-dual and anti-self-dual superfield strengths respectively. The superform results obtained are used to construct linear multiplet action principles in the cases $\mathcal{N}=2,3,4$. The $\mathcal{N}=3$ and $\mathcal{N}=4$ actions are demonstrated to be universal in the sense that all known off-shell supergravity-matter systems (with the exception of pure conformal supergravity) may be described using such an action. Starting from the $\mathcal{N}=3$ and $\mathcal{N}=4$ Abelian vector multiplets, we also construct composite $\mathcal{O}(2)$ multiplets which are analogues of the four-dimensional construction of an $\mathcal{N}=2$ reduced chiral scalar engineered from the improved tensor multiplet. Using these composites, we present the superfield equations of motion for $\mathcal{N}=3$ and $\mathcal{N}=4$ anti-de Sitter and topologically massive supergravity theories. We also sketch the construction of a large family of higher derivative couplings for $\mathcal{N}=3$ and $\mathcal{N}=4$ vector multiplets.

Keywords: Extended Supersymmetry, Superspaces, Chern-Simons Theories, Supergravity Models

ARXIV EPRINT: 1401.2307

Dedicated to Professor Martin Roček on the occasion of his 60th birthday 


\section{Contents}

1 Introduction 1

2 Vector multiplets in conformal superspace 3

2.1 The $\mathcal{N}=1$ case 4

2.2 The $\mathcal{N}>1$ case 4

3 Chern-Simons and curvature induced three-forms 5

4 Non-Abelian curvature induced three-form $\quad 7$

4.1 The $\mathcal{N}=1$ case 8

4.2 The $\mathcal{N}=2$ case 8

4.3 The $\mathcal{N}=3$ case 9

$\begin{array}{ll}4.4 \text { The special case of } \mathcal{N}=4 & 10\end{array}$

5 Component actions $\quad \mathbf{1 2}$

5.1 Vector multiplets in components 13

$\begin{array}{ll}5.2 \text { Off-shell component actions } & 15\end{array}$

5.2.1 The non-Abelian $\mathcal{N}=1$ case $\quad 15$

5.2.2 The non-Abelian $\mathcal{N}=2$ case $\quad 15$

5.2.3 The non-Abelian $\mathcal{N}=3$ case 16

$\begin{array}{ll}\text { 5.2.4 The Abelian } \mathcal{N}=4 \text { case } & 17\end{array}$

6 Matter-coupled $\mathcal{N}=2$ supergravity $\quad 17$

7 Matter-coupled $\mathcal{N}=3$ supergravity $\quad 20$

$\begin{array}{ll}7.1 \text { Linear multiplet action } & 21\end{array}$

$\begin{array}{lll}7.2 & \text { Composite } \mathcal{O}(2) \text { multiplet } & 21\end{array}$

$\begin{array}{lll}7.3 & \text { Supercurrent } & 22\end{array}$

$7.4(2,1)$ anti-de Sitter supergravity 23

$\begin{array}{ll}7.5 & \text { Topologically massive supergravity }\end{array}$

8 Matter-coupled $\mathcal{N}=4$ supergravity $\quad 27$

$\begin{array}{ll}8.1 \text { Left linear multiplet action } & 27\end{array}$

$\begin{array}{ll}8.2 \text { Right linear multiplet action } & 28\end{array}$

$\begin{array}{ll}8.3 \text { Composite } \mathcal{O}(2) \text { multiplets } & 29\end{array}$

8.4 Supercurrent 31

$8.5(2,2)$ anti-de Sitter supergravity 32

8.6 Topologically massive supergravity 34

9 Concluding comments $\quad 36$ 
A.1 The $\mathcal{N}=1$ case $\quad 39$

$\begin{array}{ll}\text { A.2 } & \text { The } \mathcal{N}=2 \text { case }\end{array}$

$\begin{array}{ll}\text { A.3 The } \mathcal{N}=3 \text { case } & 40\end{array}$

A.4 The $\mathcal{N}>3$ case $\quad 41$

A.5 The $\mathcal{N}=4$ case 42

B Supersymmetry transformations $\quad 42$

C Action principle in $\mathcal{N}=3$ supergravity 44

$\begin{array}{lll}\mathrm{D} & \text { Action principle in } \mathcal{N}=4 \text { supergravity } & 47\end{array}$

\section{Introduction}

The linear multiplet ${ }^{1}$ plays an important role in the context of matter-coupled supergravity theories with eight supercharges in four, five and six dimensions. There are two reasons for the significance of this representation of supersymmetry that can be attributed to its possible realizations as: (i) a dynamical multiplet; or (ii) a composite multiplet. In the first realization, the linear multiplet without central charge [22] (nowadays, often called the $\mathcal{O}(2)$ multiplet $[23,24])$ provides a dual off-shell formulation for the massless hypermultiplet, in which one of the four physical scalars of the hypermultiplet is dualized into a gauge $(d-2)$-form in $d$ dimensions. In the $d=4$ case, the $\mathcal{O}(2)$ multiplet describes the field strength of the massless $\mathcal{N}=2$ tensor multiplet $[8,25]$. In the second realization, which is most relevant for this paper, the linear multiplet takes on the role of a Lagrangian for a locally supersymmetric action $[4,5]$. This action principle turns out to be universal in the sense that it supports general off-shell supergravity-matter theories. ${ }^{2}$ Different

\footnotetext{
${ }^{1}$ In four-dimensional $\mathcal{N}=2$ Poincaré supersymmetry, the linear multiplet was introduced by Sohnius [1] as a superfield Lagrangian for the matter hypermultiplet [2] coupled to the Yang-Mills vector multiplet [3]. The linear multiplet action was generalized to $\mathcal{N}=2$ supergravity by Breitenlohner and Sohnius [4], and then reformulated by de Wit, van Holten and Van Proeyen [5] within the $\mathcal{N}=2$ superconfomal tensor calculus [6-8], see [9] for a recent review. The linear multiplet actions, and their use, in five-dimensional $\mathcal{N}=1$ and six-dimensional $\mathcal{N}=(1,0)$ supergravity theories were described in [10-17] and [18] respectively. It should be mentioned that in five dimensions different authors use different notations, $\mathcal{N}=1$ or $\mathcal{N}=2$, for supersymmetric theories with eight supercharges. The notation $\mathcal{N}=1$ is used, e.g., in refs. [19-21]. The rationale for its use is that the case of eight supercharges corresponds to simple supersymmetry. The alternative notation $\mathcal{N}=2$ is used in [10-17]. The rationale for this choice is that dimensional reduction of five-dimensional theories with eight supercharges leads to $\mathcal{N}=2$ theories in four dimensions. Here we follow the conventions of [19-21].

${ }^{2}$ Its universality may be readily justified in the case of $4 \mathrm{D} \mathcal{N}=2$ supergravity. Within the off-shell formulation for supergravity-matter systems given in [26-28], any dynamical system can be described using the curved projective superspace action. This action can be recast as a chiral action with specially chosen Lagrangian [29]. The latter may equivalently be rewritten, using a simple transformation, as a linear multiplet action.
} 
theories correspond to different composite linear multiplets. In this paper we present three-dimensional (3D) analogues of the linear multiplet action.

The linear multiplet action actually involves two building blocks: an Abelian vector multiplet and a linear multiplet, the latter with or without central charge (no central charge is possible in six dimensions). The vector multiplet is dynamical and model-independent. The linear multiplet is composite and contains all the information about the dynamical system under consideration. Within the superconformal tensor calculus, the action is formulated in terms of the component fields [5], which is useful for many applications. However, this component approach obscures a geometric origin of the action. On the other hand, the action acquires a simple geometric interpretation as a supersymmetric $B F$ term when formulated in curved $4 \mathrm{D} \mathcal{N}=2$ harmonic superspace [30] (as an extension of the rigid supersymmetric construction given in [31]) or, in the case of the linear multiplet without central charge, in curved $4 \mathrm{D} \mathcal{N}=2,5 \mathrm{D} \mathcal{N}=1$ and $6 \mathrm{D} \mathcal{N}=(1,0)$ projective superspaces [19-21, 26-28, 32]. ${ }^{3}$ From the viewpoint of $x$-space practitioners, a disadvantage of these superspace approaches is that some work is required in order to reduce the action to components. Recently, there has appeared a new formulation for the 4D $\mathcal{N}=2$ linear multiplet action [38] that combines the advantages of both the superconformal tensor calculus and the powerful superspace techniques. It made use of $4 \mathrm{D} \mathcal{N}=2$ conformal superspace [39] in conjunction with the superform approach to the construction of supersymmetric invariants [40-43].

The superform formulation given in [38], and its extension to describe $3 \mathrm{D} \mathcal{N}=1$ conformal supergravity [44], has recently been applied to derive off-shell $\mathcal{N}$-extended conformal supergravity actions in three dimensions for the cases $\mathcal{N} \leq 6[45,46] .{ }^{4}$ In the past, the off-shell actions were known only for $\mathcal{N}=1$ [48] (see also [44]) and $\mathcal{N}=2$ [49] conformal supergravities. Refs. [45, 46] made use of the novel off-shell formulation for 3D $\mathcal{N}$-extended conformal supergravity [50] called conformal superspace. ${ }^{5}$ Within the superspace setting of [50], conformal supergravity is simply a gauge theory of the $\mathcal{N}$-extended superconformal group. Conceptually, this supergravity formulation is very similar to that for $\mathcal{N}$-extended Yang-Mills multiplets in superspace. Here we use this analogy to develop a superform realization, in conformal superspace, for $\mathcal{N}$-extended supersymmetric Chern-Simons actions, with $1 \leq \mathcal{N} \leq 4$. Using different techniques, the supersymmetric Chern-Simons actions were originally constructed in $[53,54]$ for the case $\mathcal{N}=1$, in $[55,56]$ for $\mathcal{N}=2,{ }^{6}$ in [57] for $\mathcal{N}=3$. The $\mathcal{N}=4$ supersymmetric $B F$ term was first constructed in components [58], then in $\mathcal{N}=2$ superspace [59], $\mathcal{N}=4$ harmonic superspace [60,61] and also in $\mathcal{N}=3$ harmonic superspace [62]. The $\mathcal{N}=4$ case is actually very special, since a non-Abelian $\mathcal{N}=4$ Chern-Simons action does not exist. This will be discussed in more detail in the main body of our paper.

\footnotetext{
${ }^{3}$ The harmonic $[33,34]$ and projective [35-37] superspaces are powerful approaches to engineer off-shell supersymmetric theories with eight supercharges.

${ }^{4}$ The off-shell action for $3 \mathrm{D} \mathcal{N}=6$ conformal supergravity was independently constructed in [47].

${ }^{5}$ The conventional off-shell formulation for $3 \mathrm{D} \mathcal{N}$-extended conformal supergravity [51, 52], also known as $\mathrm{SO}(\mathcal{N})$ superspace, is obtained from conformal superspace by gauge fixing some of the local symmetries, see [50] for more details. Within the $\mathrm{SO}(\mathcal{N})$ superspace setting, the most general off-shell supergravitymatter couplings were constructed in [52] for the cases $1 \leq \mathcal{N} \leq 4$.

${ }^{6}$ The Abelian $\mathcal{N}=2$ Chern-Simons action was first constructed by Siegel [53].
} 
Using the superform realization of the Chern-Simons actions given, it becomes trivial to construct linear multiplet actions for the cases $\mathcal{N}=2,3,4$; the relevant constructions are given in the main body of our paper. We demonstrate that these actions are actually universal for $\mathcal{N}=3$ and $\mathcal{N}=4$ in the sense that the most general off-shell $\mathcal{N}=3$ and $\mathcal{N}=4$ supergravity-matter systems presented in [52] may be described using the appropriate linear multiplet action. This simplifies the problem of constructing component actions for $\mathcal{N}=3$ and $\mathcal{N}=4$ off-shell supergravity-matter systems. We should emphasize that our statement of universality concerns the off-shell locally supersymmetric theories. The on-shell locally supersymmetric nonlinear sigma models in three dimensions have been described, e.g., in [63-67].

This paper is organized as follows. In section 2 we describe the $\mathcal{N}$-extended nonAbelian vector multiplet in conformal superspace. In section 3 our method to construct supersymmetric Chern-Simons actions is briefly described. In section 4 we derive the curvature induced three-forms for $\mathcal{N} \leq 4$. The component expressions for the supersymmetric Chern-Simons actions with $\mathcal{N} \leq 4$ are given in section 5 . Section 6 is devoted to the $\mathcal{N}=2$ linear multiplet action. In section 7 we work out the $\mathcal{N}=3$ linear multiplet action and apply this construction to the cases of $(2,1)$ anti-de Sitter supergravity and $\mathcal{N}=3$ topologically massive supergravity. In section 8 we work out two $\mathcal{N}=4$ linear multiplet actions and make use of these actions to study $(2,2)$ anti-de Sitter supergravity and $\mathcal{N}=4$ topologically massive supergravity. Some implications of our results and open problems are briefly discussed in section 9 .

We have included a couple of technical appendices. Appendix A includes some salient facts about the conformal superspace of [50]. In appendix B we give the supersymmetry transformations for vector multiplets with $\mathcal{N} \leq 4$. In appendix $\mathrm{C}$ we briefly review covariant projective $\mathcal{N}=3$ supermultiplets and demonstrate universality of the $\mathcal{N}=3$ linear multiplet action. In appendix $\mathrm{D}$ we sketch the structure of left and right covariant projective $\mathcal{N}=4$ supermultiplets and demonstrate universality of the two $\mathcal{N}=4$ linear multiplet actions.

\section{Vector multiplets in conformal superspace}

In this section we show how to describe Yang-Mills multiplets within the superspace formulation of [50], known as conformal superspace. Conformal superspace is based on gauging the entire superconformal algebra. Its essential aspects are summarized in appendix A.

To describe a Yang-Mills multiplet in the $3 \mathrm{D} \mathcal{N}$-extended conformal superspace $\mathcal{M}^{3 \mid 2 \mathcal{N}}$ of [50], parametrized by coordinates $z^{M}=\left(x^{m}, \theta_{I}^{\mu}\right)$, we introduce gauge covariant derivatives

$$
\boldsymbol{\nabla}=E^{A} \nabla_{A}, \quad \nabla_{A}:=\nabla_{A}-\mathrm{i} V_{A},
$$

with $E_{A}=E_{A}{ }^{M} \partial_{M}$ the inverse vielbein, $\nabla_{A}$ the superspace covariant derivatives obeying the (anti-)commutation relations (A.4) and $V=E^{A} V_{A}$ the gauge connection taking its values in the Lie algebra of the Yang-Mills gauge group $G_{\mathrm{YM}}$. The generators of $G_{\mathrm{YM}}$ 
commutes with all the generators of the superconformal algebra (A.3). The Yang-Mills gauge transformation acts on the gauge covariant derivatives as

$$
\nabla_{A} \rightarrow e^{\mathrm{i} \tau} \nabla_{A} e^{-\mathrm{i} \tau}, \quad \tau^{\dagger}=\tau,
$$

where the gauge parameter $\tau(z)$ takes its values in the Lie algebra of $G_{\mathrm{YM}}$.

The gauge covariant derivative algebra is

$$
\begin{aligned}
{\left[\nabla_{A}, \nabla_{B}\right\}=} & -T_{A B}{ }^{C} \nabla_{C}-\frac{1}{2} R(M)_{A B}{ }^{c d} M_{c d}-\frac{1}{2} R(N)_{A B}{ }^{P Q} N_{P Q}-R(\mathbb{D})_{A B} \mathbb{D} \\
& -R(S)_{A B}{ }_{I}^{\gamma} S_{\gamma}^{I}-R(K)_{A B}{ }^{c} K_{c}-\mathrm{i} F_{A B},
\end{aligned}
$$

where the torsion and curvatures are those of conformal superspace but with $F_{A B}$ corresponding to the gauge covariant field strength $F=\frac{1}{2} E^{B} \wedge E^{A} F_{A B}$. The field strength $F_{A B}$ satisfies the Bianchi identity

$$
\nabla F=0, \quad \nabla_{[A} F_{B C\}}+T_{[A B}{ }^{D} F_{|D| C\}}=0
$$

and must be subject to covariant constraints to describe an irreducible vector multiplet. The structure of the constraints and their consequence is different for $\mathcal{N}=1$ and for $\mathcal{N}>1$. Below we describe the various cases.

\subsection{The $\mathcal{N}=1$ case}

In the $\mathcal{N}=1$ case, one imposes the covariant constraint $[53,54]$

$$
F_{\alpha \beta}=0 .
$$

Then one derives from the Bianchi identities the remaining components

$$
\begin{aligned}
F_{a \beta} & =\frac{1}{2}\left(\gamma_{a}\right)_{\beta}^{\gamma} G_{\gamma}, \\
F_{a b} & =-\frac{\mathrm{i}}{4} \varepsilon_{a b c}\left(\gamma^{c}\right)^{\gamma \delta} \nabla_{\gamma} G_{\delta},
\end{aligned}
$$

together with the dimension-2 differential constraint on the spinor field strength

$$
\nabla^{\alpha} G_{\alpha}=0
$$

Furthermore, the Jacobi identities require $G_{\alpha}$ to be primary and of dimension-3/2:

$$
S_{\beta} G_{\alpha}=0, \quad K_{b} G_{\alpha}=0, \quad \mathbb{D} G_{\alpha}=\frac{3}{2} G_{\alpha} .
$$

\subsection{The $\mathcal{N}>1$ case}

For $\mathcal{N}>1$ one imposes the following dimension- 1 covariant constraint $[55,57,68]$

$$
F_{\alpha \beta}^{I J}=-2 \mathrm{i} \varepsilon_{\alpha \beta} G^{I J},
$$

where $G^{I J}$ is antisymmetric, primary and of dimension-1

$$
G^{I J}=-G^{J I}, \quad S_{\alpha}^{I} G^{J K}=0, \quad K_{a} G^{I J}=0, \quad \mathbb{D} G^{I J}=G^{I J} .
$$


These constraints are a natural generalization of the $\mathcal{N}>1$ constraints in four dimensions $[3,69]$. The Bianchi identities then give the remaining field strength components:

$$
\begin{aligned}
F_{a_{\alpha}}^{I} & =\frac{1}{(\mathcal{N}-1)}\left(\gamma_{a}\right)_{\alpha}{ }^{\beta} \nabla_{\beta J} G^{I J}, \\
F_{a b} & =-\frac{\mathrm{i}}{4 \mathcal{N}(\mathcal{N}-1)} \varepsilon_{a b c}\left(\gamma^{c}\right)^{\alpha \beta}\left[\nabla_{\alpha}^{K}, \nabla_{\beta}^{L}\right] G_{K L} .
\end{aligned}
$$

The $\mathcal{N}=2$ case is special because $G^{I J}$ becomes proportional to the antisymmetric tensor $\varepsilon^{I J}$

$$
G^{I J}=\varepsilon^{I J} G
$$

The components of $F_{A B}$ then become

$$
\begin{aligned}
F_{\alpha \beta}^{I J} & =-2 \mathrm{i} \varepsilon_{\alpha \beta} \varepsilon^{I J} G, \\
F_{a \beta}^{J} & =\varepsilon^{J K}\left(\gamma_{a}\right)_{\beta}^{\gamma} \nabla_{\gamma K} G, \\
F_{a b} & =-\frac{\mathrm{i}}{4} \varepsilon_{a b c}\left(\gamma^{c}\right)^{\gamma \delta} \varepsilon^{K L} \nabla_{\gamma K} \nabla_{\delta L} G .
\end{aligned}
$$

The Bianchi identities imply a constraint on $G$ at dimension-2

$$
\boldsymbol{\nabla}^{\gamma I} \nabla_{\gamma}^{J} G=\frac{1}{2} \delta^{I J} \nabla_{K}^{\gamma} \nabla_{\gamma}^{K} G .
$$

Unlike for $\mathcal{N}=2$, in the case $\mathcal{N}>2$ the field strength $G^{I J}$ is constrained by the dimension-3/2 Bianchi identity

$$
\nabla_{\gamma}^{I} G^{J K}=\nabla_{\gamma}^{[I} G^{J K]}-\frac{2}{\mathcal{N}-1} \delta^{I[J} \nabla_{\gamma L} G^{K] L}
$$

This constraint may be shown to define an off-shell supermultiplet, see e.g. [50, 70].

\section{Chern-Simons and curvature induced three-forms}

In this section our method to construct supersymmetric Chern-Simons actions is outlined. This method heavily builds on the superform formalism for the construction of supersymmetric invariants [40-43]. First of all, we sketch its salient points in the framework of 3D $\mathcal{N}$-extended conformal superspace. The formalism makes use of a closed three-form

$$
\mathfrak{J}=\frac{1}{3 !} E^{C} \wedge E^{B} \wedge E^{A} \mathfrak{J}_{A B C}, \quad \mathrm{~d} \mathfrak{J}=0 .
$$

Under an infinitesimal coordinate transformation generated by a vector field $\xi=\xi^{M} \partial_{M}=$ $\xi^{A} E_{A}$, the three-form varies as

$$
\delta_{\xi} \mathfrak{J}=\mathcal{L}_{\xi} \mathfrak{J} \equiv i_{\xi} \mathrm{d} \mathfrak{J}+\mathrm{d} i_{\xi} \mathfrak{J}=\mathrm{d} i_{\xi} \mathfrak{J} .
$$

We note that $\delta_{\xi} \mathfrak{J}=\delta_{\text {gct }} \mathfrak{J}$, where $\delta_{\text {gct }}$ stands for the general coordinate transformation associated with $\xi$. As discussed in appendix A, the gauge group of conformal supergravity, 
$\mathcal{G}$, is generated by two types of transformations: (i) covariant general coordinate transformations, $\delta_{\text {cgct }}$, associated with a parameter $\xi^{A}$; and (ii) standard superconformal transformations, $\delta_{\mathcal{H}}$, associated with a parameter $\Lambda^{\underline{a}}$. The covariant diffeomorphism $\delta_{\text {cgct }}(\xi)$ is related to the ordinary one $\delta_{\text {gct }}(\xi)$ by the rule [50]

$$
\delta_{\mathrm{cgct}}\left(\xi^{A}\right)=\delta_{\mathrm{gct}}\left(\xi^{A} E_{A}^{M}\right)-\delta_{\mathcal{H}}\left(\xi^{A} \omega_{A}^{\underline{a}}\right) .
$$

The closed three-form $\mathfrak{J}$ is required to transform by an exact three-form under the standard superconformal transformations,

$$
\delta_{\mathcal{H}} \mathfrak{J}=\mathrm{d} \Theta\left(\Lambda^{\underline{a}}\right) .
$$

If we assume the components $\xi^{M}$ and $\Lambda^{\underline{a}}$ vanish at spacetime infinity, then we have the supersymmetric invariant

$$
S=\int_{\mathcal{M}^{3}} i^{*} \mathfrak{J}
$$

Here $\mathcal{M}^{3}$ denotes the bosonic body of the curved superspace $\mathcal{M}^{3 \mid 2 \mathcal{N}}$ and $i: \mathcal{M}^{3} \rightarrow \mathcal{M}^{3 \mid 2 \mathcal{N}}$ is the inclusion map.

Suitable actions must also be gauge invariant for any additional gauge symmetries of the theory under consideration. If the closed three-form $\mathfrak{J}$ transforms by an exact threeform under the gauge transformations,

$$
\delta \mathfrak{J}=\mathrm{d} \Theta
$$

then the functional (3.5) is a suitable candidate for an action.

Our method to construct Chern-Simons actions is analogous to the one used in $[45,46]$ to derive the conformal supergravity actions for $\mathcal{N} \leq 6$. In the super Yang-Mills case, following [45], we will construct a closed three-form $\mathfrak{J}$ by finding two solutions to the superform equation

$$
\mathrm{d} \Sigma=\left\langle F^{2}\right\rangle:=\operatorname{tr}\{F \wedge F\} .
$$

The first of these solutions is the Chern-Simons three-form $\Sigma_{\mathrm{CS}}$,

$$
\Sigma_{\mathrm{CS}}=\operatorname{tr}\left\{V \wedge F-\frac{\mathrm{i}}{3} V \wedge V \wedge V\right\}
$$

It changes by an exact three-form under the Yang-Mills gauge transformation (2.2),

$$
\delta_{\tau} \Sigma_{\mathrm{CS}}=\mathrm{d} \operatorname{tr}\{\mathrm{id} \tau \wedge V\} .
$$

It is invariant under the standard superconformal transformations,

$$
\delta_{\mathcal{H}} \Sigma_{\mathrm{CS}}=0 .
$$

The other solution, the so-called curvature induced form $\Sigma_{R}$, is defined to be such that its components are constructed in terms of the field strength $F_{A B}$ and its covariant derivatives. 
This three-form is required to be invariant under the Yang-Mills gauge transformations (2.2) and under the the standard superconformal ones,

$$
\begin{aligned}
\delta_{\tau} \Sigma_{R} & =0, \\
\delta_{\mathcal{H}} \Sigma_{R} & =0 .
\end{aligned}
$$

The existence of $\Sigma_{R}$ is not guaranteed for arbitrary $\mathcal{N}$ and crucially depends on the explicit structure of the constraints obeyed by the field strength. If $\Sigma_{R}$ exists, the properties of $\Sigma_{\mathrm{CS}}$ and $\Sigma_{R}$ imply that their difference

$$
\mathfrak{J}=\Sigma_{\mathrm{CS}}-\Sigma_{R}=\operatorname{tr}\left\{V \wedge F-\frac{\mathrm{i}}{3} V \wedge V \wedge V\right\}-\Sigma_{R}
$$

is an appropriate closed three-form that constitutes a supersymmetric action.

We would like to emphasize that the three-form $\Sigma_{R}$ is required to be conformally invariant, eq. (3.11b). Actually, it turns out that the only non-trivial invariance condition on $\Sigma_{R}$ is with respect to the special conformal generators $K_{A}$. It is equivalent to the condition [45]

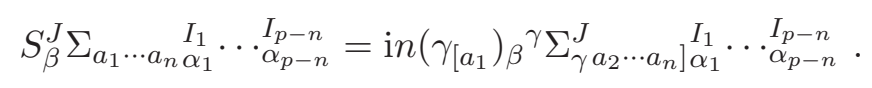

The above scheme is an example of a known construction where an invariant derived from a closed super $d$-form can be generated from a closed, gauge-invariant super $(d+1)$ form provided that the latter is Weil trivial, i.e. exact in invariant cohomology (a concept introduced by Bonora, Pasti and Tonin [71] in the context of anomalies in supersymmetric theories). Examples of this include higher-order invariants in other supersymmetric theories which were studied, e.g., in [72, 73].

\section{Non-Abelian curvature induced three-form}

We introduce the curvature induced form $\Sigma_{R}=\frac{1}{3 !} E^{C} \wedge E^{B} \wedge E^{A} \Sigma_{A B C}$ as the covariant solution to the superform equation ${ }^{7}$

$$
\mathrm{d} \Sigma_{R}=\operatorname{tr}\{F \wedge F\}, \quad 4 \nabla_{[A} \Sigma_{B C D\}}+6 T_{[A B}^{E} \Sigma_{|E| C D\}}=(\operatorname{tr}\{F \wedge F\})_{A B C D} .
$$

By covariant we mean that the components $\Sigma_{A B C}$ are directly expressible in terms of $F_{A B}$ and their covariant derivatives. It should be emphasized that the curvature induced form can only exist if the field strength $F$ is constrained in a such a way that eq. (4.1) can be satisfied.

To see this, consider the $\mathcal{N}>1$ case where one finds at the lowest dimension the condition

$$
E_{L}^{\delta} \wedge E_{K}^{\gamma} \wedge E_{J}^{\beta} \wedge E_{I}^{\alpha}\left(-24 \varepsilon_{\alpha \beta} \varepsilon_{\gamma \delta} \operatorname{tr}\left\{G^{I J} G^{K L}\right\}-4 \nabla_{\delta}^{L} \Sigma_{\alpha \beta \gamma}^{I J K}+12 \mathrm{i}\left(\gamma^{a}\right)_{\alpha \beta} \delta^{I J} \Sigma_{a \gamma \delta}^{K L}\right)=0 .
$$

\footnotetext{
${ }^{7}$ When referring to the components of the curvature induced form we will use $\Sigma$ instead of $\Sigma_{R}$ to avoid awkward notation.
} 
On dimensional grounds, the most general ansatz to take for $\Sigma_{R}$ is 8

$$
\Sigma_{\alpha \beta \gamma}^{I J K}=0, \quad \Sigma_{a \beta \gamma}^{J K}=\mathrm{i}\left(\gamma_{a}\right)_{\beta \gamma} \operatorname{tr}\left\{A \delta^{J K} G^{P Q} G_{P Q}+B G^{J L} G^{K}{ }_{L}\right\} .
$$

It will turn out that the curvature induced three-form, based on the ansatz (4.3), can only be found for $\mathcal{N}<4$. It is in these cases that we have

$$
\operatorname{tr}\left\{G^{I J} G^{K L}\right\}=A \delta^{K[I} \delta^{J] L} \operatorname{tr}\left\{G^{P Q} G_{P Q}\right\}+\frac{B}{2} \delta^{K[I} \operatorname{tr}\left\{G^{J] P} G^{L}{ }_{P}\right\}-\frac{B}{2} \delta^{L[I} \operatorname{tr}\left\{G^{J] P} G^{K}{ }_{P}\right\}
$$

for some $A$ and $B$.

Below we give the solution to eq. (4.1) on a case by case basis.

\subsection{The $\mathcal{N}=1$ case}

Since $F$ is constrained by eq. (2.5), solving (4.1) is straightforward. One finds ${ }^{9}$

$$
\begin{aligned}
\Sigma_{\alpha \beta \gamma} & =\Sigma_{a \beta \gamma}=\Sigma_{a b \gamma}=0, \\
\Sigma_{a b c} & =-\frac{\mathrm{i}}{4} \varepsilon_{a b c} \operatorname{tr}\left\{G^{\gamma} G_{\gamma}\right\} .
\end{aligned}
$$

Since the only non-zero component of this three-form is primary, $\Sigma_{R}$ is indeed conformally invariant by virtue of equation (3.11b).

\subsection{The $\mathcal{N}=2$ case}

In the $\mathcal{N}=2$ case, we can replace $G^{I J}$ with its Hodge-dual:

$$
G:=\frac{1}{2} \varepsilon^{I J} G_{I J}, \quad G^{I J}=\varepsilon^{I J} G .
$$

Then we have

$$
\operatorname{tr}\left\{G^{I J} G^{K L}\right\}=2 \delta^{I[K} \delta^{L] J} \operatorname{tr}\left\{G^{2}\right\}=\delta^{K[I} \delta^{J] L} \operatorname{tr}\left\{G^{P Q} G_{P Q}\right\} .
$$

Using the constraint (2.14) one finds the solution

$$
\begin{aligned}
\Sigma_{\alpha \beta \gamma}^{I J K} & =0, \\
\Sigma_{a \beta \gamma}^{J K} & =2 \mathrm{i}\left(\gamma_{a}\right)_{\beta \gamma} \delta^{J K} \operatorname{tr}\left\{G^{2}\right\}, \\
\Sigma_{a b \gamma} K & =-\varepsilon_{a b c}\left(\gamma^{c}\right)_{\gamma \delta} \operatorname{tr}\left\{\nabla^{\delta K} G^{2}\right\}, \\
\Sigma_{a b c} & =-\frac{\mathrm{i}}{2} \varepsilon_{a b c} \operatorname{tr}\left\{2 \nabla_{K}^{\gamma} G \nabla_{\gamma}^{K} G+G \nabla_{K}^{\gamma} \nabla_{\gamma}^{K} G\right\} .
\end{aligned}
$$

The curvature induced three-form can be shown to obey equation (3.11b).

It is often advantageous to make use of the complex basis for the $\mathcal{N}=2$ covariant derivatives, see $[50,52]$ for details. In this basis, the field strength is given by

$$
F=\bar{E}^{\beta} \wedge E^{\alpha} F_{\alpha \beta}+E^{\beta} \wedge E^{a} F_{a \beta}+\bar{E}^{\beta} \wedge E^{a} \bar{F}_{a \beta}+\frac{1}{2} E^{b} \wedge E^{a} F_{a b}
$$

\footnotetext{
${ }^{8}$ This is analogous to the ansatz taken for conformal supergravity [45].

${ }^{9}$ Keep in mind that eq. (4.1) is identically satisfied once it is solved up to and including the level of the highest dimension component, see [74].
} 
where its components are

$$
\begin{aligned}
F_{\alpha \beta} & =-2 \varepsilon_{\alpha \beta} G, \\
F_{a \beta} & =\mathrm{i}\left(\gamma_{a}\right)_{\beta}{ }^{\gamma} \nabla_{\gamma} G, \\
\bar{F}_{a \beta} & =-\mathrm{i}\left(\gamma_{a}\right)_{\beta}{ }^{\gamma} \bar{\nabla}_{\gamma} G, \\
F_{a b} & =-\frac{\mathrm{i}}{8} \varepsilon_{a b c}\left(\gamma^{c}\right)^{\gamma \delta}\left[\nabla_{\gamma}, \bar{\nabla}_{\delta}\right] G .
\end{aligned}
$$

The corresponding curvature induced form (4.8) may be expressed as

$$
\begin{aligned}
\Sigma= & \bar{E}^{\gamma} \wedge E^{\beta} \wedge E^{a} \Sigma_{a \beta \gamma}+\frac{1}{2} E^{\gamma} \wedge E^{b} \wedge E^{a} \Sigma_{a b \gamma}+\frac{1}{2} \bar{E}^{\gamma} \wedge E^{b} \wedge E^{a} \bar{\Sigma}_{a b \gamma} \\
& +\frac{1}{6} E^{c} \wedge E^{b} \wedge E^{a} \Sigma_{a b c},
\end{aligned}
$$

where the components have the explicit form

$$
\begin{aligned}
& \Sigma_{a \beta \gamma}=-2 \mathrm{i}\left(\gamma_{a}\right)_{\beta \gamma} \operatorname{tr}\left\{G^{2}\right\}, \\
& \Sigma_{a b \gamma}=-\varepsilon_{a b c}\left(\gamma^{c}\right)_{\gamma \delta} \operatorname{tr}\left\{\nabla^{\delta} G^{2}\right\}, \\
& \bar{\Sigma}_{a b \gamma}=-\varepsilon_{a b c}\left(\gamma^{c}\right)_{\gamma \delta} \operatorname{tr}\left\{\bar{\nabla}^{\delta} G^{2}\right\}, \\
& \Sigma_{a b c}=\mathrm{i} \varepsilon_{a b c} \operatorname{tr}\left\{2 \nabla^{\gamma} G \bar{\nabla}_{\gamma} G+G \bar{\nabla}^{\gamma} \nabla_{\gamma} G\right\} .
\end{aligned}
$$

\subsection{The $\mathcal{N}=3$ case}

In the $\mathcal{N}=3$ case we define the Hodge-dual of $G^{I J}$ as

$$
G_{I}:=\frac{1}{2} \varepsilon_{I J K} G^{J K}, \quad G^{I J}=\varepsilon^{I J K} G_{K},
$$

which implies

$$
\operatorname{tr}\left\{G^{I J} G^{K L}\right\}=2 \delta^{K[I} \delta^{J]} \operatorname{tr}\left\{G^{P} G_{P}\right\}-2 \delta^{K[I} \operatorname{tr}\left\{G^{J]} G^{L}\right\}+2 \delta^{L[I} \operatorname{tr}\left\{G^{J]} G^{K}\right\} .
$$

Furthermore, the Bianchi identity (2.15) gives

$$
\begin{aligned}
\nabla_{\gamma}^{I} G^{J} & =\nabla_{\gamma}^{[I} G^{J]}+\frac{1}{3} \delta^{I J} \nabla_{\gamma}^{K} G_{K}, \\
\nabla^{\gamma J} \nabla_{\gamma[J} G_{I]} & =2 \nabla^{\gamma J} \nabla_{\gamma J} G_{I}-8 \varepsilon_{I J K}\left[G^{J}, G^{K}\right], \\
\nabla_{\alpha}^{I} \nabla_{\beta}^{J} G_{J} & =-\frac{3}{2} \varepsilon_{\alpha \beta} \nabla_{P}^{\gamma} \nabla_{\gamma}^{P} G^{I}+3 \mathrm{i} \nabla_{\alpha \beta} G^{I}+9 \varepsilon_{\alpha \beta} \varepsilon^{I J K}\left[G_{J}, G_{K}\right] .
\end{aligned}
$$

Using the above identities one finds the solution

$$
\begin{aligned}
\Sigma_{\alpha \beta \gamma}^{I J K}= & 0, \\
\Sigma_{a \beta \gamma}^{J K}= & 2 \mathrm{i}\left(\gamma_{a}\right)_{\beta \gamma} \operatorname{tr}\left\{\delta^{J K} G^{I} G_{I}-2 G^{J} G^{K}\right\}, \\
\Sigma_{a b \gamma} K= & 2 \varepsilon_{a b c}\left(\gamma^{c}\right)_{\gamma}^{\delta} \operatorname{tr}\left\{\nabla_{\delta}^{[K} G^{I]} G_{I}-\frac{1}{3} \nabla_{\delta}^{I} G_{I} G^{K}\right\}, \\
\Sigma_{a b c}= & \frac{\mathrm{i}}{2} \varepsilon_{a b c} \operatorname{tr}\left\{\frac{2}{9}\left(\nabla^{\gamma K} G_{K}\right)\left(\nabla_{\gamma}^{L} G_{L}\right)-\left(\nabla_{[K}^{\gamma} G_{L]}\right)\left(\nabla_{\gamma}^{[K} G^{L]}\right)\right. \\
& \left.-2\left(\nabla_{K}^{\gamma} \nabla_{\gamma}^{K} G^{L}\right) G_{L}+8 \varepsilon^{I J K} G_{I} G_{J} G_{K}\right\} .
\end{aligned}
$$

Conformal invariance follows since $\Sigma_{R}$ obeys equation (3.11b). 


\subsection{The special case of $\mathcal{N}=4$}

In the previous subsections, we found that our approach struck an obstacle at the $\mathcal{N}=4$ case. In particular, eq. (4.4) no longer holds. Actually, the $\mathcal{N}=4$ case requires some additional consideration. It is well known that the constraint (2.15) does not define an irreducible off-shell supermultiplet for $\mathcal{N}=4$. In this case, the Hodge-dual of $G^{I J}$,

$$
\tilde{G}^{I J}:=\frac{1}{2} \varepsilon^{I J K L} G_{K L},
$$

obeys the same constraint as $G^{I J}$ does,

$$
\begin{aligned}
& \nabla_{\gamma}^{I} G^{J K}=\nabla_{\gamma}^{[I} G^{J K]}-\frac{2}{3} \delta^{I[J} \nabla_{\gamma L} G^{K] L}, \\
& \nabla_{\gamma}^{I} \tilde{G}^{J K}=\nabla_{\gamma}^{[I} \tilde{G}^{J K]}-\frac{2}{3} \delta^{I[J} \nabla_{\gamma L} \tilde{G}^{K] L} .
\end{aligned}
$$

As a result, one may constrain the field strength $G^{I J}$ to be self-dual,

$$
\tilde{G}^{I J}=G^{I J},
$$

or anti-self-dual,

$$
\tilde{G}^{I J}=-G^{I J}
$$

These choices correspond to two different off-shell $\mathcal{N}=4$ vector multiplets, the left and right ones, see [52] for more details.

Now, if we consider an irreducible $\mathcal{N}=4$ vector multiplet obeying either (4.19a) or (4.19b), it may be seen that eq. (4.4) still does not hold. A possible way out is to consider two vector multiplets and a generalization of eq. (3.7) of the form $\mathrm{d} \Sigma=\operatorname{tr}\left\{F_{1} \wedge F_{2}\right\}$. However, this poses a problem for non-Abelian vector multiplets, since the two-form field strengths $F_{1}$ and $F_{2}$ are not gauge invariant; instead, they transform covariantly under the two different gauge groups. ${ }^{10}$ In this section, we therefore restrict ourselves to Abelian vector multiplets.

We will consider the general case of two Abelian vector multiplets $G_{+}^{I J}$ and $G_{-}^{I J}$ with the two-form field strengths $F_{+}$and $F_{-}$respectively. In this case the superform equation (3.7) is replaced by

$$
\mathrm{d} \Sigma=F_{+} \wedge F_{-}, \quad 4 \nabla_{[A} \Sigma_{B C D\}}+6 T_{[A B}^{E} \Sigma_{|E| C D\}}=\left(F_{+} \wedge F_{-}\right)_{A B C D} .
$$

The Chern-Simons solution $\Sigma_{\mathrm{CS}}$ to the above is

$$
\Sigma_{\mathrm{CS}}=F_{+} \wedge V_{-}=V_{+} \wedge F_{-}+\text {closed form }
$$

where $V_{ \pm}$are gauge one-forms associated with the two-form field strengths, $F_{ \pm}=\mathrm{d} V_{ \pm}$.

\footnotetext{
${ }^{10}$ It should be mentioned that there is an alternative approach to the problem of constructing the $\mathcal{N}=4$ Chern-Simons action [75]. It is based on dualizing two scalars in the vector multiplet into vector fields and constructing a theory involving three different vectors! However, as mentioned in [58], such an approach is on-shell and cannot be used to construct matter couplings.
} 
The curvature induced three-form $\Sigma_{R}$ is the covariant solution to the superform equation (4.20) (when it exists). For $\mathcal{N}=4$ one finds at the lowest dimension of (4.20) the condition

$$
E_{L}^{\delta} \wedge E_{K}^{\gamma} \wedge E_{J}^{\beta} \wedge E_{I}^{\alpha}\left(-24 \varepsilon_{\alpha \beta} \varepsilon_{\gamma \delta} G_{+}^{I J} G_{-}^{K L}-4 \nabla_{\delta}^{L} \Sigma_{\alpha \beta \gamma}^{I J K}+12 \mathrm{i}\left(\gamma^{a}\right)_{\alpha \beta} \delta^{I J} \Sigma_{a \gamma \delta}^{K L}\right)=0 .
$$

The most general ansatz to take for $\Sigma_{R}$ is

$$
\Sigma_{\alpha \beta \gamma}^{I J K}=0, \quad \Sigma_{a \beta \gamma}^{J K}=\mathrm{i}\left(\gamma_{a}\right)_{\beta \gamma}\left(A \delta^{J K} G_{+}^{P Q} G_{-P Q}-B G_{+}^{L(J} G_{-}^{K)}\right),
$$

which will lead to a solution if

$$
G_{+}^{I J} G_{-}^{K L}=A \delta^{K[I} \delta^{J] L} G_{+}^{P Q} G_{-P Q}+\frac{B}{2} \delta^{K[I} G_{+}^{J] P} G_{-P}^{L}-\frac{B}{2} \delta^{L[I} G_{+}^{J] P} G_{-P}^{K} .
$$

It is easy to see that if we let both $G_{ \pm}^{I J}$ be (anti-)self-dual then we cannot satisfy eq. (4.24) for any $A$ and $B$. However, imposing opposite duality conditions gives us a way out. Taking $G_{+}^{I J}$ to be self-dual and $G_{-}^{I J}$ to be anti-self-dual,

$$
\frac{1}{2} \varepsilon^{I J K L} G_{ \pm K L}= \pm G_{ \pm}^{I J}
$$

gives $^{11}$

$$
G_{+}^{I J} G_{-}^{K L}=\delta^{K[I} G_{+}^{J] P} G_{-P}^{L}-\delta^{L[I} G_{+}^{J] P} G_{-P}^{K} .
$$

Using the Bianchi identity (2.15) and the (anti-)self-duality conditions (4.25), one finds the curvature induced form to be

$$
\begin{aligned}
\Sigma_{\alpha \beta \gamma}^{I J K} & =0 \\
\Sigma_{a \beta \gamma}^{J K} & =-2 \mathrm{i}\left(\gamma_{a}\right)_{\beta \gamma} G_{+}^{L(J} G_{-}^{K)} L \\
\Sigma_{a b \gamma} K & =-\frac{1}{3} \varepsilon_{a b c}\left(\gamma^{c}\right)_{\gamma}^{\delta}\left(\nabla_{\delta I} G_{+}^{I J} G_{-J}^{K}+\nabla_{\delta I} G_{-}^{I J} G_{+J}{ }^{K}\right), \\
\Sigma_{a b c} & =\mathrm{i} \varepsilon_{a b c}\left(\frac{1}{24} \nabla_{J}^{\gamma} \nabla_{\gamma}^{I} G_{+I K} G_{-}^{K J}+\frac{1}{24} \nabla_{J}^{\gamma} \nabla_{\gamma}^{I} G_{-I K} G_{+}^{K J}+\frac{1}{9} \nabla_{I}^{\gamma} G_{+}^{I J} \nabla_{\gamma}^{K} G_{-J K}\right) .
\end{aligned}
$$

One can check that eq. (3.13) holds.

As is known, the group isomorphism $\mathrm{SO}(4) \cong\left(\mathrm{SU}(2)_{\mathrm{L}} \times \mathrm{SU}(2)_{\mathrm{R}}\right) / \mathbb{Z}_{2}$ allows us to convert each $\mathrm{SO}(4)$ vector index into a pair of $\mathrm{SU}(2)$ spinor ones, for instance $\nabla_{\alpha}^{I} \rightarrow \nabla_{\alpha}^{i \bar{i}}$, see [52] for more details. It is instructive to look at some of the above results in the isospinor notation. The $\mathrm{SO}(4)$ bivector $G^{I J}=-G^{J I}$ is equivalently described by two symmetric second-rank isospinors, $G^{i j}$ and $G^{\overline{i j}}$, which are defined as

$$
G^{I J} \rightarrow G^{i \bar{i}, j \bar{j}}=-\varepsilon^{\overline{i j}} G^{i j}-\varepsilon^{i j} G^{\bar{i} \bar{j}}, \quad G^{i j}=G^{j i}, \quad G^{\bar{i} \bar{j}}=G^{\overline{j i}}
$$

and transform under the local groups $\mathrm{SU}(2)_{\mathrm{L}}$ and $\mathrm{SU}(2)_{\mathrm{R}}$, respectively. For the Hodge-dual $\mathrm{SO}(4)$ bivector $\tilde{G}^{I J}$ defined by (4.17), we get

$$
\tilde{G}^{I J} \rightarrow \tilde{G}^{i \bar{i}, j \bar{j}}=\varepsilon^{\overline{i j}} G^{i j}-\varepsilon^{i j} G^{\bar{i} \bar{j}} .
$$

\footnotetext{
${ }^{11}$ It is clear that $G_{+}^{I J} G_{-I J}=0$.
} 
The Bianchi identity (4.18a) is equivalent to the two analyticity constraints [52]

$$
\begin{aligned}
& \nabla_{\alpha}^{(i \bar{i}} G^{k l)}=0, \\
& \nabla_{\alpha}^{i(\bar{i}} G^{\bar{k} \bar{l})}=0 .
\end{aligned}
$$

Thus the field strengths $G^{i j}$ and $G^{\overline{i j}}$ are independent of each other. The (anti-)self-duality conditions (4.25) are equivalent to

$$
\begin{array}{ll}
G_{-}^{I J} \rightarrow G_{-}^{i \bar{i}, j \bar{j}}=-\varepsilon^{\bar{i} \bar{j}} G^{i j}, & G^{i j}=G^{j i}, \\
G_{+}^{I J} \rightarrow G_{+}^{i \bar{i}, j \bar{j}}=-\varepsilon^{i j} G^{\bar{i} \bar{j}}, & G^{\bar{i} \bar{j}}=G^{\overline{j i}} .
\end{array}
$$

In accordance with [52], a symmetric isospinor superfield $G^{i j}$ under the constraint (4.30a) is called a left linear multiplet or, equivalently, a left $\mathcal{O}(2)$ multiplet. Similarly, eq. (4.30b) defines a right linear multiplet or, equivalently, a right $\mathcal{O}(2)$ multiplet.

\section{Component actions}

In the previous sections we have given a complete superspace description of the ChernSimons actions for non-Abelian vector multiplets with $\mathcal{N}<4$ and of the $B F$ action for Abelian vector multiplets in the $\mathcal{N}=4$ case. In this section we will derive the corresponding component action. To do so we will need to elaborate on the component structure of the theory. For a complete description of the component fields of the Weyl multiplet including their supersymmetry transformations we refer the reader to [45]. Here we outline some of the salient details.

The Weyl multiplet contains a set of gauge one-forms which appear explicitly in the actions. These include the vielbein $e_{m}{ }^{a}$, the gravitino $\psi_{m_{I}}^{\alpha}$, the $\operatorname{SO}(\mathcal{N})$ gauge field $V_{m}{ }^{I J}$ and the dilatation gauge field $b_{m}$ defined as

$$
e_{m}^{a}:=E_{m}^{a}\left|, \quad \psi_{m I}^{\alpha}:=2 E_{m I}^{\alpha}\right|, \quad V_{m}^{I J}:=\Phi_{m}^{I J}\left|, \quad b_{m}:=B_{m}\right|,
$$

where the bar-projection $[54,76,77]$ of a superfield $V(z)=V(x, \theta)$ is defined by the standard rule $V|:=V(x, \theta)|_{\theta=0}$. The remaining gauge fields are the spin connection $\omega_{m}{ }^{a b}$, the special conformal and $S$-supersymmetry connections $\mathfrak{f}_{m}{ }^{a}$ and $\phi_{m_{\alpha}}^{I}$ defined as

$$
\omega_{m}^{a b}:=\Omega_{m}^{a b}\left|, \quad \mathfrak{f}_{m}{ }^{a}:=\mathfrak{F}_{m}{ }^{a}\right|, \quad \phi_{m_{\alpha}}^{I}:=2 \mathfrak{F}_{m_{\alpha}}^{I} \mid .
$$

These connections turn out to be composite and their expressions are given in [45].

The Weyl multiplet also contains some auxiliary fields for $\mathcal{N}>2$. In the $\mathcal{N}=3$ case, there is a single fermionic auxiliary field defined by

$$
w_{\alpha}=W_{\alpha} \mid \text {. }
$$

In the $\mathcal{N}=4$ case, the Weyl multiplet contains both bosonic and fermionic auxiliary fields,

$$
w=W\left|, \quad y=-\frac{\mathrm{i}}{4} \nabla_{I}^{\alpha} \nabla_{\alpha}^{I} W\right|, \quad w_{\alpha}^{I}=-\frac{\mathrm{i}}{2} \nabla_{\alpha}^{I} W \mid,
$$

where $W$ denotes the Hodge-dual of $W^{I J K L}$,

$$
W^{I J K L}=\varepsilon^{I J K L} W .
$$




\subsection{Vector multiplets in components}

The component fields of vector multiplets may be extracted from the field strength $G^{I J}$. For $\mathcal{N}>1$, we define the matter fields as follows ${ }^{12}$

$$
\begin{aligned}
g^{I J} & :=G^{I J} \mid, \\
\lambda_{\alpha}^{I} & :=\frac{2}{\mathcal{N}-1} \nabla_{\alpha J} G^{I J} \mid, \\
h^{I J} & :=\frac{\mathrm{i}}{\mathcal{N}-1} \nabla^{\gamma[I} \nabla_{\gamma K} G^{J] K} \mid, \\
\chi_{\alpha_{1} \cdots \alpha_{n}}^{I_{1} \cdots I_{n+2}} & :=I(n) \nabla_{\left(\alpha_{1}\right.}^{\left[I_{1}\right.} \cdots \nabla_{\left.\alpha_{n}\right)}^{I_{n}} G^{\left.I_{n+1} I_{n+2}\right]} \mid,
\end{aligned}
$$

where

$$
I(n)= \begin{cases}\mathrm{i}, & n=1,2(\bmod 4) \\ 1, & n=3,4(\bmod 4)\end{cases}
$$

A final component field $v_{m}$ is given by the bar-projection of the corresponding superspace connection,

$$
v_{a}=e_{a}^{m} v_{m}, \quad v_{m}:=V_{m} \mid .
$$

The covariant field strength may be constructed from the bar-projection of the twoform $F=\frac{1}{2} E^{B} \wedge E^{A} F_{A B}$. Making use of the identity

$$
F_{m n}=E_{m}{ }^{A} E_{n}{ }^{B} F_{A B}(-1)^{\varepsilon_{A} \varepsilon_{B}}
$$

and performing a component projection, we find

$$
\hat{F}_{a b}:=F_{a b} \mid=f_{a b}+\frac{1}{2}\left(\psi_{[a}^{K} \gamma_{b]} \lambda_{K}\right)-\frac{\mathrm{i}}{2} \psi_{a}{ }^{\gamma K} \psi_{b \gamma}^{L} g_{K L},
$$

where

$$
f_{a b}=e_{a}^{m} e_{b}^{n} f_{m n}, \quad f_{m n}:=F_{m n}\left|=2\left(\partial_{[m} V_{n]}-\mathrm{i} V_{[m} V_{n]}\right)\right|=2\left(\partial_{[m} v_{n]}-\mathrm{i} v_{[m} v_{n]}\right) .
$$

The component fields of the vector multiplet form a tower [70], see figure $1 .{ }^{13}$

The coefficients chosen in eq. (5.6) allow for a straightforward truncation of higher $\mathcal{N}$ cases to the lower $\mathcal{N}$ ones via a procedure analogous to the one described in [45]. For the $\mathcal{N}=1$ case we have to switch off all matter fields except

$$
\lambda_{\alpha}^{I}=\lambda_{\alpha}=G_{\alpha} \mid
$$

with the field strength $G_{\alpha}$ defined in (2.6a).

The $\mathcal{N}=4$ case is special, since it allows for two inequivalent off-shell vector multiplets with field strengths $G_{+}^{I J}$ and $G_{-}^{I J}$ obeying the self-duality condition (4.19a) and the antiself-duality condition (4.19b) respectively. In this case we define the the component fields

\footnotetext{
${ }^{12}$ The coefficients are chosen so that the $\mathcal{N}=1$ case may be derived via the higher $\mathcal{N}$ cases.

${ }^{13}$ The tower is analogous to the one for the $\mathcal{N}$-extended super Cotton tensor [45, 70].
} 


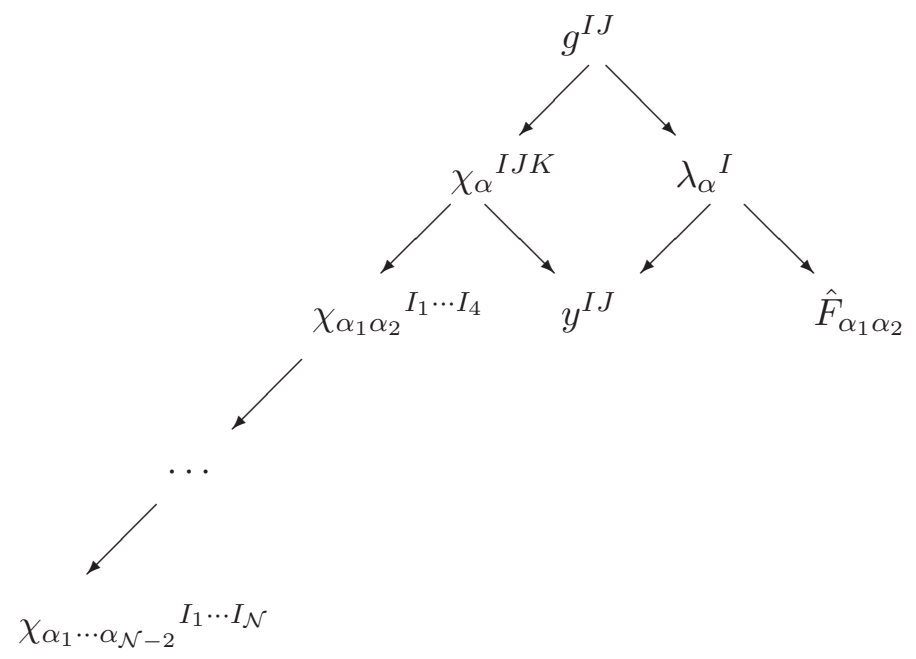

Figure 1. Component fields of the $\mathcal{N}$-extended vector multiplet.

of the vector multiplets as

$$
\begin{aligned}
g_{ \pm}^{I J} & :=G_{ \pm}^{I J} \mid, \\
\lambda_{( \pm) \alpha}^{I} & :=\frac{2}{3} \nabla_{\alpha J} G_{ \pm}^{I J} \mid, \\
h_{( \pm)}^{I J} & :=\frac{\mathrm{i}}{3} \nabla^{\gamma\left[{ }^{I}\right.} \nabla_{\gamma K} G_{ \pm}^{J] K} \mid, \\
\chi_{( \pm) \alpha_{1} \cdots \alpha_{n}}^{I_{1} \cdots I_{n+2}} & :=I(n) \nabla_{\left(\alpha_{1}\right.}^{\left[I_{1}\right.} \cdots \nabla_{\left.\alpha_{n}\right)}^{I_{n}} G_{ \pm}^{\left.I_{n+1} I_{n+2}\right]} \mid, \quad n=1,2,
\end{aligned}
$$

where $g_{ \pm}{ }^{I J}$ is (anti-)self-dual

$$
\frac{1}{2} \varepsilon^{I J K L} g_{ \pm K L}= \pm g_{ \pm}{ }^{I J}
$$

The component one-forms are given by

$$
v_{( \pm) a}=e_{a}^{m} v_{( \pm) m}, \quad v_{( \pm) m}:=V_{ \pm m} \mid,
$$

where $V_{ \pm}$is the gauge one-form associated with the field strength $G_{ \pm}^{I J}$.

The (anti-)self-duality property of $G_{ \pm}^{I J}$, eq. (4.25), reduces the degrees of freedom for each vector multiplet by half. To see this, it is useful to replace $h_{( \pm)}^{I J}$ by the fields

$$
\begin{aligned}
\hat{h}_{ \pm}{ }^{I J} & =\frac{1}{2}\left(h_{(\mp)}{ }^{I J}+\tilde{h}_{(\mp)}{ }^{I J}\right) \\
& =h_{(\mp)}{ }^{I J} \mp 2 w g_{\mp}{ }^{I J} \mp \frac{\mathrm{i}}{4}\left[g_{+}{ }^{P[I}, g_{-}{ }^{J]} P\right],
\end{aligned}
$$

which prove to be (anti-)self-dual

$$
\frac{1}{2} \varepsilon^{I J K L} \hat{h}_{ \pm K L}= \pm \hat{h}_{ \pm}^{I J}
$$


The other implications of (anti-)self-duality are

$$
\begin{aligned}
\chi_{ \pm \alpha}^{I J K} & =\mp \frac{\mathrm{i}}{2} \varepsilon^{I J K L} \lambda_{ \pm \alpha L}, \\
\chi_{ \pm \alpha \beta}^{I J K L} & =\mp \varepsilon^{I J K L} \hat{F}_{ \pm \alpha \beta} .
\end{aligned}
$$

Diagrammatically, this means that the components on the left hand side of figure 1 are related to those on the right hand side via (anti-)self-duality. One can see that each vector multiplet constitutes $8+8$ degrees of freedom.

\subsection{Off-shell component actions}

Now we have all the ingredients to construct the component actions corresponding to the closed forms

$$
\mathfrak{J}=\Sigma_{\mathrm{CS}}-\Sigma_{R}
$$

found in the previous sections. To do so we just need to apply the action principle (3.5),

$$
S=\left.\int \mathrm{d}^{3} x e^{*} \mathfrak{J}\right|_{\theta=0}, \quad{ }^{*} \mathfrak{J}=\frac{1}{3 !} \varepsilon^{m n p} \mathfrak{J}_{m n p}, \quad e=\operatorname{det}\left(e_{m}{ }^{a}\right),
$$

and make use of the formula

$$
\begin{aligned}
\frac{1}{3 !} \varepsilon^{m n p} \Sigma_{m n p} \mid= & \frac{1}{3 !} \varepsilon^{m n p} E_{p}{ }^{C} E_{n}{ }^{B} E_{m}{ }^{A} \Sigma_{A B C} \mid \\
= & \frac{1}{3 !} \varepsilon^{a b c}\left(\Sigma_{a b c}\left|+\frac{3}{2} \psi_{a I}^{\alpha} \Sigma_{\alpha b c}^{I}\right|+\frac{3}{4} \psi_{b J}^{\beta} \psi_{a I}^{\alpha} \Sigma_{\alpha \beta c}^{I J} \mid\right. \\
& \left.+\frac{1}{8} \psi_{c K}^{\gamma} \psi_{b}^{\beta} \psi_{a I}^{\alpha} \Sigma_{\alpha \beta \gamma}^{I J K} \mid\right) .
\end{aligned}
$$

Here we present the resulting actions on a case by case basis.

Although all our actions are automatically supersymmetric, we give the supersymmetry transformations of the component fields in appendix B.

\subsubsection{The non-Abelian $\mathcal{N}=1$ case}

The action is constructed using eqs. (3.8) and (4.5). From eq. (5.20) we find

$$
\frac{1}{3 !} \varepsilon^{m n p} \Sigma_{m n p} \mid=\frac{\mathrm{i}}{4} \operatorname{tr}\left\{\lambda^{\gamma} \lambda_{\gamma}\right\}
$$

Combining this with the contribution coming from the Chern-Simons form (3.8) gives

$$
S=\frac{1}{2} \int \mathrm{d}^{3} x e \operatorname{tr}\left\{\varepsilon^{a b c}\left(v_{a} f_{b c}+\frac{2 \mathrm{i}}{3} v_{a} v_{b} v_{c}\right)-\frac{\mathrm{i}}{2} \lambda^{\gamma} \lambda_{\gamma}\right\} .
$$

\subsubsection{The non-Abelian $\mathcal{N}=2$ case}

Using eqs. (5.20) and (4.8) we find

$$
\frac{1}{3 !} \varepsilon^{m n p} \Sigma_{m n p} \mid=\frac{1}{2} \operatorname{tr}\left\{\frac{\mathrm{i}}{2} \tilde{\lambda}_{K}^{\gamma} \tilde{\lambda}_{\gamma}^{K}-2 g h\right\}+\frac{1}{2}\left(\gamma^{a}\right)_{\gamma \delta} \psi_{a}{ }_{I}^{\gamma} \operatorname{tr}\left\{g \tilde{\lambda}^{\delta I}\right\}-\frac{\mathrm{i}}{4} \varepsilon^{a b c}\left(\gamma_{a}\right)_{\gamma \delta} \psi_{b}^{\gamma}{ }_{K}^{\gamma}{ }_{c}^{\delta K} \operatorname{tr}\left\{g^{2}\right\},
$$


where we have defined

$$
\tilde{\lambda}_{\alpha I}:=\varepsilon_{J I} \lambda_{\alpha}^{J}=-2 \nabla_{\alpha I} G\left|, \quad h:=\frac{1}{2} \varepsilon_{I J} h^{I J}=-\frac{\mathrm{i}}{2} \nabla^{\gamma K} \nabla_{\gamma K} G\right| .
$$

Using the above result and incorporating the Chern-Simons form (3.8) gives the action

$$
\begin{aligned}
S= & \frac{1}{2} \int \mathrm{d}^{3} x e \operatorname{tr}\left\{\varepsilon^{a b c}\left(v_{a} f_{b c}+\frac{2 \mathrm{i}}{3} v_{a} v_{b} v_{c}\right)-\frac{\mathrm{i}}{2} \tilde{\lambda}_{K}^{\gamma} \tilde{\lambda}_{\gamma}^{K}+2 g h-\left(\gamma^{a}\right)_{\gamma \delta} \psi_{a}^{\gamma} g \tilde{\lambda}^{\delta I}\right. \\
& \left.+\frac{\mathrm{i}}{2} \varepsilon^{a b c}\left(\gamma_{a}\right)_{\gamma \delta} \psi_{b}^{\gamma}{ }_{K}^{\gamma} \psi_{c}^{\delta K} g^{2}\right\} .
\end{aligned}
$$

Expressing the above action in terms of the complex basis gives

$$
\begin{aligned}
S= & \frac{1}{2} \int \mathrm{d}^{3} x e \operatorname{tr}\left\{\varepsilon^{a b c} v_{a} f_{b c}+\mathrm{i} \lambda^{\gamma} \bar{\lambda}_{\gamma}+2 g h\right. \\
& \left.-\left(\gamma^{a}\right)_{\gamma \delta} \psi_{a}^{\gamma} g \lambda^{\delta}+\left(\gamma^{a}\right)_{\gamma \delta} \bar{\psi}_{a}^{\gamma} g \bar{\lambda}^{\delta}-\mathrm{i} \varepsilon^{a b c}\left(\gamma_{a}\right)_{\gamma \delta} \psi_{b}^{\gamma} \bar{\psi}_{c}^{\delta} g^{2}\right\},
\end{aligned}
$$

where we have made use of the component fields in the complex basis:

$$
\lambda_{\alpha}=-2 \nabla_{\alpha} G\left|, \quad \bar{\lambda}_{\alpha}=-2 \bar{\nabla}_{\alpha} G\right|, \quad h=\mathrm{i} \bar{\nabla}^{\gamma} \nabla_{\gamma} G \mid .
$$

\subsubsection{The non-Abelian $\mathcal{N}=3$ case}

Using eqs. (5.20) and (4.16) we find

$$
\begin{aligned}
\frac{1}{3 !} \varepsilon^{m n p} \Sigma_{m n p} \mid= & -\frac{\mathrm{i}}{2} \operatorname{tr}\left\{-2 \chi^{\gamma} \chi_{\gamma}-\frac{1}{4} \lambda^{\gamma I J} \lambda_{\gamma I J}-2 \mathrm{i} h^{I} g_{I}-8 \varepsilon^{I J K} g_{I} g_{J} g_{K}\right\} \\
& +\frac{1}{2}\left(\gamma^{a}\right)_{\gamma \delta} \psi_{a}^{\gamma} \operatorname{tr}\left\{\lambda^{\delta I J} g_{J}+2 \mathrm{i} \chi^{\delta} g^{I}\right\} \\
& -\frac{\mathrm{i}}{4} \varepsilon^{a b c}\left(\gamma_{a}\right)_{\gamma \delta} \psi_{b}^{\gamma} \psi_{c L}^{\delta} \operatorname{tr}\left\{\delta^{K L} g^{P} g_{P}-2 g^{K} g^{L}\right\}
\end{aligned}
$$

where we have defined

$$
\begin{aligned}
g_{I} & :=\frac{1}{2} \varepsilon_{I J K} G^{J K}\left|=G_{I}\right|, \\
\lambda_{\alpha}{ }^{I J} & :=\varepsilon^{I J K} \lambda_{\alpha K}=2 \nabla_{\alpha}^{[I} G^{J]} \mid \\
\chi_{\alpha} & :=\frac{1}{3 !} \varepsilon_{I J K} \chi_{\alpha}^{I J K}=\frac{\mathrm{i}}{3} \nabla_{\alpha}^{I} G_{I} \mid, \\
h_{I} & :=\frac{1}{2 !} \varepsilon_{I J K} h^{J K}=\frac{\mathrm{i}}{2} \nabla^{\gamma J} \nabla_{\gamma[I} G_{J]}\left|=-\mathrm{i} \nabla^{\gamma J} \nabla_{\gamma J} G_{I}\right|+8 \mathrm{i} \varepsilon_{I J K} g^{J} g^{K} .
\end{aligned}
$$

Combining this with the contribution coming from the Chern-Simons form (3.8) gives

$$
\begin{aligned}
S= & \frac{1}{2} \int \mathrm{d}^{3} x e \operatorname{tr}\left\{\varepsilon^{a b c}\left(v_{a} f_{b c}+\frac{2 \mathrm{i}}{3} v_{a} v_{b} v_{c}\right)-2 \mathrm{i} \chi^{\gamma} \chi_{\gamma}-\frac{\mathrm{i}}{4} \lambda^{\gamma I J} \lambda_{\gamma I J}+2 g^{I} h_{I}-8 \mathrm{i} \varepsilon^{I J K} g_{I} g_{J} g_{K}\right. \\
& -\left(\gamma^{a}\right)_{\gamma \delta} \psi_{a}^{\gamma}\left(\lambda^{\delta I J} g_{J}+2 \mathrm{i} \chi^{\delta} g^{I}\right) \\
& \left.+\frac{\mathrm{i}}{2} \varepsilon^{a b c}\left(\gamma_{a}\right)_{\gamma \delta} \psi_{b}{ }_{K}^{\gamma} \psi_{c L}^{\delta}\left(\delta^{K L} g^{P} g_{P}-2 g^{K} g^{L}\right)\right\} .
\end{aligned}
$$


As in [45], our choice of normalization for the component fields allows for a simple truncation to the actions for lower values of $\mathcal{N}$. For example, from the above action one can truncate the auxiliary fields to $\mathcal{N}=2$ by taking (with $I, J=1,2$ )

$$
\left.\begin{array}{c}
g_{I} \longrightarrow 0, \quad \lambda_{\alpha}{ }^{I J} \longrightarrow 0, \quad \chi_{\alpha} \longrightarrow 0, \quad h^{I} \longrightarrow 0, \\
g^{3} \longrightarrow g, \quad \lambda_{\alpha}{ }^{I 3} \longrightarrow \tilde{\lambda}_{\alpha}{ }^{I}, \quad h^{3} \longrightarrow h
\end{array}\right\}
$$

For the fields of the Weyl multiplet one performs a similar truncation, which is given in [45].

\subsubsection{The Abelian $\mathcal{N}=4$ case}

Using eqs. (5.20) and (4.27) we find

$$
\begin{aligned}
\frac{1}{3 !} \varepsilon^{m n p} \Sigma_{m n p}= & -\frac{1}{8} \hat{h}_{+}^{I J} g_{-I J}-\frac{1}{8} \hat{h}_{-}^{I J} g_{+I J}+\frac{\mathrm{i}}{4} \lambda_{(+)}^{\alpha I} \lambda_{(-) \alpha I} \\
& +\frac{1}{4}\left(\gamma^{a}\right)_{\gamma \delta} \psi_{a}{ }_{I}^{\gamma}\left(\lambda_{(+)}^{\delta J} g_{-J}{ }^{I}+\lambda_{(-)}^{\delta J} g_{+J}{ }^{I}\right) \\
& -\frac{\mathrm{i}}{4} \varepsilon^{a b c}\left(\gamma_{a}\right)_{\gamma \delta} \psi_{b}{ }_{K} \psi_{c L}^{\delta} g_{+}{ }^{K P}{ }_{g_{-}}{ }^{L} P .
\end{aligned}
$$

Combining this with the contribution coming from the Chern-Simons form (4.21) gives

$$
\begin{aligned}
S= & \frac{1}{2} \int \mathrm{d}^{3} x e\left(\varepsilon^{a b c} v_{(+) a} f_{(-) b c}+\frac{1}{4} \hat{h}_{+}{ }^{I J} g_{+I J}+\frac{1}{4} \hat{h}_{-}{ }^{I J} g_{-I J}-\frac{\mathrm{i}}{2} \lambda_{(+)}^{\alpha I} \lambda_{(-) \alpha I}\right. \\
& -\frac{1}{2}\left(\gamma^{a}\right)_{\gamma \delta} \psi_{a I}^{\gamma}\left(\lambda_{(+)}^{\delta J} g_{-J}{ }^{I}+\lambda_{(-)}^{\delta J} g_{+J}{ }^{I}\right) \\
& \left.+\frac{\mathrm{i}}{2} \varepsilon^{a b c}\left(\gamma_{a}\right)_{\gamma \delta} \psi_{b_{K}}^{\gamma} \psi_{c L}^{\delta} g_{+}{ }^{K P} g_{-}{ }^{L}{ }_{P}\right) .
\end{aligned}
$$

\section{Matter-coupled $\mathcal{N}=2$ supergravity}

The results of sections 4 and 5 may be used to generate locally supersymmetric actions. This idea can be illustrated, in a simple and transparent way, by considering the $\mathcal{N}=2$ case which we discuss below. Unlike in section 5 , here we use the complex basis for the $\mathcal{N}=2$ covariant derivatives, see [52] for details.

Let us consider a locally supersymmetric $B F$ term described by the action

$$
S_{B F}=\int \mathrm{d}^{3} x \mathrm{~d}^{2} \theta \mathrm{d}^{2} \bar{\theta} E \mathcal{V} \boldsymbol{G}, \quad E^{-1}=\operatorname{Ber}\left(E_{A}^{M}\right) .
$$

Here $\mathcal{V}=\overline{\mathcal{V}}$ is the gauge prepotential of an Abelian vector multiplet, and $\boldsymbol{G}=\overline{\boldsymbol{G}}$ a real linear superfield, $\nabla^{2} \boldsymbol{G}=\bar{\nabla}^{2} \boldsymbol{G}=0 .{ }^{14}$ The action (6.1) is invariant under gauge transformations

$$
\delta \mathcal{V}=\lambda+\bar{\lambda}, \quad \bar{\nabla}_{\alpha} \lambda=0
$$

with the gauge parameter $\lambda$ being an arbitrary covariantly chiral dimensionless scalar. Eq. (6.1) defines the $\mathcal{N}=2$ linear multiplet action.

\footnotetext{
${ }^{14}$ The constraints on $\boldsymbol{G}$ may be solved as $\boldsymbol{G}=\mathrm{i} \nabla^{\alpha} \bar{\nabla}_{\alpha} \mathcal{V}$, for some $\mathcal{V}$. In certain cases, $\mathcal{V}$ is not a well-defined local operator.
} 
It turns out that the action (6.1) may be recast in terms of a closed three-form

$$
J=V \wedge \boldsymbol{F}-\Sigma, \quad \mathrm{d} J=0
$$

that involves three building blocks. First of all, $\boldsymbol{F}=\frac{1}{2} E^{B} \wedge E^{A} \boldsymbol{F}_{A B}$ is a closed two-form, $\mathrm{d} \boldsymbol{F}=0$, associated with $\boldsymbol{G}$. Its components are defined as in eqs. (4.9) and (4.10) by

$$
\boldsymbol{F}=\bar{E}^{\beta} \wedge E^{\alpha} \boldsymbol{F}_{\alpha \beta}+E^{\beta} \wedge E^{a} \boldsymbol{F}_{a \beta}+\bar{E}^{\beta} \wedge E^{a} \overline{\boldsymbol{F}}_{a \beta}+\frac{1}{2} E^{b} \wedge E^{a} \boldsymbol{F}_{a b},
$$

and are explicitly given as follows:

$$
\begin{aligned}
\boldsymbol{F}_{\alpha \beta} & =-2 \varepsilon_{\alpha \beta} \boldsymbol{G}, \\
\boldsymbol{F}_{a \beta} & =\mathrm{i}\left(\gamma_{a}\right)_{\beta} \nabla_{\gamma} \boldsymbol{G}, \\
\overline{\boldsymbol{F}}_{a \beta} & =-\mathrm{i}\left(\gamma_{a}\right)_{\beta}{ }^{\gamma} \bar{\nabla}_{\gamma} \boldsymbol{G}, \\
\boldsymbol{F}_{a b} & =-\frac{\mathrm{i}}{8} \varepsilon_{a b c}\left(\gamma^{c}\right)^{\gamma \delta}\left[\nabla_{\gamma}, \bar{\nabla}_{\delta}\right] \boldsymbol{G} .
\end{aligned}
$$

The second building block, $V=E^{A} V_{A}$, is the gauge one-form describing the vector multiplet associated with $\mathcal{V}$. Modulo an exact one-form, we can choose the components of $V$ as follows:

$$
V_{\alpha}=\mathrm{i} \nabla_{\alpha} \mathcal{V}, \quad \bar{V}_{\alpha}=-\mathrm{i} \bar{\nabla}_{\alpha} \mathcal{V}, \quad V_{a}=-\frac{1}{4}\left(\gamma_{a}\right)^{\alpha \beta}\left[\nabla_{\alpha}, \bar{\nabla}_{\beta}\right] \mathcal{V}
$$

The corresponding gauge-invariant field strength $F=\mathrm{d} V$ has the explicit structure given by eqs. (6.4) and (6.5) with $\boldsymbol{F}$ and $\boldsymbol{G}$ replaced with $F$ and $G$ respectively, where $G$ denotes the gauge-invariant field strength

$$
G=\mathrm{i} \nabla^{\alpha} \bar{\nabla}_{\alpha} \mathcal{V}
$$

associated with the prepotential $\mathcal{V}$. Finally, the three-form $\Sigma$ is chosen to obey the equation

$$
\mathrm{d} \Sigma=F \wedge \boldsymbol{F} .
$$

Its components are defined by

$$
\begin{aligned}
\Sigma= & \bar{E}^{\gamma} \wedge E^{\beta} \wedge E^{a} \Sigma_{a \beta \gamma}+\frac{1}{2} E^{\gamma} \wedge E^{b} \wedge E^{a} \Sigma_{a b \gamma}+\frac{1}{2} \bar{E}^{\gamma} \wedge E^{b} \wedge E^{a} \bar{\Sigma}_{a b \gamma} \\
& +\frac{1}{6} E^{c} \wedge E^{b} \wedge E^{a} \Sigma_{a b c}
\end{aligned}
$$

and have the following explicit form:

$$
\begin{aligned}
\Sigma_{a \beta \gamma} & =-2 \mathrm{i}\left(\gamma_{a}\right)_{\beta \gamma} G \boldsymbol{G}, \\
\Sigma_{a b \gamma} & =-\varepsilon_{a b c}\left(\gamma^{c}\right)_{\gamma \delta}\left(\boldsymbol{G} \nabla^{\delta} G+G \nabla^{\delta} \boldsymbol{G}\right), \\
\bar{\Sigma}_{a b \gamma} & =-\varepsilon_{a b c}\left(\gamma^{c}\right)_{\gamma \delta}\left(\boldsymbol{G} \bar{\nabla}^{\delta} G+G \bar{\nabla}^{\delta} \boldsymbol{G}\right), \\
\Sigma_{a b c} & =\frac{\mathrm{i}}{2} \varepsilon_{a b c}\left(4 \nabla^{\gamma} \boldsymbol{G} \bar{\nabla}_{\gamma} G+\boldsymbol{G} \bar{\nabla}^{\gamma} \nabla_{\gamma} G+G \bar{\nabla}^{\gamma} \nabla_{\gamma} \boldsymbol{G}\right) .
\end{aligned}
$$

The components of $\Sigma$ are symmetric under the interchange $G \leftrightarrow \boldsymbol{G}$. When $G=\boldsymbol{G}$ we have agreement with eqs. (4.11) and (4.12) in the Abelian case. 
Associated with the closed three-form $J$, eq. (6.3), is the component action

$$
\begin{aligned}
S= & \frac{1}{2} \int \mathrm{d}^{3} x e\left(\varepsilon^{a b c} v_{a} \boldsymbol{f}_{b c}+\frac{\mathrm{i}}{2} \lambda^{\gamma} \overline{\boldsymbol{\lambda}}_{\gamma}+\frac{\mathrm{i}}{2} \boldsymbol{\lambda}^{\gamma} \bar{\lambda}_{\gamma}+g \boldsymbol{h}+\boldsymbol{g} h\right. \\
& -\frac{1}{2}\left(\gamma^{a}\right)_{\gamma \delta} \psi_{a}^{\gamma}\left(g \boldsymbol{\lambda}^{\delta}+\boldsymbol{g} \lambda^{\delta}\right)+\frac{1}{2}\left(\gamma^{a}\right)_{\gamma \delta} \bar{\psi}_{a}^{\gamma}\left(g \overline{\boldsymbol{\lambda}}^{\delta}+\boldsymbol{g} \bar{\lambda}^{\delta}\right) \\
& \left.-\mathrm{i} \varepsilon^{a b c}\left(\gamma_{a}\right)_{\gamma \delta} \psi_{b}{ }^{\gamma} \bar{\psi}_{c}^{\delta} g \boldsymbol{g}\right),
\end{aligned}
$$

where the component fields are defined as in section 5 (in the complex basis):

$$
\begin{aligned}
g & =G\left|, \quad \lambda_{\alpha}=-2 \nabla_{\alpha} G\right|, \quad \bar{\lambda}_{\alpha}=-2 \bar{\nabla}_{\alpha} G\left|, \quad h=\mathrm{i} \bar{\nabla}^{\gamma} \nabla_{\gamma} G\right|, \\
\boldsymbol{g} & =\boldsymbol{G}\left|, \quad \boldsymbol{\lambda}_{\alpha}=-2 \nabla_{\alpha} \boldsymbol{G}\right|, \quad \overline{\boldsymbol{\lambda}}_{\alpha}=-2 \bar{\nabla}_{\alpha} \boldsymbol{G}\left|, \quad \boldsymbol{h}=\mathrm{i} \bar{\nabla}^{\gamma} \nabla_{\gamma} \boldsymbol{G}\right|, \\
v_{a} & =e_{a}{ }^{m} V_{m} \mid, \\
\boldsymbol{f}_{a b} & =\boldsymbol{F}_{a b}\left|-\psi_{[a}{ }^{\beta} \boldsymbol{F}_{b] \beta}\right|-\bar{\psi}_{[a}{ }^{\beta} \overline{\boldsymbol{F}}_{b] \beta} \mid-\frac{1}{2} \psi_{[a}{ }^{\alpha} \bar{\psi}_{b]}{ }^{\beta} \boldsymbol{F}_{\alpha \beta} \\
& =-\frac{\mathrm{i}}{8} \varepsilon_{a b c}\left(\gamma^{c}\right)^{\gamma \delta}\left[\nabla_{\gamma}, \bar{\nabla}_{\delta}\right] \boldsymbol{G} \mid+\frac{\mathrm{i}}{2} \psi_{[a}{ }^{\beta}\left(\gamma_{b]}\right)_{\beta}{ }^{\gamma} \boldsymbol{\lambda}_{\gamma}-\frac{\mathrm{i}}{2} \bar{\psi}_{[a}{ }^{\beta}\left(\gamma_{b]}\right)_{\beta}{ }^{\gamma} \overline{\boldsymbol{\lambda}}_{\gamma}+\psi_{[a}{ }^{\alpha} \bar{\psi}_{b] \alpha} \boldsymbol{g} .
\end{aligned}
$$

Eq. (6.11) is exactly the component form of the action (6.1).

Let us recall that the most general $\mathcal{N}=2$ supergravity-matter system (see $[52,78]$ for more details) is described by an action of the form

$$
S=\int \mathrm{d}^{3} x \mathrm{~d}^{2} \theta \mathrm{d}^{2} \bar{\theta} E \mathcal{L}+\int \mathrm{d}^{3} x \mathrm{~d}^{2} \theta \mathcal{E} \mathcal{L}_{\mathrm{c}}+\int \mathrm{d}^{3} x \mathrm{~d}^{2} \bar{\theta} \overline{\mathcal{E}} \overline{\mathcal{L}}_{\mathrm{c}}
$$

for some real scalar $\mathcal{L}$ and covariantly chiral scalar $\mathcal{L}_{\mathrm{c}}$ Lagrangians, $\bar{\nabla}_{\alpha} \mathcal{L}_{\mathrm{c}}=0$. Here $\mathcal{E}$ denotes the chiral density. ${ }^{15}$ We assume that the dynamical supermultiplets include an Abelian vector multiplet described by prepotential $\mathcal{V}$ with nowhere vanishing field strength $G=\mathrm{i} \nabla^{\alpha} \bar{\nabla}_{\alpha} \mathcal{V}$. This is the case for Type II minimal supergravity [52, 78]. Then, the first term in (6.13) may be represented in the $B F$-form (6.1), specifically:

$$
\int \mathrm{d}^{3} x \mathrm{~d}^{2} \theta \mathrm{d}^{2} \bar{\theta} E \mathcal{L}=\int \mathrm{d}^{3} x \mathrm{~d}^{2} \theta \mathrm{d}^{2} \bar{\theta} E \mathcal{V} \boldsymbol{G}, \quad \boldsymbol{G}:=\mathrm{i} \nabla^{\alpha} \bar{\nabla}_{\alpha} \frac{\mathcal{L}}{G} .
$$

We see that the linear multiplet action (6.1) allows us to describe a broad class of locally supersymmetric models. However, this action principle is not universal for, in general, it cannot be used to describe the chiral term in (6.13) and its conjugated antichiral one. On the other hand, the (anti)chiral action is truly universal in $\mathcal{N}=2$ supersymmetry, due to the identity [52]

$$
\int \mathrm{d}^{3} x \mathrm{~d}^{2} \theta \mathrm{d}^{2} \bar{\theta} E \mathcal{L}=\int \mathrm{d}^{3} x \mathrm{~d}^{2} \bar{\theta} \overline{\mathcal{E}} \overline{\mathcal{L}}_{\mathrm{c}}, \quad \overline{\mathcal{L}}_{\mathrm{c}}:=-\frac{1}{4} \nabla^{\alpha} \nabla_{\alpha} \mathcal{L} .
$$

As demonstrated in [80], this action can equivalently be described in terms of a closed three-form $\Xi, \mathrm{d} \Xi=0$, such that its components

$$
\Xi=\frac{1}{2} E^{\gamma} \wedge E^{\beta} \wedge E^{a} \Xi_{a \beta \gamma}+\frac{1}{2} E^{\gamma} \wedge E^{b} \wedge E^{a} \Xi_{a b \gamma}+\frac{1}{6} E^{c} \wedge E^{b} \wedge E^{a} \Xi_{a b c}
$$

\footnotetext{
${ }^{15}$ The explicit expression for $\mathcal{E}$ in terms of the supergravity prepotentials is given in [79].
} 
are expressed via $\overline{\mathcal{L}}_{\mathrm{c}}$ as follows:

$$
\begin{aligned}
& \Xi_{a \beta \gamma}=4\left(\gamma_{a}\right)_{\beta \gamma} \overline{\mathcal{L}}_{\mathrm{c}}, \\
& \Xi_{a b \gamma}=-\mathrm{i} \varepsilon_{a b d}\left(\gamma^{d}\right)_{\gamma \delta} \bar{\nabla}^{\delta} \overline{\mathcal{L}}_{\mathrm{c}}, \\
& \Xi_{a b c}=\frac{1}{4} \varepsilon_{a b c} \bar{\nabla}_{\delta} \bar{\nabla}^{\delta} \overline{\mathcal{L}}_{\mathrm{c}} .
\end{aligned}
$$

In summary, the $\mathcal{N}=2$ linear multiplet action (6.1) is useful but not universal. As will be shown in the next section, the situation in $\mathcal{N}=3$ supersymmetry is conceptually different.

\section{Matter-coupled $\mathcal{N}=3$ supergravity}

General off-shell matter couplings in $\mathcal{N}=3$ supergravity were constructed in [52]. Given a supergravity-matter system, its dynamics can be described by a Lagrangian $\mathcal{L}^{(2)}(v)$ which is a real weight-two projective supermultiplet, ${ }^{16}$ with $v^{i}$ the homogeneous coordinates for $\mathbb{C} P^{1}$. The corresponding action is given by eq. (C.14). We assume that the dynamical supermultiplets include an Abelian vector multiplet such that its gauge invariant field strength $G^{i j}$ is nowhere vanishing, that is $G:=\sqrt{G^{i j} G_{i j}} \neq 0$. As shown in appendix C, the action functional (C.14) can be rewritten as a $B F$ term

$$
S_{\mathrm{LM}}=\frac{1}{2 \pi \mathrm{i}} \oint_{\gamma}(v, \mathrm{~d} v) \int \mathrm{d}^{3} x \mathrm{~d}^{6} \theta E C^{(-4)} \mathcal{V} \boldsymbol{G}^{(2)},
$$

where $\mathcal{V}(v)$ is the tropical prepotential for the vector multiplet, $\nabla_{\alpha}^{(2)} \mathcal{V}=0$, and

$$
\boldsymbol{G}^{(2)}(v):=\boldsymbol{G}_{i j} v^{i} v^{j}, \quad \nabla_{\alpha}^{(2)} \boldsymbol{G}^{(2)}=0 \quad \Longleftrightarrow \quad \nabla_{\alpha}^{(i j} \boldsymbol{G}^{k l)}=0
$$

is a composite real $\mathcal{O}(2)$ or linear multiplet. The explicit expression for $\boldsymbol{G}^{(2)}(v)$ in terms of the superfield Lagrangian $\mathcal{L}^{(2)}$ is given by eq. (C.20b). Different theories correspond to different choices of the composite linear multiplet $\boldsymbol{G}_{i j}$. The action (7.1a) is invariant under gauge transformations

$$
\delta \mathcal{V}=\lambda+\breve{\lambda}, \quad \nabla_{\alpha}^{(2)} \lambda=0
$$

where the gauge parameter $\lambda$ is an arbitrary weight- 0 arctic multiplet, and $\breve{\lambda}$ its smileconjugate, see [52] for more details. Eq. (7.1a) defines the $\mathcal{N}=3$ linear multiplet action.

Instead of dealing with the symmetric spinors $G^{i j}$ and $G^{i j}$, we can equivalently work with the isovectors

$$
G^{I}:=\left(\Sigma^{I}\right)_{i j} G^{i j}, \quad G^{I}:=\left(\Sigma^{I}\right)_{i j} G^{i j}
$$

where the sigma-matrices are defined by

$$
\left(\Sigma_{I}\right)_{i j}=\left(\mathbb{1}, \mathrm{i} \sigma_{1}, \mathrm{i} \sigma_{3}\right)=\left(\Sigma_{I}\right)_{j i} .
$$

\footnotetext{
${ }^{16}$ In what follows, we do not indicate explicitly the $z$-dependence of $\mathcal{N}=3$ and $\mathcal{N}=4$ superfields.
} 


\subsection{Linear multiplet action}

It turns out that the action (7.1a) may be recast in terms of a closed three-form:

$$
J=V \wedge \boldsymbol{F}-\Sigma, \quad \mathrm{d} J=0,
$$

where $\boldsymbol{F}$ is the two-form field strength associated with $G^{I}$ and $V=E^{A} V_{A}$ is the gauge one-form associated with the prepotential $\mathcal{V}$. The three-form $\Sigma=\frac{1}{3 !} E^{C} \wedge E^{B} \wedge E^{A} \Sigma_{A B C}$ is given by

$$
\begin{aligned}
\Sigma_{\alpha \beta \gamma}^{I J K}= & 0, \\
\Sigma_{a \beta \gamma}^{J K}= & 2 \mathrm{i}\left(\gamma_{a}\right)_{\beta \gamma} \operatorname{tr}\left\{\delta^{J K} G^{I} \boldsymbol{G}_{I}-G^{J} \boldsymbol{G}^{K}-\boldsymbol{G}^{J} G^{K}\right\}, \\
\Sigma_{a b \gamma}= & \varepsilon_{a b c}\left(\gamma^{c}\right)_{\gamma} \operatorname{tr}\left\{\nabla_{\delta}^{[K} G^{I]} \boldsymbol{G}_{I}+\nabla_{\delta}^{[K} \boldsymbol{G}^{I]} G_{I}-\frac{1}{3} \nabla_{\delta}^{I} G_{I} \boldsymbol{G}^{K}-\frac{1}{3} \nabla_{\delta}^{I} \boldsymbol{G}_{I} G^{K}\right\}, \\
\Sigma_{a b c}= & -\frac{\mathrm{i}}{2} \varepsilon_{a b c} \operatorname{tr}\left\{\left(\nabla_{K}^{\gamma} \nabla_{\gamma}^{K} G^{L}\right) \boldsymbol{G}_{L}+\left(\nabla_{K}^{\gamma} \nabla_{\gamma}^{K} \boldsymbol{G}^{L}\right) G_{L}+\left(\nabla_{[K}^{\gamma} G_{L]}\right)\left(\nabla_{\gamma}^{[K} \boldsymbol{G}^{L]}\right)\right. \\
& \left.-\frac{2}{9}\left(\nabla^{\gamma K} G_{K}\right)\left(\nabla_{\gamma}^{L} \boldsymbol{G}_{L}\right)\right\} .
\end{aligned}
$$

The component action generated by $J$ is

$$
\begin{aligned}
S_{\mathrm{LM}}= & \frac{1}{2} \int \mathrm{d}^{3} x e\left(\varepsilon^{a b c} v_{a} \boldsymbol{f}_{b c}-2 \mathrm{i} \chi^{\gamma} \chi_{\gamma}-\frac{\mathrm{i}}{4} \lambda^{\gamma I J} \boldsymbol{\lambda}_{\gamma I J}+g^{I} \boldsymbol{h}_{I}+\boldsymbol{g}^{I} h_{I}\right. \\
& -\frac{1}{2}\left(\gamma^{a}\right)_{\gamma \delta} \psi_{a}^{\gamma}\left(\lambda^{\delta I J} \boldsymbol{g}_{J}+\boldsymbol{\lambda}^{\delta I J} g_{J}+\mathrm{i} \chi^{\delta} \boldsymbol{g}^{I}+\mathrm{i} \chi^{\delta} g^{I}\right) \\
& \left.+\frac{\mathrm{i}}{2} \varepsilon^{a b c}\left(\gamma_{a}\right)_{\gamma \delta} \psi_{b}^{\gamma} \psi_{c L}^{\delta}\left(\delta^{K L} g^{P} \boldsymbol{g}_{P}-2 g^{K} \boldsymbol{g}^{L}\right)\right),
\end{aligned}
$$

where the component fields are defined as in section 5 . They are explicitly given by

$$
\begin{aligned}
g_{I} & =G_{I}\left|, \quad \lambda_{\alpha}{ }^{I J}=2 \nabla_{\alpha}^{[I} G^{J]}\right|, \quad \chi_{\alpha}=\frac{\mathrm{i}}{3} \nabla_{\alpha}^{I} G_{I}\left|, \quad h_{I}=-\mathrm{i} \nabla^{\gamma J} \nabla_{\gamma J} G_{I}\right|, \\
\boldsymbol{g}_{I} & =\boldsymbol{G}_{I}\left|, \quad \boldsymbol{\lambda}_{\alpha}{ }^{I J}=2 \nabla_{\alpha}^{[I} \boldsymbol{G}^{J]}\right|, \quad \chi_{\alpha}=\frac{\mathrm{i}}{3} \nabla_{\alpha}^{I} \boldsymbol{G}_{I}\left|, \quad \boldsymbol{h}_{I}=-\mathrm{i} \nabla^{\gamma J} \nabla_{\gamma J} \boldsymbol{G}_{I}\right|, \\
v_{a} & =e_{a}{ }^{m} V_{m}\left|=V_{a}\right|+\frac{1}{2} \psi_{a I}^{\alpha} V_{\alpha}^{I} \mid, \\
\boldsymbol{f}_{a b} & =\boldsymbol{F}_{a b} \mid-\frac{1}{2}\left(\psi_{[a}{ }^{K} \gamma_{b]} \boldsymbol{\lambda}_{K}\right)+\frac{\mathrm{i}}{2} \psi_{a}^{\gamma K} \psi_{b \gamma}{ }^{L} \boldsymbol{g}_{K L} \\
& =-\frac{\mathrm{i}}{12} \varepsilon_{a b c}\left(\gamma^{c}\right)^{\alpha \beta} \varepsilon^{I J K} \nabla_{\alpha I} \nabla_{\beta J} \boldsymbol{G}_{K} \mid-\frac{1}{4} \varepsilon^{I J K}\left(\psi_{[a I} \gamma_{b]} \boldsymbol{\lambda}_{J K}\right)+\frac{\mathrm{i}}{2} \varepsilon^{I J K} \psi_{a I}^{\gamma} \psi_{b \gamma J} \boldsymbol{g}_{K} .
\end{aligned}
$$

To prove that the $\mathcal{N}=3$ linear multiplet action (7.1a) has the component form (7.7), it suffices to redo, in a 3D setting, the $4 \mathrm{D} \mathcal{N}=2$ analysis given in [81].

\subsection{Composite $\mathcal{O}(2)$ multiplet}

We now present a special example of the composite $\mathcal{O}(2)$ multiplet defined by (C.20b). We consider a vector multiplet Lagrangian of the form

$$
\mathcal{L}^{(2)} \propto G^{(2)} \ln \frac{G^{(2)}}{\mathrm{i} \Upsilon^{(1)} \breve{\Upsilon}^{(1)}},
$$


where $\Upsilon^{(1)}(v)$ is a weight-1 arctic multiplet and $\breve{\Upsilon}^{(1)}(v)$ its smile-conjugated antarctic multiplet. The superfields $\Upsilon^{(1)}$ and $\breve{\Upsilon}^{(1)}$ are pure gauge degrees of freedom [52]. In the rigid supersymmetric limit, the Lagrangian (7.9) describes a superconformal vector multiplet, which is the $3 \mathrm{D} \mathcal{N}=3$ analogue of the $4 \mathrm{D} \mathcal{N}=2$ improved tensor multiplet $[82,83] .{ }^{17}$

With the Lagrangian (7.9), the contour integral in (C.20b) can be evaluated using the techniques of [85]. Alternatively, one may look for a dimension-1 primary superfield that obeys the Bianchi identity (4.15a). The resulting composite $\mathcal{O}(2)$ multiplet is

$$
G^{I}=\mathrm{i} \frac{G_{J}}{G^{2}} \nabla^{\alpha(I} \nabla_{\alpha}^{J)} G-\frac{\mathrm{i}}{4 G^{3}} G^{I} \nabla^{\alpha[J} G^{K]} \nabla_{\alpha[J} G_{K]}-\frac{\mathrm{i}}{18 G^{3}} G^{I} \nabla_{J}^{\alpha} G^{J} \nabla_{\alpha}^{K} G_{K},
$$

where

$$
G^{2}:=G^{I} G_{I}=\frac{1}{2} G^{I J} G_{I J}=G^{i j} G_{i j}
$$

is required to be nowhere vanishing. The $\mathcal{O}(2)$ multiplet may be expressed in terms of $\mathrm{SU}(2)$ indices as follows

$$
G^{i j}=\mathrm{i} \frac{G_{k l}}{G^{2}} \nabla^{\alpha i j} \nabla_{\alpha}^{k l} G-\frac{\mathrm{i}}{4 G^{3}} G^{i j} \nabla^{\alpha k p} G_{k}^{q} \nabla_{\alpha}^{l}\left(p G_{q) l}-\frac{\mathrm{i}}{18 G^{3}} G^{i j} \nabla_{k l}^{\alpha} G^{k l} \nabla_{\alpha}^{p q} G_{p q} .\right.
$$

\subsection{Supercurrent}

Before turning to a consideration of specific supergravity models, it is worth giving a few remarks concerning matter couplings to $\mathcal{N}=3$ conformal supergravity (see also $[46,50]$ ). In general, matter-coupled conformal supergravity is described by an action of the form

$$
S=\frac{1}{\widetilde{\mu}} S_{\mathrm{CSG}}+S_{\mathrm{matter}} .
$$

Here $S_{\mathrm{CSG}}$ denotes the $\mathcal{N}=3$ conformal supergravity action [45] and $S_{\text {matter }}$ the matter action. The equation of motion for the Weyl multiplet is ${ }^{18}$

$$
\frac{1}{\mu} W_{\alpha}+T_{\alpha}=0
$$

where $T_{\alpha}$ is the matter supercurrent. As a result the supercurrent $T_{\alpha}$ must have the same properties as the super Cotton tensor $W_{\alpha}$. Specifically, $T_{\alpha}$ must be a primary superfield of dimension $3 / 2$,

$$
S_{\beta}^{J} T_{\alpha}=0, \quad \mathbb{D} T_{\alpha}=\frac{3}{2} T_{\alpha},
$$

and obey the conservation equation

$$
\nabla_{I}^{\alpha} T_{\alpha}=0
$$

The latter holds provided the matter equations of motion are satisfied.

\footnotetext{
${ }^{17}$ The $4 \mathrm{D} \mathcal{N}=1$ improved tensor multiplet was introduced in [84]. The $\mathcal{N}=2$ construction of [82, 83] is a natural extension of the one given in [84].

${ }^{18}$ The coupling constants $\widetilde{\mu}$ and $\mu$ differ from each other by some numerical coefficient.
} 
Matter-coupled Poincaré or anti-de Sitter supergravities can also be described by actions of the type $(7.13)$ with $1 / \widetilde{\mu}=0$. The matter supermultiplets have to include a conformal compensator. In what follows, the latter is assumed to be the vector multiplet described by the field strength $G^{I}$. The supergravity equation of motion is

$$
T_{\alpha}^{I}=0
$$

As an example, consider $\mathcal{N}=3 \mathrm{AdS}$ supergravity. It can be described by the Lagrangian [52]

$$
\mathcal{L}_{\mathrm{SG}}^{(2)}=\frac{1}{\kappa}\left\{G^{(2)} \ln \frac{G^{(2)}}{\mathrm{i} \Upsilon^{(1)} \breve{\Upsilon}^{(1)}}+\frac{1}{2} \xi \mathcal{V} G^{(2)}\right\}
$$

with $\kappa$ and $\xi$ the gravitational and cosmological constants respectively. The cosmological term is a $\mathrm{U}(1)$ Chern-Simons term. The choice $\xi=0$ corresponds to Poincaré supergravity. The corresponding supercurrent is

$$
\kappa T_{\alpha}=\frac{\mathrm{i}}{G} \varepsilon^{I J K} G_{I} \nabla_{\alpha J} G_{K} .
$$

One can show that if $G^{I}$ satisfies the equation of motion for $\mathcal{V}, G^{I}+\xi G^{I}=0$, the supercurrent does obey eq. (7.16).

\section{$7.4(2,1)$ anti-de Sitter supergravity}

It was discovered by Achúcarro and Townsend [86] that three-dimensional $\mathcal{N}$-extended antide Sitter (AdS) supergravity exists in $[\mathcal{N} / 2]+1$ different versions, with $[\mathcal{N} / 2]$ the integer part of $\mathcal{N} / 2$. These were called the $(p, q)$ supergravity theories where the non-negative integers $p \geq q$ are such that $\mathcal{N}=p+q$.

We wish to demonstrate that the Lagrangian (7.18) describes (2,1) AdS supergravity. To see this we will degauge, following the procedure described in [50], the corresponding equations of motion,

$$
\begin{aligned}
G^{I}+\xi G^{I} & =0, \\
\kappa T_{\alpha}=\frac{\mathrm{i}}{G} \varepsilon^{I J K} G_{I} \nabla_{\alpha J} G_{K} & =0,
\end{aligned}
$$

to $\mathrm{SO}(3)$ superspace $[51,52]$. As in [50], the covariant derivatives of $\mathrm{SO}(3)$ superspace are denoted $\mathcal{D}_{A}=\left(\mathcal{D}_{a}, \mathcal{D}_{\alpha}^{I}\right)$.

Using the results of [50] we can degauge our expression for $\boldsymbol{G}^{I}$, eq. (7.10), to

$$
\begin{aligned}
G^{I}= & \mathrm{i} \frac{G_{J}}{G^{2}}\left(\mathcal{D}^{\alpha(I} \mathcal{D}_{\alpha}^{J)}-4 \mathrm{i} S^{I J}\right) G-\frac{\mathrm{i}}{4 G^{3}} G^{I} \mathcal{D}^{\alpha[J} G^{K]} \mathcal{D}_{\alpha[J} G_{K]} \\
& -\frac{\mathrm{i}}{18 G^{3}} G^{I} \mathcal{D}_{J}^{\alpha} G^{J} \mathcal{D}_{\alpha}^{K} G_{K} .
\end{aligned}
$$

The covariant derivatives $\mathcal{D}_{A}$ no longer contain the dilatation and special conformal generators,

$$
\mathcal{D}_{A}=E_{A}^{M} \partial_{M}-\frac{1}{2} \Omega_{A}^{a b} M_{a b}-\frac{1}{2} \Phi_{A}^{P Q} N_{P Q}
$$


The original local dilatation symmetry is now realized in terms of the super-Weyl transformations. In $\mathrm{SO}(3)$ superspace, there are two dimension-1 real torsion tensors, $S^{I J}=S^{J I}$ and $C_{a}{ }^{I J}=-C_{a}{ }^{J I}$. The super Cotton tensor $W_{\alpha}$ becomes a descendant of $C_{a}{ }^{I J}$,

$$
W_{\alpha}=\frac{\mathrm{i}}{12} \varepsilon_{I J K} \mathcal{D}^{\beta I} C_{\alpha \beta}{ }^{J K} .
$$

We refer the reader to [50] for more details about the degauging procedure.

Using the super-Weyl transformation of $G$ [52],

$$
G^{\prime}=e^{\sigma} G,
$$

we can impose the gauge condition

$$
G=1
$$

Taking a spinor derivative of $G$ then gives

$$
G_{K} \mathcal{D}_{\alpha}^{[I} G^{K]}+\frac{1}{3} G^{I} \mathcal{D}_{\alpha}^{K} G_{K}=0,
$$

which requires

$$
\mathcal{D}_{\alpha}^{K} G_{K}=0, \quad G_{J} \mathcal{D}_{\alpha}^{[I} G^{J]}=0 .
$$

Note that the Bianchi identity now simplifies to

$$
\mathcal{D}_{\alpha}^{I} G^{J}=\mathcal{D}_{\alpha}^{[I} G^{J]} .
$$

Since the supercurrent vanishes, eq. (7.20b), we must also have

$$
G_{[I} \mathcal{D}_{\alpha J} G_{K]}=0
$$

On the other hand, using eq. (7.26) we find

$$
\begin{aligned}
G_{I} \mathcal{D}_{\alpha J} G_{K} & =G_{[I} \mathcal{D}_{\alpha J} G_{K]}+\frac{2}{3} G_{I} \mathcal{D}_{\alpha J} G_{K}-\frac{2}{3} G_{[J} \mathcal{D}_{\alpha K]} G_{I} \\
& =\frac{2}{3} G_{I} \mathcal{D}_{\alpha J} G_{K}-\frac{2}{3} G_{[J} \mathcal{D}_{\alpha K]} G_{I}
\end{aligned}
$$

Contracting the above with $G^{I}$ and implementing eq. (7.27) tells us that $G^{I}$ is covariantly constant,

$$
\mathcal{D}_{\alpha J} G_{K}=0 .
$$

The fact that $G^{I}$ is covariantly constant strongly constrains the superspace geometry. In particular, we have

$$
\begin{aligned}
0= & \left\{\mathcal{D}_{\alpha}^{I}, \mathcal{D}_{\beta}^{J}\right\} G^{K} \\
= & 2 \mathrm{i} \delta^{I J} \mathcal{D}_{\alpha \beta} G^{K}-4 \mathrm{i} \varepsilon_{\alpha \beta} S^{K[I} G^{J]}-4 \mathrm{i} \varepsilon_{\alpha \beta} \delta^{K[I} S^{J] L} G_{L} \\
& -4 \mathrm{i} C_{\alpha \beta}{ }^{K(I} G^{J)}+4 \mathrm{i} C_{\alpha \beta}{ }^{L(I} \delta^{J) K} G_{L},
\end{aligned}
$$


which fixes the form of the curvature components as

$$
\begin{aligned}
S^{I J} & =S\left(\delta^{I J}-2 G^{I} G^{J}\right), \quad S:=S^{K}{ }_{K}, \\
C_{\alpha \beta}{ }^{I J} & =0 .
\end{aligned}
$$

The composite vector multiplet now reduces to

$$
G^{I}=-4 S G^{I} \quad \Longrightarrow \quad 4 S=\xi .
$$

Due to the equation of motion (7.20a), $S$ is seen to be constant,

$$
\mathcal{D}_{\alpha}^{I} S=0 .
$$

As a result, the covariant derivatives corresponds to $(2,1)$ AdS superspace [87]. Therefore the theory (7.18) indeed describes $(2,1)$ AdS supergravity.

Without a cosmological constant, $\xi=0$, we find

$$
S^{I J}=0
$$

and the resulting geometry corresponds to Minkowski superspace.

\subsection{Topologically massive supergravity}

Topologically massive $\mathcal{N}=3$ supergravity ${ }^{19}$ can be described by the action

$$
S_{\mathrm{TMSG}}=\frac{1}{\widetilde{\mu}} S_{\mathrm{CSG}}+S_{\mathrm{SG}}
$$

where $S_{\mathrm{SG}}$ corresponds to the supergravity Lagrangian (7.18).

The equation of motion for the Weyl multiplet now becomes

$$
T_{\alpha}+\frac{1}{\mu} W_{\alpha}=0
$$

compare with (7.20b). The equation of motion for $\mathcal{V}$ coincides with (7.20a).

We would like to degauge the equations of motion to $\mathrm{SO}(3)$ superspace. Eq. (7.38) tells us that

$$
\frac{\mathrm{i}}{\kappa G} \varepsilon^{I J K} G_{I} \mathcal{D}_{\alpha J} G_{K}=-\frac{1}{\mu} W_{\alpha},
$$

where upon degauging the Cotton tensor is

$$
W_{\alpha}=\frac{\mathrm{i}}{12} \varepsilon_{I J K} \mathcal{D}^{\beta I} C_{\alpha \beta}{ }^{J K}=\frac{\mathrm{i}}{6} \mathcal{D}^{\beta I} C_{\alpha \beta I} .
$$

Using the gauge condition $G=1$ we find

$$
G_{[I} \mathcal{D}_{\alpha J} G_{K]}=\frac{\mathrm{i}}{6 \hat{\mu}} \varepsilon_{I J K} W_{\alpha},
$$

\footnotetext{
${ }^{19}$ Topologically massive $\mathcal{N}=1$ supergravity was introduced in $[88,89]$. The off-shell versions of topologically massive $\mathcal{N}=2$ supergravity were presented in [80].
} 
where we have defined the constant

$$
\hat{\mu}=\frac{\mu}{\kappa} .
$$

Contracting with $G_{K}$ gives

$$
\mathcal{D}_{\alpha[I} G_{J]}=\frac{\mathrm{i}}{2 \hat{\mu}} \varepsilon_{I J K} G^{K} W_{\alpha} .
$$

Therefore the Bianchi identity becomes

$$
\mathcal{D}_{\alpha}^{I} G^{J}=\frac{\mathrm{i}}{2 \hat{\mu}} \varepsilon^{I J K} G_{K} W_{\alpha}=-\frac{1}{12 \hat{\mu}} \varepsilon^{I J K} G_{K} \mathcal{D}^{\beta L} C_{\alpha \beta L} .
$$

Next, we notice that on the one hand, due to eq. (7.44), we have

$$
\left\{\mathcal{D}_{\alpha}^{I}, \mathcal{D}_{\beta}^{J}\right\} G^{K}=-\frac{1}{4 \hat{\mu}^{2}} \varepsilon_{\alpha \beta} \delta^{K[I} G^{J]} W^{\gamma} W_{\gamma}-\frac{\mathrm{i}}{\hat{\mu}} G^{K(I} \mathcal{D}_{(\alpha}^{J)} W_{\beta)},
$$

while on the other we have

$$
\begin{aligned}
\left\{\mathcal{D}_{\alpha}^{I}, \mathcal{D}_{\beta}^{J}\right\} G^{K}= & 2 \mathrm{i} \delta^{I J} \mathcal{D}_{\alpha \beta} G^{K}-4 \mathrm{i} \varepsilon_{\alpha \beta} S^{K[I} G^{J]}-4 \mathrm{i} \varepsilon_{\alpha \beta} \delta^{K[I} S^{J] L} G_{L} \\
& -4 \mathrm{i} C_{\alpha \beta}{ }^{K(I} G^{J)}+4 \mathrm{i} C_{\alpha \beta}{ }^{L(I} \delta^{J) K} G_{L} .
\end{aligned}
$$

Combining the two results gives

$$
\begin{aligned}
S^{I J} & =S\left(\delta^{I J}-2 G^{I} G^{J}\right)-\frac{\mathrm{i}}{8 \hat{\mu}^{2}} W^{\gamma} W_{\gamma} G^{I} G^{J}, \quad S=S_{K}{ }^{K}, \\
\mathcal{D}_{\alpha \beta} G^{I} & =-\frac{1}{2 \hat{\mu}} G^{I J} \mathcal{D}_{(\alpha J} W_{\beta)}, \\
C_{\alpha \beta}{ }^{I J} & =\frac{1}{4 \hat{\mu}} \varepsilon^{I J K} \mathcal{D}_{(\alpha K} W_{\beta)} .
\end{aligned}
$$

These imply the equation of motion on $C_{\alpha \beta}{ }^{I}$

$$
\mathcal{D}_{(\alpha}^{I} \mathcal{D}^{\gamma J} C_{\beta) \gamma J}+24 \mathrm{i} \hat{\mu} C_{\alpha \beta}{ }^{I}=0
$$

and the corresponding equation of motion on $W_{\alpha}$

$$
\mathcal{D}_{J}^{\beta} \mathcal{D}_{\beta}^{J} W_{\alpha}+24 \mathrm{i} \hat{\mu} W_{\alpha}=0 .
$$

In addition to (7.49a), the Cotton superfield must obey the Bianchi identity

$$
\mathcal{D}^{\alpha I} W_{\alpha}=0 .
$$

Due to the conditions (7.47), the composite vector multiplet may be expressed as follows

$$
G^{I}=4 S^{I J} G_{J}+\frac{\mathrm{i}}{8 \hat{\mu}^{2}} W^{\alpha} W_{\alpha} G^{I}=-4 S G^{I}-\frac{3 \mathrm{i}}{8 \hat{\mu}^{2}} W^{\gamma} W_{\gamma} G^{I} .
$$

Furthermore, from the equation of motion (7.20a) we see that $S$ can be expressed in terms of the Cotton tensor as

$$
S=\frac{\xi}{4}-\frac{3 \mathrm{i}}{32 \hat{\mu}^{2}} W^{\alpha} W_{\alpha} .
$$


For $\xi=0$, a solution of the equations of motion for topologically massive supergravity is obtained by setting $W_{\alpha}=0$ in the above relations. This solution describes a flat superspace. Linearizing the equations (7.49) around Minkowski superspace, it may be shown that $W_{\alpha}$ obeys the Klein-Gordon equation

$$
\left(\square-m^{2}\right) W_{\alpha}=0, \quad m=4 \hat{\mu}=4 \frac{\mu}{\kappa},
$$

with $\square:=\partial^{a} \partial_{a}$. For $\xi \neq 0$, the equations of motion for topologically massive supergravity are solved by setting $W_{\alpha}=0$. Locally it describes $(2,1)$ AdS superspace [87].

\section{Matter-coupled $\mathcal{N}=4$ supergravity}

The off-shell matter couplings in $\mathcal{N}=4$ supergravity were constructed in [52]. In general, the action for a supergravity-matter system may be represented as a sum of two terms, $S=S_{\mathrm{L}}+S_{\mathrm{R}}$, the left $S_{\mathrm{L}}$ and right $S_{\mathrm{R}}$ actions, which are naturally formulated in curved $\mathcal{N}=4$ projective superspace $\mathcal{M}^{3 \mid 8} \times \mathbb{C} P_{\mathrm{L}}^{1} \times \mathbb{C} P_{\mathrm{R}}^{1}$. The left action is given by eq. (D.12), where the Lagrangian $\mathcal{L}_{\mathrm{L}}^{(2)}\left(v_{\mathrm{L}}\right)$ is a real left projective multiplet of weight two, with $v_{\mathrm{L}}=v^{i}$ the homogeneous coordinates for $\mathbb{C} P_{\mathrm{L}}^{1}$. The structure of $S_{\mathrm{R}}$ is analogous.

We assume that the dynamical supermultiplets include two Abelian vector multiplets such that their field strengths $G_{+}^{I J}$ and $G_{-}^{I J}$ are self-dual and anti-self-dual, respectively, and nowhere vanishing, $G_{ \pm}^{2}:=\frac{1}{2} G_{ \pm}^{I J} G_{ \pm I J} \neq 0$. The anti-self-dual field strength $G_{-}^{I J}$ can equivalently be realized as a left $\mathcal{O}(2)$ multiplet $G_{\mathrm{L}}\left(v_{\mathrm{L}}\right):=G_{i j} v^{i} v^{j}$. The self-dual field strength $G_{+}^{I J}$ can equivalently be realized as a right $\mathcal{O}(2)$ multiplet $G_{\mathrm{R}}\left(v_{\mathrm{R}}\right):=G_{\bar{i}} v^{\bar{i}} v^{\bar{j}}$. The vector multiplet with field strength $G_{+}^{I J}$ can be described in terms of a gauge prepotential $\mathcal{V}_{\mathrm{L}}\left(v_{\mathrm{L}}\right)$, which is a left weight-0 tropical multiplet with gauge freedom (D.16). The right $\mathcal{O}(2)$ multiplet $G_{\mathrm{R}}\left(v_{\mathrm{R}}\right)$ is constructed in terms of $\mathcal{V}_{\mathrm{L}}$ according to (D.17) and proves to be a gauge invariant field strength. Similar properties hold for the vector multiplet field strength $G_{-}^{I J}$ except all 'left' objects have to be replaced by 'right' ones and vice versa.

As demonstrated in appendix D, the left action can be recast in the $B F$ form (D.29), where $\boldsymbol{G}_{\mathrm{R}}^{(2)}\left(v_{\mathrm{R}}\right)=v_{\bar{i}} v_{\bar{j}} \boldsymbol{G}^{\bar{i} \bar{j}}$ is a composite right $\mathcal{O}(2)$ multiplet defined by (D.30). Eq. (D.29) defines the right linear multiplet action, $S_{\mathrm{RLM}}$. Obvious modifications lead to the left linear multiplet action, $S_{\mathrm{LLM}}$. One of our goals in this section is to reduce the actions $S_{\mathrm{RLM}}$ and $S_{\text {LLM }}$ to components.

\subsection{Left linear multiplet action}

The left linear multiplet action is given by

$$
S_{\mathrm{LLM}}=\frac{1}{2 \pi} \oint\left(v_{\mathrm{L}}, \mathrm{d} v_{\mathrm{L}}\right) \int \mathrm{d}^{3} x \mathrm{~d}^{8} \theta C_{\mathrm{L}}^{(-4)} \mathcal{V}_{\mathrm{L}} \boldsymbol{G}_{\mathrm{L}}^{(2)}
$$

where $\boldsymbol{G}_{\mathrm{L}}^{(2)}\left(v_{\mathrm{L}}\right)=v_{i} v_{j} \boldsymbol{G}^{i j}$ is a composite left $\mathcal{O}(2)$ multiplet, and $\mathcal{V}_{\mathrm{L}}\left(v_{\mathrm{L}}\right)$ is the tropical prepotential of the vector multiplet with field strength $G_{+}^{I J}$. The composite left $\mathcal{O}(2)$ multiplet, $\boldsymbol{G}_{\mathrm{L}}^{(2)}\left(v_{\mathrm{L}}\right)$, can be equivalently realized as the anti-self-dual $\mathrm{SO}(4)$ bivector $\boldsymbol{G}_{-}^{I J}$. 
It turns out that the action $S_{\text {LLM }}$ may be reformulated in terms of the closed three-form

$$
J=V \wedge \boldsymbol{F}-\Sigma
$$

where $\boldsymbol{F}$ is the super two-form associated with $G_{-}^{I J}$ and $V=E^{A} V_{A}$ is the gauge one-form associated with the field strength $G_{+}^{I J}$. The three-form $\Sigma=\frac{1}{3 !} E^{C} \wedge E^{B} \wedge E^{A} \Sigma_{A B C}$ is

$$
\begin{aligned}
\Sigma_{\alpha \beta \gamma}^{I J K} & =0 \\
\Sigma_{a \beta \gamma}^{J K} & =-2 \mathrm{i}\left(\gamma_{a}\right)_{\beta \gamma} G_{+}{ }^{P(J} \boldsymbol{G}_{-}{ }^{K)}{ }_{P} \\
\Sigma_{a b \gamma} K & =-\frac{1}{3} \varepsilon_{a b c}\left(\gamma^{c}\right)_{\gamma}{ }^{\delta}\left(\nabla_{\delta I} G_{+}^{I J} \boldsymbol{G}_{-J}{ }^{K}+\nabla_{\delta I} \boldsymbol{G}_{-}^{I J} G_{+J}{ }^{K}\right) \\
\Sigma_{a b c} & =\mathrm{i} \varepsilon_{a b c}\left(\frac{1}{24} \nabla_{J}^{\gamma} \nabla_{\gamma}^{I} \boldsymbol{G}_{-I K} G_{+}^{K J}+\frac{1}{24} \nabla_{J}^{\gamma} \nabla_{\gamma}^{I} G_{+I K} \boldsymbol{G}_{-}^{K J}+\frac{1}{9} \nabla_{I}^{\gamma} \boldsymbol{G}_{-}^{I J} \nabla_{\gamma}^{K} G_{+J K}\right) .
\end{aligned}
$$

The corresponding component action is

$$
\begin{aligned}
S_{\mathrm{LLM}}= & \frac{1}{2} \int \mathrm{d}^{3} x e\left(\varepsilon^{a b c} v_{a} \boldsymbol{f}_{b c}+\frac{1}{4} \hat{\boldsymbol{h}}_{+}{ }^{I J} g_{+I J}+\frac{1}{4} \hat{h}_{-}{ }^{I J} \boldsymbol{g}_{-I J}-\frac{\mathrm{i}}{2} \lambda^{\alpha I} \boldsymbol{\lambda}_{\alpha I}\right. \\
& -\frac{1}{2}\left(\gamma^{a}\right)_{\gamma \delta} \psi_{a I}^{\gamma}\left(\lambda^{\delta J} \boldsymbol{g}_{-J}{ }^{I}+\boldsymbol{\lambda}^{\delta J} g_{+J}{ }^{I}\right) \\
& \left.+\frac{\mathrm{i}}{2} \varepsilon^{a b c}\left(\gamma_{a}\right)_{\gamma \delta} \psi_{b}{ }_{K}^{\gamma} \psi_{c L}^{\delta} g_{+}{ }^{K P} \boldsymbol{g}_{-}{ }^{L}{ }_{P}\right),
\end{aligned}
$$

where the component fields are defined as in section 5 :

$$
\begin{aligned}
g_{+}^{I J} & =G_{+}^{I J}\left|, \quad \lambda_{\alpha}^{I}=\frac{2}{3} \nabla_{\alpha J} G_{+}^{I J}\right|, \quad \hat{h}_{-}{ }^{I J}=\frac{\mathrm{i}}{3} \nabla^{\gamma[I} \nabla_{\gamma K} G_{+}^{J] K} \mid-2 w g_{+}{ }^{I J}, \\
\boldsymbol{g}_{-}^{I J} & =\boldsymbol{G}_{-}^{I J}\left|, \quad \boldsymbol{\lambda}_{\alpha}^{I}=\frac{2}{3} \nabla_{\alpha J} \boldsymbol{G}_{-}^{I J}\right|, \quad \hat{\boldsymbol{h}}_{+}{ }^{I J}=\frac{\mathrm{i}}{3} \nabla^{\gamma[I} \nabla_{\gamma K} \boldsymbol{G}_{-}^{J] K} \mid+2 w \boldsymbol{g}_{-}{ }^{I J}, \\
v_{a} & =e_{a}{ }^{m} V_{m}\left|=V_{a}\right|+\frac{1}{2} \psi_{a I}{ }^{\alpha} V_{\alpha}^{I} \mid, \\
\boldsymbol{f}_{a b} & =\boldsymbol{F}_{a b} \mid-\frac{1}{2}\left(\psi_{[a}{ }^{K} \gamma_{b]} \boldsymbol{\lambda}_{K}\right)+\frac{\mathrm{i}}{2} \psi_{a}{ }^{\gamma K} \psi_{b \gamma}{ }^{L} \boldsymbol{g}_{-K L} \\
& =-\frac{\mathrm{i}}{24} \varepsilon_{a b c}\left(\gamma^{c}\right)^{\alpha \beta} \nabla_{\alpha}^{K} \nabla_{\beta}^{L} \boldsymbol{G}_{-K L} \mid-\frac{1}{2}\left(\psi_{[a}{ }^{K} \gamma_{b]} \boldsymbol{\lambda}_{K}\right)+\frac{\mathrm{i}}{2} \psi_{a}{ }^{\gamma K} \psi_{b \gamma}{ }^{L} \boldsymbol{g}_{-K L} .
\end{aligned}
$$

The component fields are defined so that $\hat{h}_{-}^{I J}$ is anti-self-dual and $\hat{\boldsymbol{h}}_{+}^{I J}$ is self-dual, see eq. (5.17b).

\subsection{Right linear multiplet action}

The right linear multiplet action is given by

$$
S_{\mathrm{RLM}}=\frac{1}{2 \pi} \oint\left(v_{\mathrm{R}}, \mathrm{d} v_{\mathrm{R}}\right) \int \mathrm{d}^{3} x \mathrm{~d}^{8} \theta C_{\mathrm{R}}^{(-4)} \mathcal{V}_{\mathrm{R}} G_{\mathrm{R}}^{(2)}
$$

where $\boldsymbol{G}_{\mathrm{R}}^{(2)}\left(v_{\mathrm{R}}\right)=v_{\bar{i}} v_{\bar{j}} \boldsymbol{G}^{\bar{i} \bar{j}}$ is a composite right $\mathcal{O}(2)$ multiplet, and $\mathcal{V}_{\mathrm{R}}\left(v_{\mathrm{R}}\right)$ is the tropical prepotential of the vector multiplet with field strength $G_{-}^{I J}$. The composite right $\mathcal{O}(2)$ multiplet, $\boldsymbol{G}_{\mathrm{R}}^{(2)}\left(v_{\mathrm{R}}\right)$, can be equivalently realized as the self-dual $\mathrm{SO}(4)$ bivector $\boldsymbol{G}_{+}^{I J}$. 
The action $S_{\text {RLM }}$ may be reformulated in terms of the closed three-form

$$
J=V \wedge \boldsymbol{F}-\Sigma,
$$

where $\boldsymbol{F}$ is the two-form field strength associated with $\boldsymbol{G}_{+}^{I J}$ and $V=E^{A} V_{A}$ is the gauge one-form associated with the field strength $G_{-}^{I J}$. The three-form $\Sigma=\frac{1}{3 !} E^{C} \wedge E^{B} \wedge E^{A} \Sigma_{A B C}$ is given by

$$
\begin{aligned}
\Sigma_{\alpha \beta \gamma}^{I J K} & =0, \\
\Sigma_{a \beta \gamma} J K & =-2 \mathrm{i}\left(\gamma_{a}\right)_{\beta \gamma} G_{-}{ }^{P(J} \boldsymbol{G}_{+}{ }^{K)} P, \\
\Sigma_{a b \gamma} K & =-\frac{1}{3} \varepsilon_{a b c}\left(\gamma^{c}\right)_{\gamma}{ }^{\delta}\left(\nabla_{\delta I} G_{-}^{I J} \boldsymbol{G}_{+}{ }^{K}+\nabla_{\delta I} \boldsymbol{G}_{+}^{I J} G_{-J}{ }^{K}\right), \\
\Sigma_{a b c} & =\mathrm{i} \varepsilon_{a b c}\left(\frac{1}{24} \nabla_{J}^{\gamma} \nabla_{\gamma}^{I} \boldsymbol{G}_{+I K} G_{-}^{K J}+\frac{1}{24} \nabla_{J}^{\gamma} \nabla_{\gamma}^{I} G_{-I K} \boldsymbol{G}_{+}^{K J}+\frac{1}{9} \nabla_{I}^{\gamma} \boldsymbol{G}_{+}^{I J} \nabla_{\gamma}^{K} G_{-J K}\right) .
\end{aligned}
$$

The corresponding component action is

$$
\begin{aligned}
S_{\mathrm{RLM}}= & \frac{1}{2} \int \mathrm{d}^{3} x e\left(\varepsilon^{a b c} v_{a} \boldsymbol{f}_{b c}+\frac{1}{4} \hat{h}_{+}{ }^{I J} \boldsymbol{g}_{+I J}+\frac{1}{4} \hat{\boldsymbol{h}}_{-}{ }^{I J} g_{-I J}-\frac{\mathrm{i}}{2} \boldsymbol{\lambda}^{\alpha I} \lambda_{\alpha I}\right. \\
& -\frac{1}{2}\left(\gamma^{a}\right)_{\gamma \delta} \psi_{a}{ }_{I}^{\gamma}\left(\boldsymbol{\lambda}^{\delta J} g_{-J}{ }^{I}+\lambda^{\delta J} \boldsymbol{g}_{+J}{ }^{I}\right) \\
& \left.+\frac{\mathrm{i}}{2} \varepsilon^{a b c}\left(\gamma_{a}\right)_{\gamma \delta} \psi_{b}{ }_{K}^{\gamma} \psi_{c L}{ }^{\delta} \boldsymbol{g}_{+}{ }^{K P}{ }_{g_{-}}{ }^{L}{ }_{P}\right),
\end{aligned}
$$

where the component fields are defined as in section 5 :

$$
\begin{aligned}
g_{-}^{I J} & =G_{-}^{I J}\left|, \quad \lambda_{\alpha}^{I}=\frac{2}{3} \nabla_{\alpha J} G_{-}^{I J}\right|, \quad \hat{h}_{+}{ }^{I J}=\frac{\mathrm{i}}{3} \nabla^{\gamma[I} \nabla_{\gamma K} G_{-}^{J] K} \mid-2 w g_{-}{ }^{I J}, \\
\boldsymbol{g}_{+}^{I J} & =\boldsymbol{G}_{+}^{I J}\left|, \quad \boldsymbol{\lambda}_{\alpha}^{I}=\frac{2}{3} \nabla_{\alpha J} \boldsymbol{G}_{+}^{I J}\right|, \quad \hat{\boldsymbol{h}}_{-}{ }^{I J}=\frac{\mathrm{i}}{3} \nabla^{\gamma[I} \nabla_{\gamma K} \boldsymbol{G}_{+}^{J] K} \mid-2 w \boldsymbol{g}_{+}{ }^{I J}, \\
v_{a} & =e_{a}{ }^{m} V_{m}\left|=V_{a}\right|+\frac{1}{2} \psi_{a I}{ }^{\alpha} V_{\alpha}^{I} \mid, \\
\boldsymbol{f}_{a b} & =\boldsymbol{F}_{a b} \mid-\frac{1}{2}\left(\psi_{[a}{ }^{K} \gamma_{b]} \boldsymbol{\lambda}_{K}\right)+\frac{\mathrm{i}}{2} \psi_{a}{ }^{\gamma K} \psi_{b \gamma}{ }^{L} \boldsymbol{g}_{+K L} \\
& =-\frac{\mathrm{i}}{24} \varepsilon_{a b c}\left(\gamma^{c}\right)^{\alpha \beta} \nabla_{\alpha}^{K} \nabla_{\beta}^{L} \boldsymbol{G}_{+K L} \mid-\frac{1}{2}\left(\psi_{[a}{ }^{K} \gamma_{b]} \boldsymbol{\lambda}_{K}\right)+\frac{\mathrm{i}}{2} \psi_{a}{ }^{\gamma K} \psi_{b \gamma}{ }^{L} \boldsymbol{g}_{+K L} .
\end{aligned}
$$

The component fields are defined so that $\hat{h}_{+}^{I J}$ is self-dual and $\hat{\boldsymbol{h}}_{-}^{I J}$ is anti-self-dual, see eq. (5.17b).

\subsection{Composite $\mathcal{O}(2)$ multiplets}

Similar to the $\mathcal{N}=3$ construction described in section 7.2 , we now present special examples of composite left and right $\mathcal{O}(2)$ multiplets. To construct $\boldsymbol{G}_{\mathrm{R}}^{(2)}$ we consider a massless vector multiplet Lagrangian of the form [52]

$$
\mathcal{L}_{\mathrm{L}}^{(2)} \propto G_{\mathrm{L}}^{(2)} \ln \frac{G_{\mathrm{L}}^{(2)}}{\mathrm{i} \Upsilon_{\mathrm{L}}^{(1)} \breve{\Upsilon}_{\mathrm{L}}^{(1)}}
$$


and make use of the representation (D.30). ${ }^{20}$ The contour integral in (D.30) may be evaluated using the technique developed in [52]. A similar analysis may be used to construct $\boldsymbol{G}_{\mathrm{L}}^{(2)}$. Alternatively, $G_{+}^{I J} \leftrightarrow \boldsymbol{G}_{\mathrm{R}}^{(2)}\left(v_{\mathrm{R}}\right)$ and $G_{-}^{I J} \leftrightarrow \boldsymbol{G}_{\mathrm{L}}^{(2)}\left(v_{\mathrm{L}}\right)$ may also be found by looking for primary superfields which obey the Bianchi identity

$$
\nabla_{\gamma}^{I} \boldsymbol{G}_{ \pm}^{J K}=\nabla_{\gamma}^{[I} \boldsymbol{G}_{ \pm}^{J K]}-\frac{2}{3} \delta^{I[J} \nabla_{\gamma L} \boldsymbol{G}_{ \pm}^{K] L}
$$

The resulting composite $\mathcal{O}(2)$ multiplets are given by

$$
\boldsymbol{G}_{ \pm}^{I J}=X_{\mp}^{I J} \pm \frac{1}{2} \varepsilon^{I J K L} X_{\mp K L}, \quad \frac{1}{2} \varepsilon_{I J K L} \boldsymbol{G}_{ \pm}^{K L}= \pm \boldsymbol{G}_{ \pm I J},
$$

where we have defined

$$
X_{ \pm}^{I J}:=\frac{\mathrm{i}}{6 G_{ \pm}} \nabla^{\gamma[I} \nabla_{\gamma K} G_{ \pm}^{J] K}+\frac{2 \mathrm{i}}{9 G_{ \pm}^{3}} \nabla^{\alpha P} G_{ \pm K P} \nabla_{\alpha Q} G_{ \pm}^{Q[I} G_{ \pm}^{J] K}
$$

To show that $\boldsymbol{G}_{ \pm}^{I J}$ is primary and satisfies the Bianchi identity, the following identities prove useful

$$
\begin{aligned}
G_{ \pm}^{I K} G_{ \pm J K} & =\frac{1}{2} \delta_{J}^{I} G_{ \pm}^{2}, \\
\varepsilon^{I J K L} G_{ \pm L P} & =\mp 3 \delta_{P}^{[I} G_{ \pm}^{J K]}
\end{aligned}
$$

In the isospinor notation, the composite $\mathcal{O}(2)$ multiplets constructed read

$$
\begin{aligned}
G^{\overline{i j}} & =\frac{\mathrm{i}}{6 G_{-}} \nabla^{\alpha i(\bar{i}} \nabla_{\alpha}^{j \bar{j})} G_{i j}-\frac{2 \mathrm{i}}{9 G_{-}^{3}} \nabla^{\alpha i(\bar{i}} G_{i j} \nabla_{\alpha}^{k \bar{j})} G_{k l} G^{j l} \\
& \left.=\frac{\mathrm{i}}{4} \nabla^{\alpha i(\bar{i}} \nabla_{\alpha}^{j \bar{j}}\right)\left(\frac{G_{i j}}{G_{-}}\right), \\
G^{i j} & =\frac{\mathrm{i}}{6 G_{+}} \nabla^{\alpha(i \bar{i}} \nabla_{\alpha}^{j) \bar{j}} G_{\bar{i} \bar{j}}-\frac{2 \mathrm{i}}{9 G_{+}^{3}} \nabla^{\alpha(i \bar{i}} G_{\bar{i} \bar{j}} \nabla_{\alpha}^{j) \bar{k}} G_{\bar{k} \bar{l}} G^{\overline{l j}} \\
& =\frac{\mathrm{i}}{4} \nabla^{\alpha(i \bar{i}} \nabla_{\alpha}^{j) \bar{j}}\left(\frac{G_{\overline{i j}}}{G_{+}}\right) .
\end{aligned}
$$

These expressions may be compared with the $4 \mathrm{D} \mathcal{N}=2$ results in [85].

For completeness we give the expressions for the composite $\mathcal{O}(2)$ multiplets in $\mathrm{SO}(4)$ superspace:

$$
\begin{aligned}
G^{\bar{i} \bar{j}} & =\frac{\mathrm{i}}{4}\left(\mathcal{D}^{\alpha i(\bar{i}} \mathcal{D}_{\alpha}^{j \bar{j})}+8 \mathrm{i} \mathcal{S}^{i j \bar{i} \bar{j}}\right)\left(\frac{G_{i j}}{G_{-}}\right), \\
G^{i j} & =\frac{\mathrm{i}}{4}\left(\mathcal{D}^{\alpha(i \bar{i}} \mathcal{D}_{\alpha}^{j) \bar{j}}+8 \mathrm{i} \mathcal{S}^{i j \bar{i} \bar{j}}\right)\left(\frac{G_{\overline{i j}}}{G_{+}}\right) .
\end{aligned}
$$

Here $\mathcal{S}^{i j \overline{i j}}=\mathcal{S}^{(i j)(\overline{i j})}$ is one of the two irreducible components of the torsion superfield $S^{i \bar{i}, j \bar{j}}:=S^{I J}\left(\tau_{I}\right)^{i \bar{i}}\left(\tau_{I}\right)^{j \bar{j}}$, defined by

$$
S^{i \bar{i}, j \bar{j}}=\mathcal{S}^{i j \bar{i} \bar{j}}+\varepsilon^{i j} \varepsilon^{\bar{i}} \mathcal{S} .
$$

\footnotetext{
${ }^{20}$ The arctic weight-1 hypermultiplet $\Upsilon_{L}^{(1)}$ and its smile conjugate $\breve{\Upsilon}_{L}^{(1)}$ in (8.11) are purely gauge degrees of freedom.
} 


\subsection{Supercurrent}

The remainder of this section is devoted to a study of specific supergravity theories. To start with, we would like to discuss the structure of the $\mathcal{N}=4$ supercurrent (see also $[46,50]$ ). Our consideration below is similar to the $\mathcal{N}=3$ analysis of section 7.3.

Consider a dynamical system describing $\mathcal{N}=4$ conformal supergravity coupled to matter supermultiplets. In general, the supergravity-matter action has the form

$$
S=\frac{1}{\widetilde{\mu}} S_{\mathrm{CSG}}+S_{\text {matter }}
$$

where $S_{\mathrm{CSG}}$ denotes the $\mathcal{N}=4$ conformal supergravity action [45] and $S_{\text {matter }}$ the matter action. The equation of motion for conformal supergravity reads

$$
\frac{1}{\mu} W+T=0
$$

where $W$ is the $\mathcal{N}=4$ Cotton superfield and $T$ is the matter supercurrent. It follows from this equation that the supercurrent must have the same properties as $W$. Specifically, $T$ must be a primary superfield of dimension 1 ,

$$
S_{\alpha}^{I} T=0, \quad \mathbb{D} T=T,
$$

and obey the conservation equation

$$
\nabla^{\alpha(I} \nabla_{\alpha}^{J)} T=\frac{1}{4} \delta^{I J} \nabla_{K}^{\alpha} \nabla_{\alpha}^{K} T
$$

Of course, the latter holds provided the matter equations of motion are satisfied.

Matter-coupled Poincaré or anti-de Sitter supergravities can also be described by an action of the type (8.18) with $1 / \widetilde{\mu}=0$. The matter supermultiplets have to include two conformal compensators. As before, these are chosen to be two Abelian vector multiplets such that their field strengths $G_{+}^{I J}$ and $G_{-}^{I J}$ are self-dual and anti-self-dual, respectively, and nowhere vanishing, $G_{ \pm}^{2}:=\frac{1}{2} G_{ \pm}^{I J} G_{ \pm I J} \neq 0$. The supergravity equation of motion is

$$
T=0 .
$$

As an example, let us consider $\mathcal{N}=4 \mathrm{AdS}$ supergravity. it can be described by two Lagrangians, left and right ones, which were chosen in [52] as

$$
\begin{aligned}
\mathcal{L}_{\mathrm{SG}, \mathrm{L}}^{(2)} & =\frac{1}{\kappa}\left\{G_{\mathrm{L}}^{(2)} \ln \frac{G_{\mathrm{L}}^{(2)}}{\mathrm{i} \Upsilon_{\mathrm{L}}^{(1)} \breve{\Upsilon}_{\mathrm{L}}^{(1)}}+\xi_{\mathrm{L}} \mathcal{V}_{\mathrm{L}} G_{\mathrm{L}}^{(2)}\right\}, \\
\mathcal{L}_{\mathrm{SG}, \mathrm{R}}^{(2)} & =\frac{1}{\kappa}\left\{G_{\mathrm{R}}^{(2)} \ln \frac{G_{\mathrm{R}}^{(2)}}{\mathrm{i} \Upsilon_{\mathrm{R}}^{(1)} \breve{\Upsilon}_{\mathrm{R}}^{(1)}}+\xi_{\mathrm{R}} \mathcal{V}_{\mathrm{R}} G_{\mathrm{R}}^{(2)}\right\} .
\end{aligned}
$$

where $\kappa$ is the gravitational coupling constant and the parameters $\xi_{\mathrm{L}}$ and $\xi_{\mathrm{R}}$ determine a cosmological constant. We recall that $G_{\mathrm{L}}^{(2)}\left(v_{\mathrm{L}}\right)$ and $G_{\mathrm{R}}^{(2)}\left(v_{\mathrm{R}}\right)$ are the gauge invariant field strengths for $\mathcal{V}_{\mathrm{R}}\left(v_{\mathrm{R}}\right)$ and $\mathcal{V}_{\mathrm{L}}\left(v_{\mathrm{L}}\right)$ respectively. The cosmological term is described by the 
left and right $B F$ terms in (8.23). It is known [52] that the action does not change if the $B F$ coupling constants are modified as

$$
\xi_{\mathrm{L}} \rightarrow \xi_{\mathrm{L}}+a, \quad \xi_{\mathrm{R}} \rightarrow \xi_{\mathrm{R}}-a,
$$

for any real constant $a$. For the action to be mirror invariant, we have to choose [52]

$$
\xi_{\mathrm{L}}=\xi_{\mathrm{R}} \equiv \xi / 2
$$

This choice will be assumed in what follows.

With the left and right Lagrangians given by (8.23), the supercurrent is

$$
\kappa T=G_{+}-G_{-} .
$$

It may be shown that the equations of motion for $\mathcal{V}_{\mathrm{L}}$ and $\mathcal{V}_{\mathrm{R}}$ are equivalent to

$$
G_{ \pm}^{I J}+\xi G_{ \pm}^{I J}=0
$$

where the composite superfields $\boldsymbol{G}_{ \pm}^{I J}$ are defined according to (8.13). Using these equations of motion, one can show that the supercurrent satisfies the conservation equation (8.21).

\section{5 (2,2) anti-de Sitter supergravity}

It turns out that the model $(8.23)$ describes the $(2,2)$ AdS supergravity. We will show this by degauging the equations of motion for the compensators, eq. (8.27), and the equation of motion for the Weyl multiplet,

$$
T=0 .
$$

Using the results of [50] we degauge $\boldsymbol{G}_{ \pm}^{I J}$ to $\mathrm{SO}(4)$ superspace

$$
\begin{aligned}
G_{ \pm}^{I J}= & \frac{\mathrm{i}}{3 G_{\mp}} \mathcal{D}^{\gamma[I} \mathcal{D}_{\gamma K} G_{\mp}^{J] K}+\frac{4}{G_{\mp}} S_{K}{ }^{[I} G_{\mp}^{J] K} \mp \frac{2}{G_{\mp}} W G_{\mp}^{I J} \\
& +\frac{2 \mathrm{i}}{9 G_{\mp}^{3}} \mathcal{D}^{\alpha P} G_{\mp K P} \mathcal{D}_{\alpha Q} G_{\mp}^{Q[I} G_{\mp}^{J] K} \\
& \pm \frac{\mathrm{i}}{9 G_{\mp}^{3}} \varepsilon^{I J R S} \mathcal{D}^{\alpha P} G_{\mp K P} \mathcal{D}_{\alpha}^{Q} G_{\mp Q R} G_{\mp S K} .
\end{aligned}
$$

We then use the super-Weyl transformations to impose the gauge condition

$$
G_{+}=1 \text {. }
$$

Taking a spinor derivative of $G_{+}$gives

$$
G_{+J K} \mathcal{D}_{\alpha}^{[I} G_{+}^{J K]}-\frac{2}{3} G_{+}^{I K} \mathcal{D}_{\alpha}^{J} G_{+K J}=0 .
$$

Then using the (anti-)self-duality condition

$$
\mathcal{D}_{\alpha}^{[I} G_{ \pm}^{J K]}=\mp \frac{1}{3} \varepsilon^{I J K L} \mathcal{D}_{\alpha}^{P} G_{ \pm L P},
$$


we find

$$
\mathcal{D}_{\alpha}^{J} G_{+I J}=0 \quad \Longrightarrow \quad \mathcal{D}_{\alpha}^{[I} G_{+}^{J K]}=0 .
$$

The above tells us that $G_{+}^{I J}$ is covariantly constant

$$
\mathcal{D}_{\alpha}^{I} G_{+}^{J K}=0 .
$$

Since the supercurrent vanishes (eq. (8.28)) we have

$$
G_{+}=G_{-}=1 \text {. }
$$

Similarly we deduce that $G_{-}^{I J}$ is covariantly constant

$$
\mathcal{D}_{\alpha}^{I} G_{-}^{J K}=0 .
$$

The covariant constancy of $G_{ \pm}{ }^{I J}$ has immediate consequences on the superspace geometry. In particular, we have

$$
\begin{aligned}
0= & \left\{\mathcal{D}_{\alpha}^{I}, \mathcal{D}_{\beta}^{J}\right\} G_{ \pm}^{K L} \\
= & 2 \mathrm{i} \delta^{I J} \mathcal{D}_{\alpha \beta} G_{ \pm}^{K L}-4 \mathrm{i} \varepsilon_{\alpha \beta} S^{K[I} G_{ \pm}^{J] L}+4 \mathrm{i} \varepsilon_{\alpha \beta} S^{L[I} G_{ \pm}^{J] K}+8 \mathrm{i} \varepsilon_{\alpha \beta} S^{P[I} \delta^{J][K} G_{ \pm P}{ }^{L]} \\
& -4 \mathrm{i} C_{\alpha \beta}{ }^{K(I} G_{ \pm}^{J) L}+4 \mathrm{i} C_{\alpha \beta}{ }^{L(I} G_{ \pm}^{J) K}+8 \mathrm{i} C_{\alpha \beta} P\left(I \delta^{J)[K} G_{ \pm P}{ }^{L]}\right. \\
& \mp 4 \mathrm{i} \varepsilon_{\alpha \beta} W \delta^{L[I} G_{ \pm}^{J] K} \pm 4 \mathrm{i} \varepsilon_{\alpha \beta} W \delta^{K[I} G_{ \pm}^{J] L}
\end{aligned}
$$

which gives

$$
\begin{aligned}
S^{K[I} G_{ \pm}^{J] L}-S^{L[I} G_{ \pm}^{J] K} & =2 S^{P[I} \delta^{J][K} G_{ \pm P}{ }^{L]} \pm W \delta^{K[I} G_{ \pm}^{J] L} \mp W \delta^{L[I} G_{ \pm}^{J] K}, \\
C_{\alpha \beta}{ }^{K(I} G_{ \pm}^{J) L}-C_{\alpha \beta}{ }^{L(I} G_{ \pm}^{J) K} & =2 C_{\alpha \beta}{ }^{P(I} \delta^{J)[K} G_{ \pm P}{ }^{L]} .
\end{aligned}
$$

The above leads to the constraints

$$
\begin{aligned}
S^{K(I} G_{ \pm}^{J)}{ }_{K} & =0, \quad S^{K}{ }_{K}= \pm 2 W=0, \\
C_{\alpha \beta}{ }^{I J} & =0 .
\end{aligned}
$$

Eq. (8.14a) and eq. (8.39a) tells us that $S^{I J}$ takes the form

$$
S^{I J}=2 S^{K L} G_{ \pm}{ }_{K} G_{ \pm}{ }^{J}{ }_{L}, \quad S_{K}{ }^{K}=0 .
$$

The composite $\mathcal{O}(2)$ multiplets now reduce to

$$
\boldsymbol{G}_{ \pm}^{I J}=4 S_{K}{ }^{[I} G_{\mp}^{J] K} .
$$

Combining the above result with the equation of motion (8.27) gives

$$
S_{K}^{I} G_{ \pm}^{J K}=-\frac{\xi}{4} G_{\mp}^{I J} .
$$

Then making use of eq. (8.14a) fixes the form of $S^{I J}$ as follows

$$
S^{I J}=-\frac{\xi}{2} G_{+}^{K(I} G_{-}{ }^{J)} K
$$


Therefore $S^{I J}$ must be covariantly constant

$$
\mathcal{D}_{\alpha}^{I} S^{J K}=0 .
$$

The above geometry corresponds to $(2,2)$ AdS superspace [87]. To see this, we rewrite $S^{I J}$ in the isospinor notation

$$
S^{i \bar{i}, j \bar{j}}=\frac{1}{2} \xi G^{i j} G^{\bar{i} \bar{j}}
$$

where

$$
G_{-}^{i \bar{i}, j \bar{j}}=-\varepsilon^{\bar{i} j} G^{i j}, \quad G_{+}^{i \bar{i}, i \bar{j}}=-\varepsilon^{i j} G^{\bar{i} \bar{j}} .
$$

As a result, the algebra of covariant derivative coincides with that for $(2,2)$ AdS superspace [87]. ${ }^{21}$

When $\xi=0$ the covariant derivative algebra corresponds to that of $\mathcal{N}=4$ Minkowski superspace.

\subsection{Topologically massive supergravity}

Topologically massive $\mathcal{N}=4$ supergravity can be described by the action

$$
S_{\mathrm{TMSG}}=\frac{1}{\widetilde{\mu}} S_{\mathrm{CSG}}+S_{\mathrm{SG}, \mathrm{L}}+S_{\mathrm{SG}, \mathrm{R}}
$$

where the left $S_{\mathrm{SG}, \mathrm{L}}$ and right $S_{\mathrm{SG}, \mathrm{R}}$ actions correspond to the supergravity Lagrangians (8.23). Now, the supercurrent is non-zero, since the equation of motion for the Weyl multiplet is

$$
T+\frac{1}{\mu} W=0 \quad \Longrightarrow \quad G_{+}=G_{-}+\frac{1}{\hat{\mu}} W,
$$

where $\hat{\mu}=\mu / \kappa$. We choose again the super-Weyl gauge condition (8.30), $G_{+}=1$. Then using the (anti-)self-duality condition (8.32) we find that $G_{+}^{I J}$ is covariantly constant

$$
\mathcal{D}_{\alpha}^{I} G_{+}^{J K}=0 .
$$

Following similar reasoning as in the last subsection, we derive the constraints

$$
C_{\alpha \beta}{ }^{I J}=0, \quad S^{I J}=2 S^{K L} G_{+}{ }^{I}{ }_{K} G_{+}{ }^{J}{ }_{L}, \quad S_{K}{ }^{K}=2 W .
$$

The anti-self-dual composite $\mathcal{O}(2)$ multiplet, $\boldsymbol{G}_{-}^{I J}$, becomes

$$
G_{-}^{I J}=4 S_{K}{ }^{[I} G_{+}^{J] K}+2 W G_{+}^{I J} .
$$

Using the equation of motion

$$
G_{-}^{I J}+\xi G_{-}^{I J}=0
$$

we find the form of $S^{I J}$ to be

$$
S^{I J}=-\frac{\xi}{2} G_{+}^{K(I} G_{-K}^{J)}+\frac{1}{2} W \delta^{I J} .
$$

\footnotetext{
${ }^{21}$ The super-Weyl gauge condition used in [87] was $G_{+}=G_{-}=2$, which differs from ours, eq. (8.35). However, this difference is irrelevant since $G_{+}=G_{-}$may be normalized whichever way we like.
} 
Taking a spinor derivative of $G_{-}$and using eq. (8.48) and the anti-self-duality condition (8.32) gives

$$
\begin{aligned}
& \mathcal{D}_{\alpha}^{J} G_{-I J}=-\frac{3}{\hat{\mu} G_{-}} G_{-I J} \mathcal{D}_{\alpha}^{J} W, \\
& \mathcal{D}_{\alpha}^{[I} G_{-}^{J K]}=-\frac{3}{\hat{\mu} G_{-}} G_{-}^{[I J} \mathcal{D}_{\alpha}^{K]} W,
\end{aligned}
$$

which, due to the Bianchi identity (8.12), lead to

$$
\mathcal{D}_{\alpha}^{I} G_{-}^{J K}=-\frac{3}{\hat{\mu} G_{-}} G_{-}^{[I J} \mathcal{D}_{\alpha}^{K]} W+\frac{2}{\hat{\mu} G_{-}} \delta^{I[J} G_{-}^{K] L} \mathcal{D}_{\alpha L} W
$$

Upon degauging to $\mathrm{SO}(4)$ superspace and using eq. (8.54), we find that the composite vector multiplet $\boldsymbol{G}_{+}^{I J}$ may be expressed as

$$
\begin{aligned}
G_{+}^{I J}= & \frac{\mathrm{i}}{2 \hat{\mu}^{2} G_{-}^{3}} G_{-}^{I J} \mathcal{D}_{K}^{\alpha} W \mathcal{D}_{\alpha}^{K} W \\
& +\frac{\mathrm{i}}{4 \hat{\mu} G_{-}^{2}} G_{-}^{I J} \mathcal{D}_{K}^{\alpha} \mathcal{D}_{\alpha}^{K} W-\frac{\xi W}{\hat{\mu}} G_{+}{ }^{I J} \\
& -\xi G_{-} G_{+}^{I J}-\frac{4 W}{G_{-}} G_{-}^{I J} .
\end{aligned}
$$

Then the equation of motion

$$
G_{+}^{I J}+\xi G_{+}^{I J}=0
$$

leads to

$$
0=\frac{\mathrm{i}}{2 \hat{\mu}^{2} G_{-}^{3}} G_{-}^{I J} \mathcal{D}_{K}^{\alpha} W \mathcal{D}_{\alpha}^{K} W+\frac{\mathrm{i}}{4 \hat{\mu} G_{-}^{2}} G_{-}^{I J} \mathcal{D}_{K}^{\alpha} \mathcal{D}_{\alpha}^{K} W-\frac{4 W}{G_{-}} G_{-}^{I J} .
$$

It follows that the equation of motion on $W$ is

$$
\mathcal{D}_{K}^{\alpha} \mathcal{D}_{\alpha}^{K} W+16 \mathrm{i} \hat{\mu} G_{-} W+\frac{3}{\hat{\mu} G_{-}} \mathcal{D}_{K}^{\alpha} W \mathcal{D}_{\alpha}^{K} W=0,
$$

with

$$
G_{-}=1-\frac{1}{\hat{\mu}} W
$$

This equation must be solved in conjunction with the Bianchi identity (A.27).

For $\xi=0$, a solution of the equations of motion for topologically massive supergravity is obtained by setting $W=0$ in the above relations. This solution describes a flat superspace. Linearizing the equation (8.59) and the Bianchi identity (A.27) around Minkowski superspace, it may be shown that $W$ obeys the Klein-Gordon equation

$$
\left(\square-m^{2}\right) W=0, \quad m=2 \hat{\mu}=2 \frac{\mu}{\kappa},
$$

with $\square:=\partial^{a} \partial_{a}$. 


\section{Concluding comments}

In this paper we have worked out the linear multiplet action principles in $\mathcal{N}=3$ and $\mathcal{N}=4$ conformal supergravities. At the component level, the $\mathcal{N}=3$ action is given by eq. (7.7), while the $\mathcal{N}=4$ action is a sum of the left and right sectors, given by eqs. (8.4) and (8.9) respectively. Using these locally supersymmetric actions, it is not difficult to construct the component off-shell actions for the $(2,1)$ and $(2,2)$ AdS supergravities and their topologically massive extensions. For instance, the composite $\mathcal{O}(2)$ multiplet, which has to be used in the action (7.7) in order to describe the $(2,1)$ AdS supergravity, proves to be $\boldsymbol{G}^{I}+\frac{1}{2} \xi G^{I}$, where $\boldsymbol{G}^{I}$ is given by eq. (7.10). The derivation of the component actions will be given elsewhere.

In superspace, the off-shell formulations for $(2,1)$ and $(2,2)$ AdS supergravities were given in [52]. The specific feature of $(2,1)$ AdS supergravity is that its conformal compensator is a vector multiplet that can be described in terms of the tropical prepotential $\mathcal{V}(v) .{ }^{22}$ The specific feature of $(2,2)$ AdS supergravity is that its conformal compensators are two vector multiplets that can be described using the left and right tropical prepotentials, $\mathcal{V}_{\mathrm{L}}\left(v_{\mathrm{L}}\right)$ and $\mathcal{V}_{\mathrm{R}}\left(v_{\mathrm{R}}\right) .{ }^{23}$ As concerns the $(3,0),(3,1)$ and $(4,0)$ AdS supergravity theories, the structure of the corresponding conformal compensators is not yet known, which is an interesting open problem.

Our procedure of constructing composite $\mathcal{O}(2)$ multiplets can be used to generate higher derivative couplings for vector multiplets, similar to the known results in $4 \mathrm{D} \mathcal{N}=$ 2 supersymmetry $[85,90] .{ }^{24}$ To illustrate the idea, let us fix $\mathcal{N}=3$ and consider the composite $\mathcal{O}(2)$ multiplet defined by (7.10). Choosing in (C.18) a composite prepotential of the form

$$
\mathcal{V}(v) \longrightarrow\left[\frac{G^{(2)}(v)}{G^{(2)}(v)}\right]^{n}, \quad n=1,2, \ldots
$$

leads to a family of composite real $\mathcal{O}(2)$ multiplets

$$
\boldsymbol{H}_{n}{ }^{(2)}(v):=\boldsymbol{H}_{n}{ }^{i j} v_{i} v_{j}=\Delta^{(4)} \oint_{\hat{\gamma}} \frac{(\hat{v}, \mathrm{~d} \hat{v})}{2 \pi(v, \hat{v})^{2}}\left[\frac{\boldsymbol{G}^{(2)}(\hat{v})}{G^{(2)}(\hat{v})}\right]^{n}, \quad \nabla_{\alpha}^{(2)} \boldsymbol{H}_{n}{ }^{(2)}=0 .
$$

Here the contour integral can be computed using the technique of [85]. Now, we have two types of composite $\mathcal{O}(2)$ multiplets, $\boldsymbol{G}^{(2)}(v)$ and $\boldsymbol{H}_{n}{ }^{(2)}(v)$, which differ by the number of spinor derivatives involved. Both of them can be used to generate new composite $\mathcal{O}(2)$ multiplets

$$
\Delta^{(4)} \oint_{\hat{\gamma}} \frac{(\hat{v}, \mathrm{~d} \hat{v})}{2 \pi(v, \hat{v})^{2}}\left[\frac{\boldsymbol{G}^{(2)}(\hat{v})}{G^{(2)}(\hat{v})}\right]^{p}\left[\frac{\boldsymbol{H}_{n}^{(2)}(\hat{v})}{G^{(2)}(\hat{v})}\right]^{q}
$$

\footnotetext{
${ }^{22}$ If $\xi=0$, the vector multiplet can be dualized into a weight- $1 / 2$ polar hypermultiplet [52].

${ }^{23}$ One of the vector multiplets can be dualized into a weight- $1 / 2$ polar hypermultiplet [52].

${ }^{24}$ For other constructions of higher derivative $4 \mathrm{D} \mathcal{N}=2$ supersymmetric invariants, see [91, 92] and references therein.
} 
with $p$ and $q$ non-negative integers. The above composite $\mathcal{O}(2)$ multiplets are expected to appear in low-energy effective actions for quantum $\mathcal{N}=3$ supersymmetric gauge theories. In general, such an affective action is given by (C.14) with a Lagrangian of the form

$$
\mathcal{L}^{(2)}=G^{(2)} \mathcal{L}\left(G^{(2)}, \boldsymbol{G}^{(2)}, \boldsymbol{H}_{n}{ }^{(2)}, \ldots\right),
$$

where $\mathcal{L}$ is a homogeneous function of degree zero.

In the $\mathcal{N}=4$ case, we need two vector multiplets, $G_{\mathrm{L}}^{(2)}\left(v_{\mathrm{L}}\right)$ and $G_{\mathrm{R}}^{(2)}\left(v_{\mathrm{R}}\right)$, in order to generate higher derivative composite $\mathcal{O}(2)$ multiplets.

In the rigid supersymmetric case, Zupnik has derived, building on the earlier work by Howe and Leeming [93], harmonic superspace formulations for the $\mathcal{N}=5$ vector multiplet and corresponding Chern-Simons actions [94, 95]. In this setting, the off-shell vector multiplet involves an infinite number of bosonic and fermionic degrees of freedom, which makes possible the construction of Chern-Simons actions.

It is known that the harmonic superspace approach is the most elaborated scheme to do supergraph calculations in off-shell theories with six and eight supercharges. It would be interesting to see how quantum corrections of the type (9.4) are generated within the background field formulation for quantum $3 \mathrm{D} \mathcal{N}=3$ super Yang-Mills theories [96].

\section{Acknowledgments}

We are grateful to Daniel Butter for comments on the manuscript. This work was supported in part by the Australian Research Council, projects DP1096372 and DP140103925. The work of JN was also supported in part by the Australian Research Council's Discovery Early Career Award (DECRA), project DE120101498.

\section{A Geometry of $\mathcal{N}$-extended conformal superspace}

Here we collect the essential details of the $\mathcal{N}$-extended superspace geometry of [50]. We refer the reader to $[50,52]$ for our conventions for $3 \mathrm{D}$ spinors.

We begin with a curved three-dimensional $\mathcal{N}$-extended superspace $\mathcal{M}^{3 \mid 2 \mathcal{N}}$ parametrized by local bosonic $\left(x^{m}\right)$ and fermionic coordinates $\left(\theta_{I}^{\mu}\right)$ :

$$
z^{M}=\left(x^{m}, \theta_{I}^{\mu}\right),
$$

where $m=0,1,2, \mu=1,2$ and $I=1, \cdots, \mathcal{N}$. The structure group is chosen to be $\operatorname{OSp}(\mathcal{N} \mid 4, \mathbb{R})$ and the covariant derivatives are postulated to have the form

$$
\nabla_{A}=E_{A}{ }^{M} \partial_{M}-\omega_{A}^{\underline{b}} X_{\underline{b}}=E_{A}{ }^{M} \partial_{M}-\frac{1}{2} \Omega_{A}{ }^{b c} M_{b c}-\frac{1}{2} \Phi_{A}{ }^{P Q} N_{P Q}-B_{A} \mathbb{D}-\mathfrak{F}_{A}{ }^{B} K_{B} .
$$

Here $E_{A}=E_{A}^{M} \partial_{M}$ is the inverse vielbein, $M_{a b}$ are the Lorentz generators, $N_{I J}$ are generators of the $\mathrm{SO}(\mathcal{N})$ group, $\mathbb{D}$ is the dilatation generator and $K_{A}=\left(K_{a}, S_{\alpha}^{I}\right)$ are the special superconformal generators. ${ }^{25}$

\footnotetext{
${ }^{25}$ As usual, we refer to $K_{a}$ as the special conformal generator and $S_{\alpha}^{I}$ as the $S$-supersymmetry generator.
} 
The Lorentz generators obey

$$
\begin{aligned}
{\left[M_{a b}, M_{c d}\right] } & =2 \eta_{c[a} M_{b] d}-2 \eta_{d[a} M_{b] c}, \\
{\left[M_{a b}, \nabla_{c}\right] } & =2 \eta_{c[a} \nabla_{b]}, \quad\left[M_{\alpha \beta}, \nabla_{\gamma}^{I}\right]=\varepsilon_{\gamma(\alpha} \nabla_{\beta)}^{I} .
\end{aligned}
$$

The $\mathrm{SO}(\mathcal{N})$ and dilatation generators obey

$$
\begin{aligned}
{\left[N_{K L}, N^{I J}\right] } & =2 \delta_{[K}^{I} N_{L]}^{J}-2 \delta_{[K}^{J} N_{L]}^{I}, & {\left[N_{K L}, \nabla_{\alpha}^{I}\right] } & =2 \delta_{[K}^{I} \nabla_{\alpha L]}, \\
{\left[\mathbb{D}, \nabla_{a}\right] } & =\nabla_{a}, & {\left[\mathbb{D}, \nabla_{\alpha}^{I}\right] } & =\frac{1}{2} \nabla_{\alpha}^{I} .
\end{aligned}
$$

The Lorentz and $\mathrm{SO}(\mathcal{N})$ generators act on the special conformal generators $K_{A}$ as

$$
\begin{aligned}
{\left[M_{a b}, K_{c}\right] } & =2 \eta_{c[a} K_{b]}, \quad\left[M_{\alpha \beta}, S_{\gamma}^{I}\right]=\varepsilon_{\gamma(\alpha} S_{\beta)}^{I}, \\
{\left[N_{K L}, S_{\alpha}^{I}\right] } & =2 \delta_{[K}^{I} S_{\alpha L]},
\end{aligned}
$$

while the dilatation generator acts on $K_{A}$ as

$$
\left[\mathbb{D}, K_{a}\right]=-K_{a}, \quad\left[\mathbb{D}, S_{\alpha}^{I}\right]=-\frac{1}{2} S_{\alpha}^{I} .
$$

Among themselves, the generators $K_{A}$ obey the algebra

$$
\left\{S_{\alpha}^{I}, S_{\beta}^{J}\right\}=2 \mathrm{i} \delta^{I J}\left(\gamma^{c}\right)_{\alpha \beta} K_{c} .
$$

Finally, the algebra of $K_{A}$ with $\nabla_{A}$ is given by

$$
\begin{aligned}
{\left[K_{a}, \nabla_{b}\right] } & =2 \eta_{a b} \mathbb{D}+2 M_{a b}, \\
{\left[K_{a}, \nabla_{\alpha}^{I}\right] } & =-\mathrm{i}\left(\gamma_{a}\right)_{\alpha}{ }^{\beta} S_{\beta}^{I}, \\
{\left[S_{\alpha}^{I}, \nabla_{a}\right] } & =\mathrm{i}\left(\gamma_{a}\right)_{\alpha}{ }^{\beta} \nabla_{\beta}^{I}, \\
\left\{S_{\alpha}^{I}, \nabla_{\beta}^{J}\right\} & =2 \varepsilon_{\alpha \beta} \delta^{I J} \mathbb{D}-2 \delta^{I J} M_{\alpha \beta}-2 \varepsilon_{\alpha \beta} N^{I J} .
\end{aligned}
$$

The covariant derivatives obey the (anti-)commutation relations of the form

$$
\begin{aligned}
{\left[\nabla_{A}, \nabla_{B}\right\}=} & -T_{A B}{ }^{C} \nabla_{C}-\frac{1}{2} R(M)_{A B}{ }^{c d} M_{c d}-\frac{1}{2} R(N)_{A B}{ }^{P Q} N_{P Q} \\
& -R(\mathbb{D})_{A B} \mathbb{D}-R(S)_{A B}{ }_{K}^{\gamma} S_{\gamma}^{K}-R(K)_{A B}{ }^{c} K_{c},
\end{aligned}
$$

where $T_{A B}{ }^{C}$ is the torsion, and $R(M)_{A B}{ }^{c d}, R(N)_{A B}{ }^{P Q}, R(\mathbb{D})_{A B}, R(S)_{A B}^{\gamma}$ and $R(K)_{A B^{c}}$ are the curvatures corresponding to the Lorentz, $\mathrm{SO}(\mathcal{N})$, dilatation, $S$-supersymmetry and special conformal boosts respectively.

The full gauge group of conformal supergravity, $\mathcal{G}$, is generated by covariant general coordinate transformations, $\delta_{\mathrm{cgct}}$, associated with a parameter $\xi^{A}$ and standard superconformal transformations, $\delta_{\mathcal{H}}$, associated with a parameter $\Lambda^{\underline{a}}$. The latter include the dilatation, Lorentz, $\mathrm{SO}(\mathcal{N})$, and special conformal (bosonic and fermionic) transformations. The covariant derivatives transform as

$$
\delta_{\mathcal{G}} \nabla_{A}=\left[\mathcal{K}, \nabla_{A}\right],
$$


where $\mathcal{K}$ denotes the first-order differential operator

$$
\mathcal{K}=\xi^{C} \nabla_{C}+\frac{1}{2} \Lambda^{a b} M_{a b}+\frac{1}{2} \Lambda^{I J} N_{I J}+\Lambda \mathbb{D}+\Lambda^{A} K_{A} .
$$

Covariant (or tensor) superfields transform as

$$
\delta_{\mathcal{G}} T=\mathcal{K} T
$$

In order to describe the Weyl multiplet of conformal supergravity, some of the components of the torsion and curvatures must be constrained. Following [50], the spinor derivative torsion and curvatures are chosen to resemble super-Yang Mills

$$
\left\{\nabla_{\alpha}^{I}, \nabla_{\beta}^{J}\right\}=-2 \mathrm{i} \varepsilon_{\alpha \beta} W^{I J},
$$

where $W^{I J}$ is some operator that takes values in the superconformal algebra, with $P_{A}$ replaced by $\nabla_{A}$. In [50] it was shown how to constrain $W^{I J}$ entirely in terms of the super Cotton tensor (for each value of $\mathcal{N}$ ). Remarkably, for all $\mathcal{N}$ the torsion tensor takes its constant flat space value, while the Lorentz and dilatation curvatures always vanish:

$$
\begin{aligned}
T^{a} & =-\mathrm{i} E^{\beta} \wedge E^{\gamma}\left(\gamma^{a}\right)_{\gamma \beta}, & T_{I}^{\alpha} & =0, \\
R(M)^{a b} & =0, & R(\mathbb{D}) & =0 .
\end{aligned}
$$

We now summarize the resulting covariant derivative algebra for all values of $\mathcal{N}$.

\section{A.1 The $\mathcal{N}=1$ case}

The $\mathcal{N}=1$ super Cotton tensor $W_{\alpha \beta \gamma}$ is a symmetric primary superfield of dimension- $5 / 2$

$$
S_{\delta} W_{\alpha \beta \gamma}=0, \quad K_{d} W_{\alpha \beta \gamma}=0, \quad \mathbb{D} W_{\alpha \beta \gamma}=\frac{5}{2} W_{\alpha \beta \gamma} .
$$

The algebra of covariant derivatives is given by

$$
\begin{aligned}
\left\{\nabla_{\alpha}, \nabla_{\beta}\right\} & =2 \mathrm{i} \nabla_{\alpha \beta}, \\
{\left[\nabla_{a}, \nabla_{\alpha}\right] } & =\frac{1}{4}\left(\gamma_{a}\right)_{\alpha}{ }^{\beta} W_{\beta \gamma \delta} K^{\gamma \delta}, \\
{\left[\nabla_{a}, \nabla_{b}\right] } & =-\frac{\mathrm{i}}{8} \varepsilon_{a b c}\left(\gamma^{c}\right)^{\alpha \beta} \nabla_{\alpha} W_{\beta \gamma \delta} K^{\gamma \delta}-\frac{1}{4} \varepsilon_{a b c}\left(\gamma^{c}\right)^{\alpha \beta} W_{\alpha \beta \gamma} S^{\gamma},
\end{aligned}
$$

The Bianchi identities imply an additional constraint on $W_{\alpha \beta \gamma}$, the vanishing of its spinor divergence,

$$
\nabla^{\alpha} W_{\alpha \beta \gamma}=0
$$

\section{A.2 The $\mathcal{N}=2$ case}

The $\mathcal{N}=2$ super Cotton tensor $W_{\alpha \beta}$ is a symmetric primary superfield of dimension-2

$$
S_{\gamma}^{I} W_{\alpha \beta}=0, \quad K_{c} W_{\alpha \beta}=0, \quad \mathbb{D} W_{\alpha \beta}=2 W_{\alpha \beta} .
$$


As in the $\mathcal{N}=1$ case, its spinor divergence vanishes,

$$
\nabla^{\alpha I} W_{\alpha \beta}=0 .
$$

The algebra of covariant derivatives is

$$
\begin{aligned}
\left\{\nabla_{\alpha}^{I}, \nabla_{\beta}^{J}\right\} & =2 \mathrm{i} \delta^{I J} \nabla_{\alpha \beta}-\mathrm{i} \varepsilon^{I J} \varepsilon_{\alpha \beta} W_{\gamma \delta} K^{\gamma \delta}, \\
{\left[\nabla_{a}, \nabla_{\beta}^{J}\right] } & =\frac{1}{2}\left(\gamma_{a}\right)_{\beta}{ }^{\gamma} \varepsilon^{J K} \nabla_{\gamma K} W^{\alpha \delta} K_{\alpha \delta}+\mathrm{i}\left(\gamma_{a}\right)_{\beta \gamma} \varepsilon^{J K} W^{\gamma \delta} S_{\delta K}, \\
{\left[\nabla_{a}, \nabla_{b}\right] } & =-\frac{\mathrm{i}}{8} \varepsilon_{a b c}\left(\gamma^{c}\right)^{\gamma \delta}\left(\varepsilon^{K L}\left(\nabla_{\gamma K} \nabla_{\delta L} W_{\alpha \beta} K^{\alpha \beta}+4 \mathrm{i} \nabla_{\gamma K} W_{\delta \beta} S_{L}^{\beta}\right)-8 W_{\gamma \delta} \mathcal{J}\right),
\end{aligned}
$$

where the $\mathrm{U}(1)$ generator $\mathcal{J}$ obeys

$$
N_{K L}=\mathrm{i} \varepsilon_{K L} \mathcal{J}, \quad \mathcal{J}=-\frac{\mathrm{i}}{2} \varepsilon^{K L} N_{K L}, \quad\left[\mathcal{J}, \nabla_{\alpha}^{I}\right]=-\mathrm{i} \varepsilon^{I J} \nabla_{\alpha J} .
$$

\section{A.3 The $\mathcal{N}=3$ case}

The $\mathcal{N}=3$ super Cotton tensor $W_{\alpha}$ is a primary superfield of dimension- $3 / 2$,

$$
S_{\beta}^{I} W_{\alpha}=0, \quad K_{b} W_{\alpha}=0, \quad \mathbb{D} W_{\alpha}=\frac{3}{2} W_{\alpha},
$$

with vanishing spinor divergence,

$$
\nabla^{\alpha I} W_{\alpha}=0
$$

The algebra of covariant derivatives is

$$
\begin{aligned}
\left\{\nabla_{\alpha}^{I}, \nabla_{\beta}^{J}\right\}= & 2 \mathrm{i} \delta^{I J} \nabla_{\alpha \beta}-2 \varepsilon_{\alpha \beta} \varepsilon^{I J L} W^{\gamma} S_{\gamma L}+\mathrm{i} \varepsilon_{\alpha \beta}\left(\gamma^{c}\right)^{\gamma \delta} \varepsilon^{I J K}\left(\nabla_{\gamma K} W_{\delta}\right) K_{c} \\
{\left[\nabla_{a}, \nabla_{\beta}^{J}\right]=} & \mathrm{i} \varepsilon^{J K L}\left(\gamma_{a}\right)_{\beta \gamma} W^{\gamma} N_{K L}+\mathrm{i} \varepsilon^{J K L}\left(\gamma_{a}\right)_{\beta \gamma}\left(\nabla_{K}^{\gamma} W^{\delta}\right) S_{\delta L} \\
& +\frac{1}{4} \varepsilon^{J K L}\left(\gamma_{a}\right)_{\beta \gamma}\left(\gamma^{c}\right)_{\delta \rho}\left(\nabla_{K}^{\gamma} \nabla_{L}^{\delta} W^{\rho}\right) K_{c} \\
{\left[\nabla_{a}, \nabla_{b}\right]=} & \varepsilon_{a b c}\left(\gamma^{c}\right)_{\alpha \beta}\left[-\frac{1}{2} \varepsilon^{I J K}\left(\nabla_{I}^{\alpha} W^{\beta}\right) N_{J K}-\frac{1}{4} \varepsilon^{I J K}\left(\nabla_{I}^{\alpha} \nabla_{J}^{\beta} W^{\gamma}\right) S_{\gamma K}\right. \\
& \left.+\frac{\mathrm{i}}{24} \varepsilon^{I J K}\left(\gamma^{d}\right)_{\gamma \delta}\left(\nabla_{I}^{\alpha} \nabla_{J}^{\beta} \nabla_{K}^{\gamma} W^{\delta}\right) K_{d}\right] .
\end{aligned}
$$

In order to define a large class of matter multiplets coupled to supergravity, it is often useful to switch to an isospinor notation using the isomorphism $\mathrm{SO}(3) \cong \mathrm{SU}(2) / \mathbb{Z}_{2}$. As usual, this is achieved by replacing any $\mathrm{SO}(3)$ vector index by a symmetric pair of $\mathrm{SU}(2)$ spinor indices, $\nabla_{\alpha}^{I} \rightarrow \nabla_{\alpha}^{i j}=\nabla_{\alpha}^{j i}$. The details of this correspondence are available in [52]. Here we only give the results essential for our discussion. Converting the indices of the $\mathrm{SO}(3)$ generator $N_{K L}$ into isospinor indices gives

$$
N_{i j, k l}=\frac{1}{2} \varepsilon_{j l} \mathcal{J}_{i k}+\frac{1}{2} \varepsilon_{i k} \mathcal{J}_{j l}, \quad N^{i j, k l}=-\frac{1}{2} \varepsilon^{j l} \mathcal{J}^{i k}-\frac{1}{2} \varepsilon^{i k} \mathcal{J}^{j l},
$$

where the $\mathrm{SU}(2)$ generator $\mathcal{J}^{k l}$ acts on the spinor covariant derivatives as

$$
\left[\mathcal{J}^{k l}, \nabla_{\alpha}^{i j}\right]=\varepsilon^{i(k} \nabla_{\alpha}^{l) j}+\varepsilon^{j(k} \nabla_{\alpha}^{l) i} .
$$


eq. (A.19a) turns into

$$
\begin{aligned}
\left\{\nabla_{\alpha}^{i j}, \nabla_{\beta}^{k l}\right\}= & -2 \mathrm{i} \varepsilon^{i(k} \varepsilon^{l) j} \nabla_{\alpha \beta}+\varepsilon_{\alpha \beta} \varepsilon^{j l} W^{\gamma} S_{\gamma}{ }^{i k}+\varepsilon_{\alpha \beta} \varepsilon^{i k} W^{\gamma} S_{\gamma}{ }^{j l} \\
& -\frac{\mathrm{i}}{2} \varepsilon_{\alpha \beta} \varepsilon^{j l}\left(\gamma^{c}\right)^{\gamma \delta}\left(\nabla_{\gamma}{ }^{i k} W_{\delta}\right) K_{c}-\frac{\mathrm{i}}{2} \varepsilon_{\alpha \beta} \varepsilon^{i k}\left(\gamma^{c}\right)^{\gamma \delta}\left(\nabla_{\gamma}{ }^{j l} W_{\delta}\right) K_{c} .
\end{aligned}
$$

We also have

$$
\left\{S_{\alpha}^{i j}, \nabla_{\beta}^{k l}\right\}=-2 \varepsilon_{\alpha \beta} \varepsilon^{i(k} \varepsilon^{l) j} \mathbb{D}+2 \varepsilon^{i(k} \varepsilon^{l) j} M_{\alpha \beta}+\varepsilon_{\alpha \beta} \varepsilon^{j l} \mathcal{J}^{i k}+\varepsilon_{\alpha \beta} \varepsilon^{i k} \mathcal{J}^{j l} .
$$

\section{A.4 The $\mathcal{N}>3$ case}

For all values of $\mathcal{N}>3$, we introduce the super Cotton tensor $W^{I J K L}$, which is a totally antisymmetric primary superfield of dimension-1,

$$
S_{\alpha}^{P} W^{I J K L}=0, \quad K_{a} W^{I J K L}=0, \quad \mathbb{D} W^{I J K L}=W^{I J K L} .
$$

The algebra of covariant derivatives is ${ }^{26}$

$$
\begin{aligned}
\left\{\nabla_{\alpha}^{I}, \nabla_{\beta}^{J}\right\}= & 2 \mathrm{i} \delta^{I J} \nabla_{\alpha \beta}+\mathrm{i} \varepsilon_{\alpha \beta} W^{I J K L} N_{K L}-\frac{\mathrm{i}}{\mathcal{N}-3} \varepsilon_{\alpha \beta}\left(\nabla_{K}^{\gamma} W^{I J K L}\right) S_{\gamma L} \\
& +\frac{1}{2(\mathcal{N}-2)(\mathcal{N}-3)} \varepsilon_{\alpha \beta}\left(\gamma^{c}\right)^{\gamma \delta}\left(\nabla_{\gamma K} \nabla_{\delta L} W^{I J K L}\right) K_{c} \\
{\left[\nabla_{a}, \nabla_{\beta}^{J}\right]=} & \frac{1}{2(\mathcal{N}-3)}\left(\gamma_{a}\right)_{\beta \gamma}\left(\nabla_{K}^{\gamma} W^{J P Q K}\right) N_{P Q} \\
& -\frac{1}{2(\mathcal{N}-2)(\mathcal{N}-3)}\left(\gamma_{a}\right)_{\beta \gamma}\left(\nabla_{L}^{\gamma} \nabla_{P}^{\delta} W^{J K L P}\right) S_{\delta K} \\
& -\frac{\mathrm{i}}{4(\mathcal{N}-1)(\mathcal{N}-2)(\mathcal{N}-3)}\left(\gamma_{a}\right)_{\beta \gamma}\left(\gamma^{c}\right)_{\delta \rho}\left(\nabla_{K}^{\gamma} \nabla_{L}^{\delta} \nabla_{P}^{\rho} W^{J K L P}\right) K_{c}, \\
{\left[\nabla_{a}, \nabla_{b}\right]=} & \frac{1}{4(\mathcal{N}-2)(\mathcal{N}-3)} \varepsilon_{a b c}\left(\gamma^{c}\right)_{\alpha \beta}\left(\mathrm{i}\left(\nabla_{I}^{\alpha} \nabla_{J}^{\beta} W^{P Q I J}\right) N_{P Q}\right. \\
& +\frac{\mathrm{i}}{\mathcal{N}-1}\left(\nabla_{I}^{\alpha} \nabla_{J}^{\beta} \nabla_{K}^{\gamma} W^{L I J K}\right) S_{\gamma L} \\
& \left.+\frac{1}{2 \mathcal{N}(\mathcal{N}-1)}\left(\gamma^{d}\right)_{\gamma \delta}\left(\nabla_{I}^{\alpha} \nabla_{J}^{\beta} \nabla_{K}^{\gamma} \nabla_{L}^{\delta} W^{I J K L}\right) K_{d}\right)
\end{aligned}
$$

where $W^{I J K L}$ satisfies the Bianchi identity

$$
\nabla_{\alpha}^{I} W^{J K L P}=\nabla_{\alpha}^{[I} W^{J K L P]}-\frac{4}{\mathcal{N}-3} \nabla_{\alpha Q} W^{Q[J K L} \delta^{P] I}
$$

For $\mathcal{N}=4$, the equation (A.26) is trivially satisfied, and instead a fundamental Bianchi identity occurs at dimension-2. Rewriting the super Cotton tensor as a scalar superfield, as in (5.5), the Bianchi identity is

$$
\nabla^{\alpha I} \nabla_{\alpha}^{J} W=\frac{1}{4} \delta^{I J} \nabla_{P}^{\alpha} \nabla_{\alpha}^{P} W
$$

We now we turn to a discussion of special features of the $\mathcal{N}=4$ case.

\footnotetext{
${ }^{26}$ The algebra for $\mathcal{N} \leq 3$ can be deduced from that for $\mathcal{N}>3[50]$.
} 


\section{A.5 The $\mathcal{N}=4$ case}

For each $\mathrm{SO}(4)$ vector $V_{I}$ we can associate a second-rank isospinor $V_{i \bar{i}}$

$$
V_{I} \leftrightarrow V_{i \bar{i}}:=\left(\tau^{I}\right)_{i \bar{i}} V_{i \bar{i}}, \quad\left(V_{i \bar{i}}\right)^{*}=V^{i \bar{i}} .
$$

The original $\mathrm{SO}(4)$ connection turns into a sum of two $\mathrm{SU}(2)$ connections

$$
\Phi_{A}=\left(\Phi_{\mathrm{L}}\right)_{A}+\left(\Phi_{\mathrm{R}}\right)_{A}, \quad\left(\Phi_{\mathrm{L}}\right)_{A}=\Phi_{A}{ }^{k l} \mathbf{L}_{k l}, \quad\left(\Phi_{\mathrm{R}}\right)_{A}=\Phi_{A}{ }^{\bar{k} \bar{l}} \mathbf{R}_{k l} .
$$

Note that

$$
N_{K L} \rightarrow N_{k \bar{k}, l \bar{l}}=\varepsilon_{\bar{k} \bar{l}} \mathbf{L}_{k l}+\varepsilon_{k l} \mathbf{R}_{\bar{k} \bar{l}} .
$$

The left and right operators act on the covariant derivatives as

$$
\left[\mathbf{L}^{k l}, \nabla_{\alpha}^{i \bar{i}}\right]=\varepsilon^{i(k} \nabla_{\alpha}^{l) \bar{i}}, \quad\left[\mathbf{R}^{k l}, \nabla_{\alpha}^{i \bar{i}}\right]=\varepsilon^{\bar{i}(\bar{k}} \nabla_{\alpha}^{i \bar{l})} .
$$

In the isospinor notation, the Bianchi identity on $W$ becomes

$$
\nabla^{\alpha i \bar{i}} \nabla_{\alpha}^{j \bar{j}} W=\frac{1}{4} \varepsilon^{i j} \varepsilon^{\overline{i j}} \nabla_{k \bar{k}}^{\alpha} \nabla_{\alpha}^{k \bar{k}} W
$$

The algebra of spinor covariant derivatives becomes

$$
\begin{aligned}
\left\{\nabla_{\alpha}^{i \bar{i}}, \nabla_{\beta}^{j \bar{j}}\right\}= & 2 \mathrm{i} \varepsilon^{i j} \varepsilon^{\bar{i} \bar{j}} \nabla_{\alpha \beta}+2 \mathrm{i} \varepsilon_{\alpha \beta} \varepsilon^{\bar{i} \bar{j}} W \mathbf{L}^{i j}-2 \mathrm{i} \varepsilon_{\alpha \beta} \varepsilon^{i j} W \mathbf{R}^{\overline{i j}} \\
& -\mathrm{i} \varepsilon_{\alpha \beta} \varepsilon^{i j} \nabla_{k}^{\gamma}{ }_{k}^{\bar{i}} W S_{\gamma}^{k \bar{j}}+\mathrm{i} \varepsilon_{\alpha \beta} \varepsilon^{\bar{i} \bar{j}} \nabla^{\gamma i}{ }_{\bar{k}} W S_{\gamma}^{j \bar{k}} \\
& +\frac{1}{4} \varepsilon_{\alpha \beta}\left(\varepsilon^{i j} \nabla_{\gamma k}{ }^{\bar{i}} \nabla_{\delta}^{k \bar{j}} W-\varepsilon^{\overline{i j}} \nabla_{\gamma}{ }^{j} \nabla_{\delta}^{i \bar{k}} W\right) K^{\gamma \delta} .
\end{aligned}
$$

Note that

$$
\left\{S_{\alpha}^{i \bar{i}}, \nabla_{\beta}^{j \bar{j}}\right\}=2 \varepsilon_{\alpha \beta} \varepsilon^{i j} \varepsilon^{\bar{i} \bar{j}} \mathbb{D}-2 \varepsilon^{i j} \varepsilon^{\bar{i} \bar{j}} M_{\alpha \beta}+2 \varepsilon_{\alpha \beta} \varepsilon^{\bar{i} \bar{j}} L^{i j}+2 \varepsilon_{\alpha \beta} \varepsilon^{i j} R^{\overline{i j}} .
$$

\section{B Supersymmetry transformations}

In this appendix we give the supersymmetry transformations of the component fields for vector multiplets with $\mathcal{N}<5$. For the supersymmetry transformations of the Weyl multiplet we refer the reader to [45]. In general there are additional auxiliary fields coming from the super Cotton tensor $W^{I J K L}$. These are defined for $\mathcal{N}>3$ as follows [45]

$$
\begin{aligned}
w_{I J K L} & :=W_{I J K L} \mid, \\
w_{\alpha}^{I J K} & :=-\frac{\mathrm{i}}{2(\mathcal{N}-3)} \nabla_{\alpha L} W^{I J K L} \mid, \\
y^{I J K L} & :=\frac{\mathrm{i}}{\mathcal{N}-3} \nabla^{\gamma\left[I^{I}\right.} \nabla_{\gamma P} W^{J K L] P} \mid, \\
X_{\alpha_{1} \cdots \alpha_{n}}{ }^{I_{1} \cdots I_{n+4}} & :=I(n) \nabla_{\left(\alpha_{1}\right.}^{\left[I_{1}\right.} \cdots \nabla_{\left.\alpha_{n}\right)}^{I_{n}} W^{\left.I_{n+1} \cdots I_{n+4}\right]} \mid,
\end{aligned}
$$

where $I(n)$ is defined by eq. (5.7). Expressions involving the component fields for lower values of $\mathcal{N}$ may be derived via the truncation procedure given in [45]. 
One readily finds the $Q$-supersymmetry and $S$-supersymmetry transformations of $v_{m}$ to be

$$
\begin{aligned}
\delta_{Q}(\xi) v_{m} & =-\frac{1}{2} \xi_{K}^{\gamma} e_{m}^{b}\left(\gamma_{b}\right)_{\gamma}{ }^{\delta} \lambda_{\delta}^{K}-\mathrm{i} \psi_{m}{ }_{J}^{\beta} \xi_{\beta K} g^{J K}, \\
\delta_{S}(\eta) v_{m} & =0 .
\end{aligned}
$$

The $S$-supersymmetry transformations of the non-gauge fields are

$$
\begin{aligned}
\delta_{S}(\eta) g^{I J} & =0 \\
\delta_{S}(\eta) \lambda_{\alpha}^{I} & =4 \eta_{\alpha J} g^{J I} \\
\delta_{S}(\eta) h^{I J} & =-2 \mathrm{i} \eta^{\alpha[I} \lambda_{\alpha}^{J]}-2 \eta_{K}^{\alpha} \chi_{\alpha}{ }^{I J K}, \\
\delta_{S}(\eta) \chi_{\alpha}{ }^{I J K} & =-6 \mathrm{i} \eta_{\alpha}^{[I} g^{J K]} .
\end{aligned}
$$

Their $Q$-supersymmetry transformations are

$$
\begin{aligned}
\delta_{Q}(\xi) g^{I J}= & -\mathrm{i} \xi_{K}^{\gamma} \chi_{\gamma}^{I J K}-\xi^{\gamma[I} \lambda_{\gamma}^{J]} \\
\delta_{Q}(\xi) \lambda_{\alpha}^{I}= & \mathrm{i} \xi_{\alpha J} h^{J I}-2 \mathrm{i} \xi_{J}^{\beta} \hat{\nabla}_{\beta \alpha} g^{J I}+2 \mathrm{i} \xi^{\beta I} \hat{F}_{\beta \alpha}, \\
\delta_{Q}(\xi) h^{I J}= & 2 \mathrm{i} \xi_{K}^{\alpha} \hat{\nabla}_{\alpha}^{\gamma} \chi_{\gamma}^{I J K}-2 \xi^{\alpha[I} \hat{\nabla}_{\alpha}^{\gamma} \lambda_{\gamma}^{J]}-8 \mathrm{i} \xi_{K}^{\alpha} w_{\alpha}{ }^{I J L} g_{L}{ }^{K} \\
& -8 \mathrm{i} \xi_{K}^{\alpha} w_{\alpha}^{P K[I} g_{P}{ }^{J]}+2 \xi_{K}^{\alpha} w^{I J K L} \lambda_{\alpha L}+4 \mathrm{i} \xi_{K}^{\alpha}\left[g^{K[I}, \lambda_{\alpha}^{J]}\right] \\
& +\mathrm{i} \xi_{K}^{\alpha}\left[g^{I J}, \lambda_{\alpha}^{K}\right] \\
\delta_{Q}(\xi) \chi_{\alpha}{ }^{I J K}= & \xi_{L}^{\beta} \chi_{\alpha \beta}{ }^{L I J K}-\frac{3}{2} \xi_{\alpha}^{[I} h^{J K]}-3 \xi^{\beta[I} \hat{\nabla}_{\beta \alpha} g^{J K]}+6 \xi_{\alpha L} w^{P L[I J} g^{K]} P \\
& +3 \mathrm{i} \xi_{\alpha L}\left[g^{L[I}, g^{J K]}\right],
\end{aligned}
$$

where we have made use of the covariant derivative

$$
\mathcal{D}_{a}=e_{a}{ }^{m} \mathcal{D}_{m}=e_{a}{ }^{m}\left(\partial_{m}-\frac{1}{2} \omega_{m}{ }^{b c} M_{b c}-\frac{1}{2} V_{m}{ }^{I J} N_{I J}-b_{m} \mathbb{D}-\mathrm{i} v_{m}\right)
$$

and defined ${ }^{27}$

$$
\begin{aligned}
\hat{\nabla}_{a} g^{I J}:= & \mathcal{D}_{a} g^{I J}+\frac{\mathrm{i}}{2} \psi_{a}{ }_{K}^{\beta} \chi_{\beta}{ }^{I J K}+\frac{1}{2} \psi_{a}^{\beta[I} \lambda_{\beta}^{J]}, \\
\hat{\nabla}_{a} \lambda_{\alpha}^{I}:= & \mathcal{D}_{a} \lambda_{\alpha}^{I}-\frac{\mathrm{i}}{2} \psi_{a \alpha J} h^{J I}+\mathrm{i} \psi_{a J}^{\beta} \hat{\boldsymbol{\nabla}}_{\alpha \beta} g^{J I}-\mathrm{i} \psi_{a}^{\beta I} \hat{F}_{\alpha \beta}-2 \phi_{a \alpha J} g^{J I}, \\
\hat{\nabla}_{a} \chi_{\alpha}{ }^{I J K}:= & \mathcal{D}_{a} \chi_{\alpha}{ }^{I J K}+\frac{1}{2} \psi_{a}{ }_{L}^{\beta} \chi_{\beta \alpha}{ }^{I J K L}+\frac{3}{4} \psi_{a \alpha}{ }^{[I} h^{J K]}+\frac{3}{2} \psi_{a}{ }^{\beta[I} \hat{\nabla}_{\beta \alpha} g^{J K]} \\
& -3 \psi_{a \alpha L} w^{P L[I J} g^{K]}{ }_{P}-\frac{3 \mathrm{i}}{2} \psi_{\alpha L}\left[g^{L I I}, g^{J K]}\right]+3 \mathrm{i} \phi_{a \alpha}{ }^{[I} g^{J K]} .
\end{aligned}
$$

In the above we have derived the supersymmetry transformations of the component fields for general $\mathcal{N}$. However, we are still missing the supersymmetry transformations of

$$
\chi_{\alpha_{1} \cdots \alpha_{n}} I_{1} \cdots I_{n+2}, \quad n>1 .
$$

These fields only appear for $\mathcal{N}>3$, while for $\mathcal{N}=4 \chi_{\alpha \beta}{ }^{I J K L}$ is composite once one imposes the (anti-)self-dual condition (4.25), see eq. (5.17b). Keeping in mind the definition of the component fields, eqs. (5.29) and (5.13), and the truncation procedure, we see that all the supersymmetry transformations for $\mathcal{N}<5$ are specified.

\footnotetext{
${ }^{27}$ The component $S$-supersymmetry connection is defined as in [45], $\phi_{a \beta}{ }^{J}:=e_{a}{ }^{m} \phi_{m \beta}{ }^{J}$.
} 


\section{Action principle in $\mathcal{N}=3$ supergravity}

As demonstrated in [52], general off-shell $\mathcal{N}=3$ supergravity-matter systems are naturally formulated in curved $\mathcal{N}=3$ projective superspace $\mathcal{M}^{3 \mid 6} \times \mathbb{C} P^{1}$ in terms of covariant projective multiplets. These multiplets were defined in [52] in $\mathrm{SO}(3)$ superspace. Here we briefly extend those definitions to $\mathcal{N}=3$ conformal superspace.

Let $v^{i} \in \mathbb{C}^{2} \backslash\{0\}$ be homogeneous coordinates for $\mathbb{C} P^{1}$. We use these variables to define a subset of spinor covariant derivatives

$$
\nabla_{\alpha}^{(2)}:=v_{i} v_{j} \nabla_{\alpha}^{i j}
$$

It follows from (A.22) that the operators $\nabla_{\alpha}^{(2)}$ anticommute with each other,

$$
\left\{\nabla_{\alpha}^{(2)}, \nabla_{\beta}^{(2)}\right\}=0 .
$$

This property allows us to define a family of constrained superfields.

By definition, a covariant projective multiplet of weight $n, Q^{(n)}(z, v)$, is a Lorentzscalar superfield on $\mathcal{M}^{3 \mid 6}$ that is holomorphic on an open domain of $\mathbb{C}^{2} \backslash\{0\}$ with respect to $v^{i}$, and is characterized by the following properties:

1. it obeys the analyticity constraint

$$
\nabla_{\alpha}^{(2)} Q^{(n)}=0
$$

2. it is a homogeneous function of $v^{i}$ of degree $n$,

$$
Q^{(n)}(c v)=c^{n} Q^{(n)}(v), \quad c \in \mathbb{C} \backslash\{0\} ;
$$

3. its $\mathrm{SU}(2)$ transformation is

$$
\delta_{\Lambda} Q^{(n)}=\frac{1}{2} \Lambda^{i j} \mathcal{J}_{i j} Q^{(n)}, \quad \Lambda^{i j} \mathcal{J}_{i j} Q^{(n)}=-\left(\Lambda^{(2)} \boldsymbol{\partial}^{(-2)}-n \Lambda^{(0)}\right) Q^{(n)} .
$$

Here we have defined

$$
\Lambda^{(2)}:=\Lambda^{i j} v_{i} v_{j}, \quad \Lambda^{(0)}:=\frac{v_{i} u_{j}}{(v, u)} \Lambda^{i j}, \quad(v, u):=v^{i} u_{i}
$$

and introduced the differential operator

$$
\partial^{(-2)}:=\frac{1}{(v, u)} u^{i} \frac{\partial}{\partial v^{i}} .
$$

These relations involve a fixed isospinor $u_{i}$ which is subject to the condition $(v, u) \neq 0$, but otherwise completely arbitrary. For the covariant projective multiplet, one can define the operation of smile conjugation which takes $Q^{(n)}(v)$ to its smile-conjugate $\breve{Q}^{(n)}(v)$, which is also a covariant weight- $n$ projective multiplet, see [52] for the details. Its property is

$$
\breve{Q}^{(n)}(v)=(-1)^{n} Q^{(n)}(v) .
$$

Therefore, if $n$ is even, one can define real projective multiplets, $\breve{Q}^{(2 n)}=Q^{(2 n)}$. 
A weight- $n$ isotwistor superfield $U^{(n)}(z, v)$ is defined to share with $Q^{(n)}(z, v)$ all its properties except the analyticity constraint (C.3).

In this paper, all covariant projective multiplets are assumed to be primary,

$$
S_{\alpha}^{i j} Q^{(n)}=0, \quad K_{a} Q^{(n)}=0,
$$

and hence $\left\{S_{\alpha}^{i j}, \nabla^{(2)}\right\} Q^{(n)}=0$. Then it follows from (A.23) that the dimension of $Q^{(n)}$ is equal to $n / 2$,

$$
\mathbb{D} Q^{(n)}=\frac{n}{2} Q^{(n)}
$$

An important example of covariant projective multiplets is a real $\mathcal{O}(2 n)$ multiplet, with $n=1,2, \ldots$ It is described by a real weight- $2 n$ projective superfield $H^{(2 n)}(v)$ of the form:

$$
H^{(2 n)}(v)=H^{i_{1} \ldots i_{2 n}} v_{i_{1}} \ldots v_{i_{2 n}}=\breve{H}^{(2 n)}(v) .
$$

The analyticity constraint (C.3) is equivalent to

$$
\nabla_{\alpha}^{(i j} H^{\left.k_{1} \ldots k_{2 n}\right)}=0
$$

while the reality condition $\breve{H}^{(2 n)}=H^{(2 n)}$ is equivalent to

$$
\overline{H^{i_{1} \ldots i_{2 n}}}=H_{i_{1} \ldots i_{2 n}}=\varepsilon_{i_{1} j_{1}} \cdots \varepsilon_{i_{2 n} j_{2 n}} H^{j_{1} \ldots j_{2 n}} .
$$

The field strength of an Abelian vector multiplet, $G^{(2)}$, is a real $\mathcal{O}(2)$ multiplet.

To describe the dynamics of a supergravity-matter system, one has to specify a Lagrangian, $\mathcal{L}^{(2)}(v)$, which is postulated to be a real weight-two projective multiplet. Associated with $\mathcal{L}^{(2)}$ is the supersymmetric action [52]

$$
S=\frac{1}{2 \pi \mathrm{i}} \oint_{\gamma}(v, \mathrm{~d} v) \int \mathrm{d}^{3} x \mathrm{~d}^{6} \theta E C^{(-4)} \mathcal{L}^{(2)}, \quad E^{-1}=\operatorname{Ber}\left(E_{A}{ }^{M}\right) .
$$

Here the model-independent isotwistor superfield $C^{(-4)}(v)$ of weight -4 is required to be conformally primary and of dimension -1 ,

$$
S_{\alpha}^{i j} C^{(-4)}=0, \quad K_{a} C^{(-4)}=0, \quad \mathbb{D} C^{(-4)}=-C^{(-4)},
$$

and obey the condition

$$
\Delta^{(4)} C^{(-4)}=1
$$

where

$$
\Delta^{(4)}:=\frac{\mathrm{i}}{4} \nabla^{(2) \alpha} \nabla_{\alpha}^{(2)} .
$$

As shown in [52], the action (C.14) does not change under an arbitrary infinitesimal variation of $C^{(-4)}$, and thus (C.14) is actually independent of $C^{(-4)}$. 
The second-order operator (C.17) allows us to engineer covariant projective multiplets. The point is that the superfield $\Delta^{(4)} U^{(n-4)}(v)$ is a covariant weight- $n$ projective multiplet, for any primary isotwistor superfield $U^{(n-4)}$ of dimension $n / 2-1$.

We now derive a new representation for the action (C.14) that is valid under the assumption that there is an Abelian vector multiplet such that its gauge invariant field strength $G^{i j}$ is nowhere vanishing, $G:=\sqrt{G^{i j} G_{i j}} \neq 0$. Let $\mathcal{V}(v)$ be the tropical prepotential for this multiplet. By definition, $\mathcal{V}(v)$ is a real weight-zero projective multiplet. The superfield $G^{i j}$ is a real $\mathcal{O}(2)$ multiplet which is related to $\mathcal{V}(v)$ as follows:

$$
G^{(2)}(v):=G_{i j} v^{i} v^{j}=\Delta^{(4)} \oint_{\hat{\gamma}} \frac{(\hat{v}, \mathrm{~d} \hat{v})}{2 \pi(v, \hat{v})^{2}} \mathcal{V}(\hat{v})
$$

The right-hand side on (C.18) is invariant under the gauge transformations (7.2).

In the action (C.14), we first replace $\mathcal{L}^{(2)} \rightarrow G^{(2)}\left[\mathcal{L}^{(2)} / G^{(2)}\right]$ and make use of the representation (C.18) for the first multiplier. As a next step, we can integrate by parts in order to let $\Delta^{(4)}$ hit $C^{(-4)}$ and then use (C.16). Finally, we can change the order of contour integrations to result in

$$
S=\frac{1}{2 \pi \mathrm{i}} \oint_{\hat{\gamma}}(\hat{v}, \mathrm{~d} \hat{v}) \int \mathrm{d}^{3} x \mathrm{~d}^{6} \theta E \mathcal{V}(\hat{v}) \oint_{\gamma} \frac{(v, \mathrm{~d} v)}{2 \pi(v, \hat{v})^{2}} \frac{\mathcal{L}^{(2)}(v)}{G^{(2)}(v)} .
$$

In this functional, we first re-label $v \leftrightarrow \hat{v}$, then insert the unity $1=\Delta^{(4)} C^{(-4)}(v)$ and finally integrate $\Delta^{(4)}$ by parts. Since $\mathcal{V}(v)$ obeys the constraint (C.3), the projection operator $\Delta^{(4)}$ commutes with $\mathcal{V}$, and we end up with the representation

$$
S=\frac{1}{2 \pi \mathrm{i}} \oint_{\gamma}(v, \mathrm{~d} v) \int \mathrm{d}^{3} x \mathrm{~d}^{6} \theta E C^{(-4)} \mathcal{V} \boldsymbol{G}^{(2)},
$$

where

$$
\boldsymbol{G}^{(2)}(v):=\boldsymbol{G}_{i j} v^{i} v^{j}=\Delta^{(4)} \oint_{\hat{\gamma}} \frac{(\hat{v}, \mathrm{~d} \hat{v})}{2 \pi(v, \hat{v})^{2}} \frac{\mathcal{L}^{(2)}(\hat{v})}{G^{(2)}(\hat{v})}, \quad \nabla_{\alpha}^{(2)} \boldsymbol{G}^{(2)}=0
$$

is a composite real $\mathcal{O}(2)$ multiplet. Eq. (C.20) is our new representation for the action (C.14). It is the main result of this section.

We conclude with an example that provides evidence of the universality of the projective superspace action (C.14). Let us consider the conventional $\mathcal{N}=3$ locally supersymmetric action

$$
S=\mathrm{i} \int \mathrm{d}^{3} x \mathrm{~d}^{6} \theta E \mathcal{L}, \quad \mathbb{D} \mathcal{L}=0,
$$

where the Lagrangian $\mathcal{L}$ is a dimensionless primary scalar superfield. It turns out that this action can be recast in the form (C.14) if we define

$$
\mathcal{L}^{(2)}=2 \Delta^{(4)} \frac{G \mathcal{L}}{G^{(2)}} .
$$

This may be proved using the the contour integration techniques of [85]. 


\section{Action principle in $\mathcal{N}=4$ supergravity}

Within the approach [52], off-shell $\mathcal{N}=4$ supergravity-matter systems are formulated in curved $\mathcal{N}=4$ projective superspace $\mathcal{M}^{3 \mid 8} \times \mathbb{C} P_{\mathrm{L}}^{1} \times \mathbb{C} P_{\mathrm{R}}^{1}$ in terms of covariant projective multiplets. These multiplets were defined in [52] in $\mathrm{SO}(4)$ superspace. Here we briefly extend those definitions to $\mathcal{N}=4$ conformal superspace. Our presentation is similar to the $\mathcal{N}=3$ story of the previous section.

Let $v_{\mathrm{L}}:=v^{i} \in \mathbb{C}^{2} \backslash\{0\}$ and $v_{\mathrm{R}}:=v^{\bar{i}} \in \mathbb{C}^{2} \backslash\{0\}$ be homogeneous coordinates for $\mathbb{C} P_{\mathrm{L}}^{1}$ and $\mathbb{C} P_{\mathrm{R}}^{1}$ respectively. We use these variables to define two different subsets, $\nabla_{\alpha}^{(1) \bar{i}}$ and $\nabla_{\alpha}^{(\overline{1}) i}$, in the set of spinor covariant derivatives $\nabla_{\alpha}^{i \bar{i}}$,

$$
\nabla_{\alpha}^{(1) \bar{i}}:=v_{i} \nabla_{\alpha}^{i \bar{i}}, \quad \nabla_{\alpha}^{(\overline{1}) i}:=v_{\bar{i}} \nabla_{\alpha}^{i \bar{i}} .
$$

It follows from (A.33) that the operators $\nabla_{\alpha}^{(1) \bar{i}}$ obey the anti-commutation relations:

$$
\begin{aligned}
\left\{\nabla_{\alpha}^{(1) \bar{i}}, \nabla_{\beta}^{(1) \bar{j}}\right\}= & 2 \mathrm{i} \varepsilon_{\alpha \beta} \varepsilon^{\overline{i j}} W \mathbf{L}^{(2)}+\mathrm{i} \varepsilon_{\alpha \beta} \varepsilon^{\overline{i j}} \nabla^{\gamma(1)}{ }_{\bar{k}} W S_{\gamma}^{(1) \bar{k}} \\
& -\frac{1}{4} \varepsilon_{\alpha \beta} \varepsilon^{\bar{i} \bar{j}} \nabla_{\gamma}{ }^{(1)}{ }_{\bar{k}} \nabla_{\delta}^{(1) \bar{k}} W K^{\gamma \delta} .
\end{aligned}
$$

There are two types of covariant projective multiplets, the left and right ones. A left projective multiplet of weight $n, Q_{\mathrm{L}}^{(n)}\left(v_{\mathrm{L}}\right)$, is defined to obey the constraint

$$
\nabla_{\alpha}^{(1) \bar{i}} Q_{\mathrm{L}}^{(n)}=0
$$

and is required to be a holomorphic and homogeneous function of $v_{\mathrm{L}}$ of degree $n$,

$$
Q_{\mathrm{L}}^{(n)}\left(c v_{\mathrm{L}}\right)=c^{n} Q_{\mathrm{L}}^{(n)}\left(v_{\mathrm{L}}\right), \quad c \in \mathbb{C} \backslash\{0\},
$$

on some open domain of $\mathbb{C}^{2} \backslash\{0\}$. The left projective multiplet is inert with respect to $\mathrm{SU}(2)_{\mathrm{R}}$ and transforms under $\mathrm{SU}(2)_{\mathrm{L}}$ as

$$
\begin{aligned}
\delta_{\Lambda} Q_{\mathrm{L}}^{(n)} & =\Lambda^{i j} \mathbf{L}_{i j} Q_{\mathrm{L}}^{(n)}, \\
\mathbf{L}_{i j} Q_{\mathrm{L}}^{(n)} & =-\left(\Lambda_{\mathrm{L}}^{(2)} \boldsymbol{\partial}_{\mathrm{L}}^{(-2)}-n \Lambda_{\mathrm{L}}^{(0)}\right) Q_{\mathrm{L}}^{(n)},
\end{aligned}
$$

where we have defined

$$
\Lambda_{\mathrm{L}}^{(2)}:=\Lambda^{i j} v_{i} v_{j}, \quad \Lambda_{\mathrm{L}}^{(0)}=\frac{v_{i} u_{j}}{\left(v_{\mathrm{L}}, u_{\mathrm{L}}\right)} \Lambda^{i j}
$$

and introduced the differential operator

$$
\boldsymbol{\partial}_{\mathrm{L}}^{(-2)}=\frac{1}{\left(v_{\mathrm{L}}, u_{\mathrm{L}}\right)} u^{i} \frac{\partial}{\partial v^{i}}, \quad\left(v_{\mathrm{L}}, u_{\mathrm{L}}\right)=v^{i} u_{i}
$$

The right projective multiplets are defined similarly.

In $\mathcal{N}=4$ conformal superspace, we can also introduce hybrid projective multiplets and isotwistor projective multiplets. The corresponding definitions are completely analogous to those given in [52]. 
All left and right projective multiplets are assumed to be primary, in particular

$$
S_{\alpha}^{i \bar{i}} Q_{\mathrm{L}}^{(n)}=0, \quad K_{a} Q_{\mathrm{L}}^{(n)}=0 .
$$

Hence we have the condition

$$
\left\{S_{\alpha}^{i \bar{i}}, \nabla_{\beta}^{(1) \bar{j}}\right\} Q_{\mathrm{L}}^{(n)}=0,
$$

which fixes the dimension of $Q_{\mathrm{L}}^{(n)}$

$$
\mathbb{D} Q_{\mathrm{L}}^{(n)}=\frac{n}{2} Q_{\mathrm{L}}^{(n)}
$$

In general, the $\mathcal{N}=4$ supersymmetric action may be represented as a sum of two terms, the left $S_{\mathrm{L}}$ and right $S_{\mathrm{R}}$ ones, ${ }^{28}$

$$
S=S_{\mathrm{L}}+S_{\mathrm{R}}
$$

The left action has the form

$$
S_{\mathrm{L}}=\frac{1}{2 \pi} \oint_{\gamma_{\mathrm{L}}}\left(v_{\mathrm{L}}, \mathrm{d} v_{\mathrm{L}}\right) \int \mathrm{d}^{3} x \mathrm{~d}^{8} \theta E C_{\mathrm{L}}^{(-4)} \mathcal{L}_{\mathrm{L}}^{(2)}, \quad E^{-1}=\operatorname{Ber}\left(E_{A}{ }^{M}\right),
$$

where the Lagrangian $\mathcal{L}_{\mathrm{L}}^{(2)}\left(v_{\mathrm{L}}\right)$ is a real left projective multiplet of weight 2. The action involves a model-independent primary isotwistor superfield $C_{\mathrm{L}}^{(-4)}\left(v_{\mathrm{L}}\right)$ of dimension -2 , $\mathbb{D} C_{\mathrm{L}}^{(-4)}=-2 C_{\mathrm{L}}^{(-4)}$. It is defined to be real with respect to the smile-conjugation and obey the differential equation

$$
\Delta_{\mathrm{L}}^{(4)} C_{\mathrm{L}}^{(-4)}=1
$$

Here $\Delta_{\mathrm{L}}^{(4)}$ denotes the following fourth-order operator ${ }^{29}$

$$
\Delta_{\mathrm{L}}^{(4)}=\frac{1}{96}\left(\nabla^{(2) \bar{i} \bar{j}} \nabla_{\bar{i} \bar{j}}^{(2)}-\nabla^{(2) \alpha \beta} \nabla_{\alpha \beta}^{(2)}\right)=\frac{1}{48} \nabla^{(2) \bar{i} \bar{j}} \nabla_{\bar{i} \bar{j}}^{(2)},
$$

with

$$
\nabla_{\bar{i} \bar{j}}^{(2)}:=\nabla_{(\bar{i}}^{(1) \gamma} \nabla_{\gamma \bar{j})}^{(1)}, \quad \nabla_{\alpha \beta}^{(2)}:=\nabla_{(\alpha}^{(1) \bar{k}} \nabla_{\beta) \bar{k}}^{(1)}
$$

The action (D.12) is independent of $C_{\mathrm{L}}^{(-4)}$ in the sense that it does not change under an arbitrary infinitesimal variation of $C_{\mathrm{L}}^{(-4)}$.

An Abelian vector multiplet with self-dual field strength $G_{+}^{I J}$ can be described by a left tropical prepotential $\mathcal{V}_{\mathrm{L}}\left(v_{\mathrm{L}}\right)$ defined modulo gauge transformations

$$
\delta V_{\mathrm{L}}=\lambda_{\mathrm{L}}+\breve{\lambda}_{\mathrm{L}},
$$

\footnotetext{
${ }^{28}$ There exist different action principles, in particular the one with a hybrid Lagrangian [52]. However, they may be always reduced to the form (D.11).

${ }^{29}$ The operator $\Delta_{\mathrm{L}}^{(4)}$ is a covariant projection operator. Given a covariant left projective multiplet $Q_{\mathrm{L}}^{(n)}\left(v_{\mathrm{L}}\right)$ of weight $n$, it may be represented in the form $Q_{\mathrm{L}}^{(n)}=\Delta_{\mathrm{L}}^{(4)} T_{\mathrm{L}}^{(n-4)}$, for some left isotwistor superfield $T_{\mathrm{L}}^{(n-4)}\left(v_{\mathrm{L}}\right)$, see [52] for the details.
} 
where the gauge parameter $\lambda_{\mathrm{L}}$ is an arbitrary left arctic multiplet of weight zero. The corresponding gauge invariant field strength, $G^{\bar{i} \bar{j}}$, is a right $\mathcal{O}(2)$ multiplet related to $\mathcal{V}_{\mathrm{L}}$ as follows:

$$
G_{\mathrm{R}}^{(2)}\left(v_{\mathrm{R}}\right)=v_{\bar{i}} v_{\bar{j}} G^{\bar{i} \bar{j}}=\frac{\mathrm{i}}{4} v_{\bar{i}} v_{\bar{j}} \oint \frac{\left(v_{\mathrm{L}}, \mathrm{d} v_{\mathrm{L}}\right)}{2 \pi} \frac{u_{i} u_{j}}{\left(v_{\mathrm{L}}, u_{\mathrm{L}}\right)^{2}} \nabla^{\alpha i \bar{i}} \nabla_{\alpha}^{j \bar{j}} \mathcal{V}_{\mathrm{L}}\left(v_{\mathrm{L}}\right)
$$

Here $u_{\mathrm{L}}=u^{i}$ is a constant isospinor such that $\left(v_{\mathrm{L}}, u_{\mathrm{L}}\right) \neq 0$ along the closed integration contour. One may show that the right-hand side of (D.17) is independent of $u_{\mathrm{L}}$.

The left $\mathcal{O}(2)$ multiplet $G^{i j}$ is associated with a right tropical prepotential $\mathcal{V}_{\mathrm{R}}\left(v_{\mathrm{R}}\right)$ according to the rule:

$$
G_{\mathrm{L}}^{(2)}\left(v_{\mathrm{L}}\right)=v_{i} v_{j} G^{i j}=\frac{\mathrm{i}}{4} v_{i} v_{j} \oint \frac{\left(v_{\mathrm{R}}, \mathrm{d} v_{\mathrm{R}}\right)}{2 \pi} \frac{u_{\bar{i}} u_{\bar{j}}}{\left(v_{\mathrm{R}}, u_{\mathrm{R}}\right)^{2}} \nabla^{\alpha i \bar{i}} \nabla_{\alpha}^{j \bar{j}} \mathcal{V}_{\mathrm{R}}\left(v_{\mathrm{R}}\right) .
$$

The prepotential $\mathcal{V}_{\mathrm{R}}$ can always be represented as

$$
\mathcal{V}_{\mathrm{R}}\left(v_{\mathrm{R}}\right)=\Delta_{\mathrm{R}}^{(4)} T_{\mathrm{R}}^{(-4)}\left(v_{\mathrm{R}}\right),
$$

for some isotwistor superfield $T_{\mathrm{R}}^{(-4)}\left(v_{\mathrm{R}}\right) \cdot{ }^{30}$ Here $\Delta_{\mathrm{R}}^{(4)}$ is defined similar to $\Delta_{\mathrm{L}}^{(4)}$,

$$
\Delta_{\mathrm{R}}^{(4)}=\frac{1}{48} \nabla^{(2) i j} \nabla_{i j}^{(2)}, \quad \nabla_{i j}^{(2)}:=\nabla_{(i}^{\gamma(\overline{1})} \nabla_{\gamma j)}^{(\overline{1})} .
$$

There exists an isotwistor superfield $T^{(-2,-4)}\left(\hat{v}_{\mathrm{L}}, v_{\mathrm{R}}\right)$ such that ${ }^{31}$

$$
T_{\mathrm{R}}^{(-4)}\left(v_{\mathrm{R}}\right)=\oint_{\hat{\gamma}_{\mathrm{L}}} \frac{\left(\hat{v}_{\mathrm{L}}, \mathrm{d} \hat{v}_{\mathrm{L}}\right)}{2 \pi} T^{(-2,-4)}\left(\hat{v}_{\mathrm{L}}, v_{\mathrm{R}}\right)
$$

Then, the field strength (D.18) can be rewritten in the form [52]

$$
G_{\mathrm{L}}^{(2)}\left(v_{\mathrm{L}}\right)=\Delta_{\mathrm{L}}^{(4)} \oint_{\gamma_{\mathrm{R}}} \frac{\left(v_{\mathrm{R}}, \mathrm{d} v_{\mathrm{R}}\right)}{2 \pi} \oint_{\hat{\gamma}_{\mathrm{L}}} \frac{\left(\hat{v}_{\mathrm{L}}, \mathrm{d} \hat{v}_{\mathrm{L}}\right)}{2 \pi\left(v_{\mathrm{L}}, \hat{v}_{\mathrm{L}}\right)^{2}} \Delta^{(\hat{2}, 2)} T^{(-2,-4)}\left(\hat{v}_{\mathrm{L}}, v_{\mathrm{R}}\right)
$$

where we have defined

$$
\Delta^{(\hat{2}, 2)}=\frac{\mathrm{i}}{4} \hat{v}_{i} \hat{v}_{j} v_{\bar{i}} v_{\bar{j}} \nabla^{\alpha i \bar{i}} \nabla_{\alpha}^{j \bar{j}}
$$

We now obtain an alternative representation for the left action (D.12). The idea is to insert the unity $1=G_{\mathrm{L}}^{(2)}\left(v_{\mathrm{L}}\right) / G_{\mathrm{L}}^{(2)}\left(v_{\mathrm{L}}\right)$ into the integrand (D.12), make use of the expression (D.22) for the field strength in the numerator and then integrate by parts in order to let $\Delta_{\mathrm{L}}^{(4)}$ hit $C_{\mathrm{L}}^{(-4)}$. This gives

$$
\begin{aligned}
S_{\mathrm{L}}= & \oint_{\gamma_{\mathrm{R}}} \frac{\left(v_{\mathrm{R}}, \mathrm{d} v_{\mathrm{R}}\right)}{2 \pi} \int \mathrm{d}^{3} x \mathrm{~d}^{8} \theta E \oint_{\gamma_{\mathrm{L}}} \frac{\left(v_{\mathrm{L}}, \mathrm{d} v_{\mathrm{L}}\right)}{2 \pi} \frac{\mathcal{L}_{\mathrm{L}}^{(2)}\left(v_{\mathrm{L}}\right)}{G_{\mathrm{L}}^{(2)}\left(v_{\mathrm{L}}\right)} \\
& \times \oint_{\hat{\gamma}_{\mathrm{L}}} \frac{\left(\hat{v}_{\mathrm{L}}, \mathrm{d} \hat{v}_{\mathrm{L}}\right)}{2 \pi\left(v_{\mathrm{L}}, \hat{v}_{\mathrm{L}}\right)^{2}} \Delta^{(\hat{2}, 2)} T^{(-2,-4)}\left(\hat{v}_{\mathrm{L}}, v_{\mathrm{R}}\right),
\end{aligned}
$$

\footnotetext{
${ }^{30}$ See [52] for the definition of $\mathcal{N}=4$ isotwistor superfields.

${ }^{31}$ For instance, we can choose $T^{(-2,-4)}\left(\hat{v}_{\mathrm{L}}, v_{\mathrm{R}}\right)=-2 T_{\mathrm{R}}^{(-4)}\left(v_{\mathrm{R}}\right) \frac{G_{\mathrm{L}}}{G_{\mathrm{L}}^{(2)}\left(\hat{v}_{\mathrm{L}}\right)}$.
} 
where we have changed the order of contour integrals. The next step is to insert the unity $\Delta_{\mathrm{R}}^{(4)} C_{\mathrm{R}}^{(-4)}=1$ into the integrand and then integrate by parts. This gives

$$
\begin{aligned}
S_{\mathrm{L}}= & \oint_{\gamma_{\mathrm{R}}} \frac{\left(v_{\mathrm{R}}, \mathrm{d} v_{\mathrm{R}}\right)}{2 \pi} \int \mathrm{d}^{3} x \mathrm{~d}^{8} \theta E C_{\mathrm{R}}^{(-4)} \\
& \times \Delta_{\mathrm{R}}^{(4)} \oint_{\gamma_{\mathrm{L}}} \frac{\left(v_{\mathrm{L}}, \mathrm{d} v_{\mathrm{L}}\right)}{2 \pi} \frac{\mathcal{L}_{\mathrm{L}}^{(2)}\left(v_{\mathrm{L}}\right)}{G_{\mathrm{L}}^{(2)}\left(v_{\mathrm{L}}\right)} \oint_{\hat{\gamma}_{\mathrm{L}}} \frac{\left(\hat{v}_{\mathrm{L}}, \mathrm{d} \hat{v}_{\mathrm{L}}\right)}{2 \pi\left(v_{\mathrm{L}}, \hat{v}_{\mathrm{L}}\right)^{2}} \Delta^{(\hat{2}, 2)} T^{(-2,-4)}\left(\hat{v}_{\mathrm{L}}, v_{\mathrm{R}}\right) .
\end{aligned}
$$

Taking the explicit form of $\Delta_{\mathrm{R}}^{(4)}$ into account, this expression can be replaced with the following:

$$
\begin{aligned}
S_{\mathrm{L}}= & -\oint_{\gamma_{\mathrm{R}}} \frac{\left(v_{\mathrm{R}}, \mathrm{d} v_{\mathrm{R}}\right)}{2 \pi} \int \mathrm{d}^{3} x \mathrm{~d}^{8} \theta E C_{\mathrm{R}}^{(-4)} \oint_{\gamma_{\mathrm{L}}} \frac{\left(v_{\mathrm{L}}, \mathrm{d} v_{\mathrm{L}}\right)}{2 \pi} \\
& \times \Delta^{(-2,2)}\left\{\frac{\mathcal{L}_{\mathrm{L}}^{(2)}\left(v_{\mathrm{L}}\right)}{G_{\mathrm{L}}^{(2)}\left(v_{\mathrm{L}}\right)} \oint_{\hat{\gamma}_{\mathrm{L}}} \frac{\left(\hat{v}_{\mathrm{L}}, \mathrm{d} \hat{v}_{\mathrm{L}}\right)}{2 \pi\left(v_{\mathrm{L}}, \hat{v}_{\mathrm{L}}\right)^{2}} \Delta^{(2,2)} \Delta^{(\hat{2}, 2)} T^{(-2,-4)}\left(\hat{v}_{\mathrm{L}}, v_{\mathrm{R}}\right)\right\} .
\end{aligned}
$$

Here the operator $\Delta^{(-2,2)}$ is defined by

$$
\Delta^{(-2,2)}:=\frac{u_{i} u_{j}}{\left(v_{\mathrm{L}}, u_{\mathrm{L}}\right)^{2}} v_{\bar{i}} v_{j}^{-} \nabla^{\alpha i \bar{i}} \nabla_{\alpha}^{j \bar{j}},
$$

for an isospinor $u_{i}$ such that $\left(v_{\mathrm{L}}, u_{\mathrm{L}}\right) \neq 0$. The operator $\Delta^{(2,2)}$ in (D.26) is obtained from $\Delta^{(\hat{2}, 2)}$, eq. (D.23), by replacing $\hat{v}_{i} \rightarrow v_{i}$. Now, one may notice that $\Delta^{(2,2)} \Delta^{(\hat{2}, 2)}$ in (D.26) is equivalent to $\left(v_{\mathrm{L}}, \hat{v}_{\mathrm{L}}\right)^{2} \Delta_{\mathrm{R}}^{(4)}$, and therefore the action turns into

$$
S_{\mathrm{L}}=\oint_{\gamma_{\mathrm{R}}} \frac{\left(v_{\mathrm{R}}, \mathrm{d} v_{\mathrm{R}}\right)}{2 \pi} \int \mathrm{d}^{3} x \mathrm{~d}^{8} \theta E C_{\mathrm{R}}^{(-4)} \oint_{\gamma_{\mathrm{L}}} \frac{\left(v_{\mathrm{L}}, \mathrm{d} v_{\mathrm{L}}\right)}{2 \pi} \Delta^{(-2,2)}\left\{\frac{\mathcal{L}_{\mathrm{L}}^{(2)}\left(v_{\mathrm{L}}\right)}{G_{\mathrm{L}}^{(2)}\left(v_{\mathrm{L}}\right)} \mathcal{V}_{\mathrm{R}}\left(v_{\mathrm{R}}\right)\right\} .
$$

Since $\mathcal{V}_{\mathrm{R}}$ is a right projective multiplet, it commutes with the operator $\Delta^{(-2,2)}$, and we end up with the following representation for $S_{\mathrm{L}}$ :

$$
S_{\mathrm{L}}=\oint_{\gamma_{\mathrm{R}}} \frac{\left(v_{\mathrm{R}}, \mathrm{d} v_{\mathrm{R}}\right)}{2 \pi} \int \mathrm{d}^{3} x \mathrm{~d}^{8} \theta E C_{\mathrm{R}}^{(-4)} \mathcal{V}_{\mathrm{R}} \boldsymbol{G}_{\mathrm{R}}^{(2)}
$$

where

$$
\boldsymbol{G}_{\mathrm{R}}^{(2)}\left(v_{\mathrm{R}}\right)=v_{\bar{i}} v_{\bar{j}} \boldsymbol{G}^{\bar{i} \bar{j}}=\frac{\mathrm{i}}{4} v_{\bar{i}} v_{\bar{j}} \oint \frac{\left(v_{\mathrm{L}}, \mathrm{d} v_{\mathrm{L}}\right)}{2 \pi} \frac{u_{i} u_{j}}{\left(v_{\mathrm{L}}, u_{\mathrm{L}}\right)^{2}} \nabla^{\alpha i \bar{i}} \nabla_{\alpha}^{j \bar{j}}\left\{\frac{\mathcal{L}_{\mathrm{L}}^{(2)}\left(v_{\mathrm{L}}\right)}{G_{\mathrm{L}}^{(2)}\left(v_{\mathrm{L}}\right)}\right\}
$$

is a composite right $\mathcal{O}(2)$ multiplet.

Eq. (D.29) is our new representation for the left action (D.11). The important point is that the integration in (D.11) and (D.29) is carried out over different subspaces of the curved projective superspace. The original left action (D.11) is given as an integral over $\mathcal{M}^{3 \mid 8} \times \mathbb{C} P_{\mathrm{L}}^{1}$. In the final action (D.29), the integration is carried out over $\mathcal{M}^{3 \mid 8} \times \mathbb{C} P_{\mathrm{R}}^{1}$.

Since (D.29) involves the composite right $\mathcal{O}(2)$ multiplet $G_{\mathrm{R}}^{(2)}$, it will be referred to as the right linear multiplet action. 
Open Access. This article is distributed under the terms of the Creative Commons Attribution License (CC-BY 4.0), which permits any use, distribution and reproduction in any medium, provided the original author(s) and source are credited.

\section{References}

[1] M.F. Sohnius, Supersymmetry and central charges, Nucl. Phys. B 138 (1978) 109 [INSPIRE].

[2] P. Fayet, Fermi-Bose hypersymmetry, Nucl. Phys. B 113 (1976) 135 [INSPIRE].

[3] R. Grimm, M. Sohnius and J. Wess, Extended supersymmetry and gauge theories, Nucl. Phys. B 133 (1978) 275 [InSPIRE].

[4] P. Breitenlohner and M.F. Sohnius, Superfields, auxiliary fields and tensor calculus for $N=2$ extended supergravity, Nucl. Phys. B 165 (1980) 483 [INSPIRE].

[5] B. de Wit, J.W. van Holten and A. Van Proeyen, Central charges and conformal supergravity, Phys. Lett. B 95 (1980) 51 [inSPIRE].

[6] B. de Wit, J.W. van Holten and A. Van Proeyen, Transformation rules of $N=2$ supergravity multiplets, Nucl. Phys. B 167 (1980) 186 [InSPIRE].

[7] E. Bergshoeff, M. de Roo and B. de Wit, Extended conformal supergravity, Nucl. Phys. B 182 (1981) 173 [InSPIRE].

[8] B. de Wit, J.W. van Holten and A. Van Proeyen, Structure of $N=2$ supergravity, Nucl. Phys. B 184 (1981) 77 [Erratum ibid. B 222 (1983) 516] [INSPIRE].

[9] D.Z. Freedman and A. Van Proeyen, Supergravity, Cambridge University Press, Cambridge U.K. (2012).

[10] M. Zucker, Gauged N=2 off-shell supergravity in five-dimensions, JHEP 08 (2000) 016 [hep-th/9909144] [INSPIRE].

[11] M. Zucker, Off-shell supergravity in five-dimensions and supersymmetric brane world scenarios, Fortsch. Phys. 51 (2003) 899 [INSPIRE].

[12] T. Kugo and K. Ohashi, Supergravity tensor calculus in $5-D$ from $6 D$, Prog. Theor. Phys. 104 (2000) 835 [hep-ph/0006231] [InSPIRE].

[13] T. Kugo and K. Ohashi, Off-shell $d=5$ supergravity coupled to matter Yang-Mills system, Prog. Theor. Phys. 105 (2001) 323 [hep-ph/0010288] [INSPIRE].

[14] T. Fujita and K. Ohashi, Superconformal tensor calculus in five-dimensions, Prog. Theor. Phys. 106 (2001) 221 [hep-th/0104130] [INSPIRE].

[15] E. Bergshoeff et al., Weyl multiplets of $N=2$ conformal supergravity in five-dimensions, JHEP 06 (2001) 051 [hep-th/0104113] [INSPIRE].

[16] E. Bergshoeff et al., Superconformal $N=2, D=5$ matter with and without actions, JHEP 10 (2002) 045 [hep-th/0205230] [INSPIRE].

[17] E. Bergshoeff et al., $N=2$ supergravity in five-dimensions revisited, Class. Quant. Grav. 21 (2004) 3015 [hep-th/0403045] [INSPIRE].

[18] E. Bergshoeff, E. Sezgin and A. Van Proeyen, Superconformal tensor calculus and matter couplings in six-dimensions, Nucl. Phys. B 264 (1986) 653 [Erratum ibid. B 598 (2001) 667] [INSPIRE].

[19] S.M. Kuzenko and G. Tartaglino-Mazzucchelli, Five-dimensional superfield supergravity, Phys. Lett. B 661 (2008) 42 [arXiv:0710.3440] [INSPIRE]. 
[20] S.M. Kuzenko and G. Tartaglino-Mazzucchelli, 5D supergravity and projective superspace, JHEP 02 (2008) 004 [arXiv:0712.3102] [INSPIRE].

[21] S.M. Kuzenko and G. Tartaglino-Mazzucchelli, Super-Weyl invariance in $5 D$ supergravity, JHEP 04 (2008) 032 [arXiv:0802.3953] [INSPIRE].

[22] M.F. Sohnius, K.S. Stelle and P.C. West, Representations of extended supersymmetry, in Superspace and Supergravity, S.W. Hawking and M. Roček eds., Cambridge University Press, Cambridge U.K. (1981), pg. 283.

[23] U. Lindström and M. Roček, Properties of hyperKähler manifolds and their twistor spaces, Commun. Math. Phys. 293 (2010) 257 [arXiv:0807.1366] [INSPIRE].

[24] S.M. Kuzenko, Lectures on nonlinear $\sigma$-models in projective superspace, J. Phys. A 43 (2010) 443001 [arXiv: 1004.0880] [inSPIRE].

[25] J. Wess, Supersymmetry and internal symmetry, Acta Phys. Austriaca 41 (1975) 409 [INSPIRE].

[26] S.M. Kuzenko, U. Lindström, M. Roček and G. Tartaglino-Mazzucchelli, $4 D N=2$ supergravity and projective superspace, JHEP 09 (2008) 051 [arXiv:0805.4683] [INSPIRE].

[27] S.M. Kuzenko, On $N=2$ supergravity and projective superspace: dual formulations, Nucl. Phys. B 810 (2009) 135 [arXiv:0807.3381] [INSPIRE].

[28] S.M. Kuzenko, U. Lindström, M. Roček and G. Tartaglino-Mazzucchelli, On conformal supergravity and projective superspace, JHEP 08 (2009) 023 [arXiv: 0905. 0063] [INSPIRE].

[29] S.M. Kuzenko and G. Tartaglino-Mazzucchelli, Different representations for the action principle in $4 D N=2$ supergravity, JHEP 04 (2009) 007 [arXiv:0812.3464] [INSPIRE].

[30] S.M. Kuzenko and S. Theisen, Correlation functions of conserved currents in $N=2$ superconformal theory, Class. Quant. Grav. 17 (2000) 665 [hep-th/9907107] [INSPIRE].

[31] N. Dragon, E. Ivanov, S. Kuzenko, E. Sokatchev and U. Theis, $N=2$ rigid supersymmetry with gauged central charge, Nucl. Phys. B 538 (1999) 411 [hep-th/9805152] [INSPIRE].

[32] I. Linch, William D. and G. Tartaglino-Mazzucchelli, Six-dimensional supergravity and projective superfields, JHEP 08 (2012) 075 [arXiv:1204.4195] [INSPIRE].

[33] A. Galperin, E. Ivanov, S. Kalitsyn, V. Ogievetsky and E. Sokatchev, Unconstrained $N=2$ Matter, Yang-Mills and supergravity theories in harmonic superspace, Class. Quant. Grav. 1 (1984) 469 [inSPIRE].

[34] A.S. Galperin, E.A. Ivanov, V.I. Ogievetsky and E.S. Sokatchev, Harmonic Superspace, Cambridge University Press, Cambridge U.K. (2001).

[35] A. Karlhede, U. Lindström and M. Roček, Selfinteracting tensor multiplets in $N=2$ superspace, Phys. Lett. B 147 (1984) 297 [INSPIRE].

[36] U. Lindström and M. Roček, New hyperkähler metrics and new supermultiplets, Commun. Math. Phys. 115 (1988) 21 [INSPIRE].

[37] U. Lindström and M. Roček, $N=2$ super Yang-Mills theory in projective superspace, Commun. Math. Phys. 128 (1990) 191 [inSPIRE].

[38] D. Butter, S.M. Kuzenko and J. Novak, The linear multiplet and ectoplasm, JHEP 09 (2012) 131 [arXiv: 1205.6981] [INSPIRE].

[39] D. Butter, $N=2$ conformal superspace in four dimensions, JHEP 10 (2011) 030 [arXiv:1103.5914] [INSPIRE]. 
[40] L. Castellani, R. D'Auria and P. Fre, Supergravity and superstrings: A Geometric perspective. Vol. 2: Supergravity, World Scientific, Singapore (1991), pg. 680.

[41] M.F. Hasler, The three form multiplet in $N=2$ superspace, Eur. Phys. J. C 1 (1998) 729 [hep-th/9606076] [INSPIRE].

[42] S.J. Gates Jr., Ectoplasm has no topology: the prelude, in Supersymmetries and Quantum Symmetries, J. Wess and E.A. Ivanov eds., Springer, Berlin Germany (1999), pg. 46 [hep-th/9709104] [INSPIRE].

[43] S.J. Gates Jr., M.T. Grisaru, M.E. Knutt-Wehlau and W. Siegel, Component actions from curved superspace: normal coordinates and ectoplasm, Phys. Lett. B 421 (1998) 203 [hep-th/9711151] [INSPIRE].

[44] S.M. Kuzenko and G. Tartaglino-Mazzucchelli, Conformal supergravities as Chern-Simons theories revisited, JHEP 03 (2013) 113 [arXiv: 1212.6852] [INSPIRE].

[45] D. Butter, S.M. Kuzenko, J. Novak and G. Tartaglino-Mazzucchelli, Conformal supergravity in three dimensions: off-shell actions, JHEP 10 (2013) 073 [arXiv: 1306.1205] [INSPIRE].

[46] S.M. Kuzenko, J. Novak and G. Tartaglino-Mazzucchelli, $N=6$ superconformal gravity in three dimensions from superspace, JHEP 01 (2014) 121 [arXiv:1308.5552] [INSPIRE].

[47] M. Nishimura and Y. Tanii, $N=6$ conformal supergravity in three dimensions, JHEP 10 (2013) 123 [arXiv: 1308.3960] [INSPIRE].

[48] P. van Nieuwenhuizen, $D=3$ conformal supergravity and Chern-Simons terms, Phys. Rev. D 32 (1985) 872 [INSPIRE].

[49] M. Roček and P. van Nieuwenhuizen, $N \geq 2$ supersymmetric Chern-Simons terms as $d=3$ extended conformal supergravity, Class. Quant. Grav. 3 (1986) 43 [INSPIRE].

[50] D. Butter, S.M. Kuzenko, J. Novak and G. Tartaglino-Mazzucchelli, Conformal supergravity in three dimensions: new off-shell formulation, JHEP 09 (2013) 072 [arXiv:1305.3132] [INSPIRE].

[51] P.S. Howe, J.M. Izquierdo, G. Papadopoulos and P.K. Townsend, New supergravities with central charges and Killing spinors in (2+1)-dimensions, Nucl. Phys. B 467 (1996) 183 [hep-th/9505032] [INSPIRE].

[52] S.M. Kuzenko, U. Lindström and G. Tartaglino-Mazzucchelli, Off-shell supergravity-matter couplings in three dimensions, JHEP 03 (2011) 120 [arXiv:1101.4013] [INSPIRE].

[53] W. Siegel, Unextended superfields in extended supersymmetry, Nucl. Phys. B 156 (1979) 135 [INSPIRE].

[54] S.J. Gates Jr., M.T. Grisaru, M. Roček and W. Siegel, Superspace or one thousand and one lessons in supersymmetry, Front. Phys. 58 (1983) 1 [hep-th/0108200] [INSPIRE].

[55] B.M. Zupnik and D.G. Pak, Superfield formulation of the simplest three-dimensional gauge theories and conformal supergravities, Theor. Math. Phys. 77 (1988) 1070 [INSPIRE].

[56] E.A. Ivanov, Chern-Simons matter systems with manifest $N=2$ supersymmetry, Phys. Lett. B 268 (1991) 203 [INSPIRE].

[57] B.M. Zupnik and D.V. Khetselius, Three-dimensional extended supersymmetry in the harmonic superspace. (In Russian), Sov. J. Nucl. Phys. 47 (1988) 730 [Yad. Fiz. 47 (1988) 1147] [INSPIRE].

[58] R. Brooks and S.J. Gates Jr., Extended supersymmetry and superBF gauge theories, Nucl. Phys. B 432 (1994) 205 [hep-th/9407147] [INSPIRE]. 
[59] A. Kapustin and M.J. Strassler, On mirror symmetry in three-dimensional Abelian gauge theories, JHEP 04 (1999) 021 [hep-th/9902033] [INSPIRE].

[60] B. Zupnik, Harmonic superpotentials and symmetries in gauge theories with eight supercharges, Nucl. Phys. B 554 (1999) 365 [Erratum ibid. B 644 (2002) 405] [hep-th/9902038] [INSPIRE].

[61] B.M. Zupnik, Three-dimensional $N=4$ superconformal superfield theories, Theor. Math. Phys. 162 (2010) 74 [arXiv:0905.1179] [INSPIRE].

[62] B.M. Zupnik, Three-dimensional $\mathcal{N}=4$ supersymmetry in harmonic $\mathcal{N}=3$ superspace, Theor. Math. Phys. 165 (2010) 1315 [arXiv:1005.4750] [InSPIRE].

[63] B. de Wit, A.K. Tollsten and H. Nicolai, Locally supersymmetric $D=3$ nonlinear $\sigma$-models, Nucl. Phys. B 392 (1993) 3 [hep-th/9208074] [INSPIRE].

[64] B. de Wit, I. Herger and H. Samtleben, Gauged locally supersymmetric D $=3$ nonlinear $\sigma$-models, Nucl. Phys. B 671 (2003) 175 [hep-th/0307006] [INSPIRE].

[65] B. de Wit, H. Nicolai and H. Samtleben, Gauged supergravities in three-dimensions: a panoramic overview, hep-th/0403014 [INSPIRE].

[66] E. Bergshoeff, S. Cecotti, H. Samtleben and E. Sezgin, Superconformal $\sigma$-models in three dimensions, Nucl. Phys. B 838 (2010) 266 [arXiv:1002.4411] [INSPIRE].

[67] H. Nishino and S. Rajpoot, Supersymmetric $E(8(+8)) / \mathrm{SO}(16) \sigma$-model coupled to $N=1$ supergravity in three-dimensions, Phys. Lett. B 535 (2002) 337 [hep-th/0203102] [INSPIRE].

[68] N.J. Hitchin, A. Karlhede, U. Lindström and M. Roček, HyperKähler metrics and supersymmetry, Commun. Math. Phys. 108 (1987) 535 [INSPIRE].

[69] M.F. Sohnius, Bianchi identities for supersymmetric gauge theories, Nucl. Phys. B 136 (1978) 461 [InSPIRE].

[70] U. Gran, J. Greitz, P.S. Howe and B.E.W. Nilsson, Topologically gauged superconformal Chern-Simons matter theories, JHEP 12 (2012) 046 [arXiv:1204.2521] [INSPIRE].

[71] L. Bonora, P. Pasti and M. Tonin, Chiral anomalies in higher dimensional supersymmetric theories, Nucl. Phys. B 286 (1987) 150 [InSPIRE].

[72] G. Bossard, P.S. Howe, U. Lindström, K.S. Stelle and L. Wulff, Integral invariants in maximally supersymmetric Yang-Mills theories, JHEP 05 (2011) 021 [arXiv:1012.3142] [INSPIRE].

[73] G. Bossard, P.S. Howe and K.S. Stelle, Invariants and divergences in half-maximal supergravity theories, JHEP 07 (2013) 117 [arXiv:1304.7753] [INSPIRE].

[74] J. Novak, Superform formulation for vector-tensor multiplets in conformal supergravity, JHEP 09 (2012) 060 [arXiv: 1205.6881] [INSPIRE].

[75] H. Nishino and S.J. Gates Jr., Chern-Simons theories with supersymmetries in three-dimensions, Int. J. Mod. Phys. A 8 (1993) 3371 [INSPIRE].

[76] J. Wess and B. Zumino, The component formalism follows from the superspace formulation of supergravity, Phys. Lett. B 79 (1978) 394 [INSPIRE].

[77] J. Wess and J. Bagger, Supersymmetry and Supergravity, Princeton University Press, Princeton U.S.A. (1992).

[78] S.M. Kuzenko and G. Tartaglino-Mazzucchelli, Three-dimensional $N=2$ (AdS) supergravity and associated supercurrents, JHEP 12 (2011) 052 [arXiv:1109.0496] [INSPIRE]. 
[79] S.M. Kuzenko, Prepotentials for $N=2$ conformal supergravity in three dimensions, JHEP 12 (2012) 021 [arXiv:1209.3894] [INSPIRE].

[80] S.M. Kuzenko, U. Lindström, M. Roček, I. Sachs and G. Tartaglino-Mazzucchelli, Three-dimensional $N=2$ supergravity theories: From superspace to components, Phys. Rev. D 89 (2014) 085028 [arXiv:1312.4267] [InSPIRE].

[81] D. Butter and J. Novak, Component reduction in $N=2$ supergravity: the vector, tensor and vector-tensor multiplets, JHEP 05 (2012) 115 [arXiv: 1201.5431] [INSPIRE].

[82] B. de Wit, R. Philippe and A. Van Proeyen, The improved tensor multiplet in $N=2$ supergravity, Nucl. Phys. B 219 (1983) 143 [InSPIRE].

[83] U. Lindström and M. Roček, Scalar tensor duality and $N=1, N=2$ nonlinear $\sigma$-models, Nucl. Phys. B 222 (1983) 285 [inSPIRE].

[84] B. de Wit and M. Roček, Improved tensor multiplets, Phys. Lett. B 109 (1982) 439 [INSPIRE].

[85] D. Butter and S.M. Kuzenko, New higher-derivative couplings in $4 D N=2$ supergravity, JHEP 03 (2011) 047 [arXiv: 1012.5153] [INSPIRE].

[86] A. Achucarro and P.K. Townsend, A Chern-Simons action for three-dimensional anti-de Sitter supergravity theories, Phys. Lett. B 180 (1986) 89 [INSPIRE].

[87] S.M. Kuzenko, U. Lindström and G. Tartaglino-Mazzucchelli, Three-dimensional ( $p, q)$ AdS superspaces and matter couplings, JHEP 08 (2012) 024 [arXiv: 1205.4622] [INSPIRE].

[88] S. Deser and J.H. Kay, Topologically massive supergravity, Phys. Lett. B 120 (1983) 97 [INSPIRE].

[89] S. Deser, Cosmological topological supergravity, in Quantum Theory Of Gravity, S.M. Christensen eds., Adam Hilger, Bristol U.K. (1984) pg. 374.

[90] I.L. Buchbinder, S.M. Kuzenko and A.A. Tseytlin, On low-energy effective actions in $N=2$, $N=4$ superconformal theories in four-dimensions, Phys. Rev. D 62 (2000) 045001 [hep-th/9911221] [INSPIRE].

[91] B. de Wit, S. Katmadas and M. van Zalk, New supersymmetric higher-derivative couplings: full $N=2$ superspace does not count!, JHEP 01 (2011) 007 [arXiv:1010.2150] [INSPIRE].

[92] S. Katmadas and R. Minasian, $\mathcal{N}=2$ higher-derivative couplings from strings, JHEP 02 (2014) 093 [arXiv: 1311.4797] [INSPIRE].

[93] P.S. Howe and M.I. Leeming, Harmonic superspaces in low dimensions, Class. Quant. Grav. 11 (1994) 2843 [hep-th/9408062] [INSPIRE].

[94] B.M. Zupnik, Chern-Simons $D=3, N=6$ superfield theory, Phys. Lett. B 660 (2008) 254 [arXiv:0711.4680] [INSPIRE].

[95] B.M. Zupnik, Chern-Simons theory in $\mathrm{SO}(5) / \mathrm{U}(2)$ harmonic superspace, Theor. Math. Phys. 157 (2008) 1550 [arXiv:0802.0801] [INSPIRE].

[96] I.L. Buchbinder et al., Quantum $N=3, D=3$ Chern-Simons matter theories in harmonic superspace, JHEP 10 (2009) 075 [arXiv: 0909.2970] [INSPIRE]. 